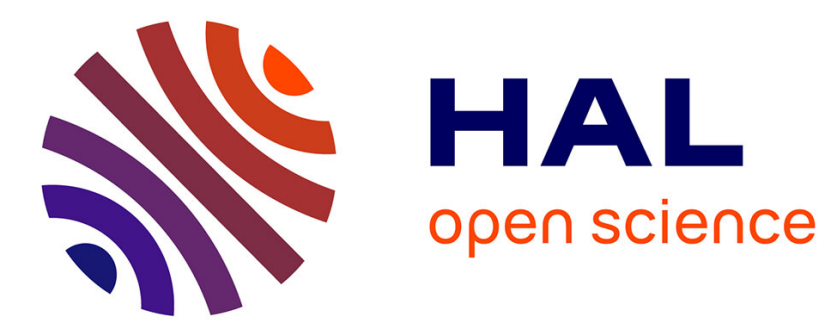

\title{
Adding a new separation dimension to MS and LC-MS: What is the utility of ion mobility spectrometry?
} \author{
Valentina d'Atri, Tim Causon, Oscar Hernandez-Alba, Aline Mutabazi,
} Jean-Luc Veuthey, Sarah Cianférani, Davy Guillarme

\section{To cite this version:}

Valentina d'Atri, Tim Causon, Oscar Hernandez-Alba, Aline Mutabazi, Jean-Luc Veuthey, et al.. Adding a new separation dimension to MS and LC-MS: What is the utility of ion mobility spectrometry?. Journal of Separation Science, 2018, 41 (1), pp.20 - 67. 10.1002/jssc.201700919 . hal-01702282

\section{HAL Id: hal-01702282 \\ https://hal.science/hal-01702282}

Submitted on 8 Oct 2020

HAL is a multi-disciplinary open access archive for the deposit and dissemination of scientific research documents, whether they are published or not. The documents may come from teaching and research institutions in France or abroad, or from public or private research centers.
L'archive ouverte pluridisciplinaire HAL, est destinée au dépôt et à la diffusion de documents scientifiques de niveau recherche, publiés ou non, émanant des établissements d'enseignement et de recherche français ou étrangers, des laboratoires publics ou privés. 
Adding a new separation dimension to MS and LC-MS: what is the utility of ion mobility spectrometry?

3

4 AUTHORS: Valentina D'ATRI ${ }^{(1) \ddagger}$, Tim CAUSON ${ }^{(2) \ddagger}$, Oscar HERNANDEZ-ALBA ${ }^{(3) \ddagger}$, Aline 5 MUTABAZI $^{(1) \ddagger}$, Jean-Luc VEUTHEY(1), Sarah CIANFERANI(3), Davy GUILLARME $^{(1)}$

7 (1) School of Pharmaceutical Sciences, University of Geneva, University of Lausanne, CMU 8 Rue Michel Servet 1, 1211 Geneva 4, Switzerland

(2) Division of Analytical Chemistry, Department of Chemistry, University of Natural Resources

10 and Life Sciences (BOKU Vienna), Muthgasse 18, Vienna 1190, Austria

11 (3) BioOrganic Mass Spectrometry Laboratory (LSMBO), IPHC, Université de Strasbourg, 12 CNRS, IPHC UMR 7178, 67000 Strasbourg, France

RUNNING TITLE: IM-MS and its applications

17 CORRESPONDENCE: Davy GUILLARME

18 School of Pharmaceutical Sciences, University of Geneva, University of Lausanne, CMU - Rue 19 Michel Servet 1, 1211 Geneva 4, Switzerland

20 Phone: +41223793463

$21 \quad$ E-mail: davy.guillarme@unige.ch

22

23

24 KEYWORDS: collision cross section values; ion mobility spectrometry; metabolomics; 25 biopharmaceuticals, oligonucleotides

26

$27 \quad(\ddagger)$ These authors contributed equally 


\section{LIST OF ABBREVIATIONS}

29 2D-LC: two-dimensional liquid chromatography

30 AD: alzheimer disease

31 ADC: antibody drug conjugate

32 AmtB: ammonia channel

33 APCl: atmospheric pressure chemical ionization

34 APols: amphipatic polymers

35 AqpZ: aquaporin Z

36 A.T: adenine thymine

37 ATD: arrival time distribution

38 bsAb: bispecific antibodies

39 BSM: bovine sumaxillary mucin

40 CCS: collisional cross section

41 CDL: cardiolipin

42 C.G: cytosine-guanine

43 CID: collision induced dissociation

44 CIU: collision induced unfolding

45 CRISP : clustered regularly interspaced short palindromic

46 CRISPR : clustered regularly interspaced short palindromic repeats

47 CZE: capillary zone electrophoresis

48 DAR: drug-to-antibody-ratio

49 DBD: DNA-binding domain

50 DC: differential current

51 DDA: data-dependent acquisition

52 DESI: desorption electrospray ionisation

53 DFT: density functional theory

54 DHSS: diffuse hard spheres scattering

55 DIA: data-independent acquisition 
56 DIMS: differential ion mobility spectrometry

57 DMA: differential mobility analyser

58 DMS: differential mobility spectrometry

59 DNA: deoxyribonucleic acid

$60{ }^{\mathrm{DT}} \mathrm{CCS}_{\mathrm{He}}$ : collisional cross section calculated with drift tube ion mobility spectrometry and

61 helium as buffer gas

$62{ }^{\mathrm{DT}} \mathrm{CCS}_{\mathrm{N} 2}$ : collisional cross section calculated with drift tube ion mobility spectrometry and

63 nitrogen as buffer gas

64 DTIMS: drift tube ion mobility spectrometry

65 DTM: diffuse trajectory method

66 EED: electron excitation dissociation

67 EHSS: exact hard sphere scattering

68 ESI: electrospray ionization

69 ETD: electron-transfer dissociation

70 FAIMS : field asymmetric waveform ion mobility spectrometry

71 FDA: food and drug administration

72 FID: flame ionization detector

73 FRET: fluorescence resonance energy transfer

74 GC: gas chromatography

75 HCP: host cell proteins

76 HD-DDA: high definition data direct analysis

77 HDX-MS: hydrogen/deuterium exchange mass spectrometry

78 HIC: hydrophobic interaction chromatography

79 HMGA: high-mobility group A

80 HRMS: high resolution mass spectrometry

81 IAPP: islet amyloid polypeptide

82 IDP: intrinsically disordered proteins

83 lgG: immunoglobulin G 
84 IM: ion mobility

85 IM-MS: ion mobility - mass spectrometry

86 IMS: ion mobility spectrometry

87 IMS-QTOF: ion mobility spectrometry quadrupole time-of-flight

88 IR: infrared radiation

89 IRMPD: infrared multiphoton dissociation

90 LC: liquid chromatography

91 LC-IMS-MS: liquid chromatography - ion mobility - mass spectrometry

92 LC-MS: liquid chromatography-mass spectrometry

93 LCPA: local collision probability approximation

94 LOD: limit of detection

95 mAb: monoclonal antibody

96 MALDI: matrix-assisted laser desorption ionisation

97 MC: Monte Carlo

98 MD: molecular dynamics

99 MS: mass spectrometry

100 MS/MS: tandem mass spectrometry

101 MscL: mechanosensitive channel of large conductance

102 NMR: nuclear magnetic resonance

103 PA: projection approximation

104 PASEF: parallel accumulation-serial fragmentation

105 PDB: protein data bank

106 PG: phosphatidylglycerol

107 PSA: projection superimposition approximation

108 PTM: posttranslational modifications

109 QM: quantum mechanical

110 QqTOF: quadrupole-time-of-flight

111 QTRAP: triple-quadrupole linear ion trap mass spectrometer 
112 RF: radio frequency

$113 \mathrm{rhGH}$ : recombinant human growth hormone

114 RNA: ribonucleic acid

115 SASA: solvent-accessible surface area

116 SEC: size exclusion chromatography

117 SFC: supercritical fluid chromatography

118 SID: surface induced dissociation

119 SLIM: structures for lossless ion manipulations

120 sno: small nucleolar

121 SRIG: stacked-ring ion guide

122 sRNPs: small nucleolar ribonucleoprotein particles

$123 \mathrm{~T}_{10}$ : thymine 10

124 T2DM: type 2 diabetes mellitus

125 Td: Townsends unit

126 TIMS: trapped ion mobility spectrometry

127 TIMS-MS: trapped ion mobility spectrometry - mass spectrometry

$128{ }^{\mathrm{TIM}} \mathrm{CCS}_{\mathrm{N} 2}$ : collisional cross section calculated with trapped ion mobility spectrometry and

129 nitrogen as buffer gas

130 TM: trajectory method

131 TOF: time-of-flight

132 TRAP: trp RNA binding attenuation protein

133 TTR: tetrameric transthyretin

$134{ }^{\mathrm{TW}} \mathrm{CCS}_{\mathrm{N} 2}$ : collisional cross section calculated with travelling wave ion mobility spectrometry

135 and nitrogen as buffer gas

136 TWIMS: travelling wave ion mobility spectrometry

137 VUVPD: vacuum ultraviolet photo dissociation

138 VT-IM-MS: variable temperature ion mobility-mass spectrometry

139 wt: wild type 
140 XUV: extreme ultraviolet light 


\section{Abstract}

142 Ion mobility spectrometry is an analytical technique known for more than 100 years, which

143 entails separating ions in the gas phase based on their size, shape and charge. While IMS

144 alone can be useful for some applications (mostly security analysis for detecting certain 145 classes of narcotics and explosives), it becomes even more powerful in combination with MS

146 and HPLC. Indeed, the limited resolving power of ion mobility spectrometry alone can be 147 tackled when combining this analytical strategy with MS or LC-MS.

148 Over the last few years, the hyphenation of ion mobility spectrometry to MS or LC-MS attracted 149 more and more interest, with significant progresses in both technical advances and pioneering 150 applications. This review describes the theoretical background, available technologies and 151 future capabilities of these techniques. It also highlights a wide range of applications, from 152 small molecules (natural products, metabolites, glycans, lipids) to large biomolecules (proteins, 153 protein complexes, biopharmaceuticals, oligonucleotides). 


\section{Introduction}

156 During the last decade, ion mobility spectrometry (IMS) has been applied in several fields, 157 starting from the analysis of chemical warfare agents, explosives and illicit drugs to the analysis 158 of biological samples [1-4]. Nowadays, the increased complexity of the samples (e.g. natural 159 products analysis, biopharmaceutical characterization, -omic samples such as proteins mixture 160 in proteomics or biofluids in metabolomics or lipidomics, etc) demands for analytical methods 161 able to offer very high resolving power to achieve analytical goals and acquire sufficient 162 information from the sample.

163 The addition of IMS dimension to the existing analytical setup can represent an attractive strategy to achieve this goal. [4] Indeed, IMS is a gas-phase separation technique that allows the separation of ions generated by a source (frequently by electrospray ionization, ESI) driven through an ion mobility cell filled with an inert gas (generally nitrogen or helium) under the

167 influence of an electric field. Once in the IM drift cell, ions are separated based on their mobility 168 that accounts for their size, shape and charge. Then, based on the type of IMS separator, the 169 mobility can also allow the determination of the collisional cross section (CCS), a physical 170 property related to the shape of the ion for a given compound in a specific gas environment.

171 The CCS is an important property that could provide an improved characterization of different compounds, such as small molecules, proteins, lipids or glycans, as well as a better 173 understanding of the intramolecular forces [5,6]. A detailed discussion about CCS 174 determination will be addressed in section 2.

175 As illustrated in Figure 1, IMS combined with mass spectrometry (MS), which is also known as 176 IMSxMS or IM-MS, has seen a spectacular growth in the last 20 years. Indeed, the information 177 obtained from IMS and MS can be considered as complementary and IMS can potentially allow 178 the separation of isobaric ions (unresolved by MS) based on their shape differences [7]. 179 Besides IMS and MS, a third separation dimension, such as liquid chromatography (LC) or 180 another front-end separation technique such as supercritical fluid chromatography (SFC), gas 181 chromatography (GC), or capillary zone electrophoresis (CZE) [8] can also be included in the 182 analytical setup. As shown in Figure 1, this three-dimensional separation (LC-IM-MS) is still 
183 less widespread than IMS alone or IM-MS, but is beginning to attract increasing interest for the 184 analyses of complex samples. As highlighted in Figure 2, LC-IM-MS allows the separation of 185 the different constituents of a mixture based on their lipophilicity (LC), shape (IMS), mass (MS) 186 and charge (IMS and MS). It also offers the possibility to visualize the analytical results in 187 several dimensions, using a plot of MS spectra information vs. IM drift times, for each 188 chromatographic peak.

189 Another important aspect is the time scale of IMS (in the milliseconds range), which fits very 190 well between the chromatographic step (in the minutes range) and MS experiment (in the 191 microseconds time scale). Because of the fast duty cycle of time-of-flight based mass spectrometer (TOF and QqTOF), these instruments appear to be the most suitable analysers for IM-MS [6].

194 This review paper describes the theoretical background of IM-MS technology, available 195 instrumentation and future capabilities of IM-MS. It also highlights a wide range of recent applications of IM-MS and LC-IM-MS, ranging from small molecules to large biomolecules.

\section{Description of the available ion mobility technologies}

Several ion mobility technologies have been developed in the last decades, each having its own principle of operation and design. According to May and McLean [9], there are three main separation concepts employed in IMS, namely i) time-dispersive methods, which generate an

202 arrival time spectrum, with all ions drifting along the same pathway (i.e. drift tube ion mobility 203 spectrometry (DTIMS), travelling wave ion mobility spectrometry (TWIMS)), ii) space204 dispersive methods, which separate ions along different drift paths, based on their mobility 205 differences (i.e. field asymmetric waveform ion mobility spectrometry (FAIMS), differential mobility analyser (DMA)) and iii) ion trapping followed by selective release, which traps ions within a pressurized region and selectively ejects the ions based on their mobility differences 208 (i.e. trapped ion mobility spectrometry (TIMS)). All the IMS techniques described in this section 209 can be coupled with MS [7], but performance and possibilities may vary, particularly in terms 210 of achievable limits of detection (LOD), resolving power (peak capacity) and possibility to 
211 accurately calculate CCS values. The main advantages, limitations and a list of available

212 commercial instruments for the different IMS technologies are described in this section and

213 summarized in Table 1. Last but not least, it is important to keep in mind that DTIMS and

214 TWIMS are the main choice for proteins, but have also been successfully used for small 215 molecules. On the other hand, FAIMS, DMA are only dedicated to the analysis of small 216 molecules. TIMS is a relatively new strategy, which also has the potential to be used for both 217 proteins and small molecules. However, it has only been scarcely employed for proteins and 218 more investigations are needed to prove its applicability for large biomolecules.

2.1. Drift tube ion mobility spectrometry

221 Drift tube ion mobility (DTIMS) is the historical and simplest form of IMS that was assembled 222 for the first time in the 1970s, originally in combination with a single quadrupole mass analyser 223 [10]. The schematic design of DTIMS is shown in Figure 3a. This technology is composed of 224 a tube (the drift cell) filled with an inert gas, namely the buffer gas. [2] Nitrogen or helium are 225 the most common buffer gas in DTIMS [11]. The DTIMS cell is composed of a series of piled electrodes, and each electrode generates a weak uniform electric field (5-100 V) [2] able to induce the movement of the ions through the tube. For example, on the Agilent DTIMS-MS instrument, $1000-1400 \mathrm{~V}$ is typically used in a $78 \mathrm{~cm}$ drift cell, corresponding to a field strength of around $13-18 \mathrm{~V} / \mathrm{cm}$. The mobility of ions along the cell is determined by the number of 230 collisions with the buffer gas, which slows down the ions: For a given charge, compact ions 231 drift faster than elongated ones, because they experience fewer collisions with the buffer gas. 232 [5] The time required to cross the cell is defined as the drift time and the separation of the ions occurs as a function of their shape, which is physically related to the mobility $(K)$ or rotationally averaged CCS value. However, the electric field has to be maintained sufficiently low to 235 observe a good correlation between mobility and shape/size.[7] As ions exit the tube, they are 236 analysed and detected as a function of time, yielding an arrival time distribution (ATD). The 237 first home-made DTIMS generally suffered from an inherent lack of sensitivity, mainly due to 238 diffusion issues and loss in duty cycle that was related to the pulsed analysis. However, in 
2014 a commercial DTIMS, using nitrogen drift gas and offering a good compromise between

240 sensitivity, length of drift tube, and optics, was developed by Agilent Technologies. In

241 particular, the instrument incorporates a tandem ion funnel interface before the drift tube that

242 acts as a focusing and accumulation region, and a second ion funnel after the drift tube that

243 refocuses the ions through an hexapole ion guide prior to reaching the QqTOFMS. The Agilent

244 DTIMS-MS instrument includes a QqTOF mass analyser, offers a high precision of CCS values

245 (below 0.5\% [12]), a high resolving power (thanks to the length of the tube extended to $78 \mathrm{~cm}$

246 combined with the precision electronics) and sensitivity compatible for protein/peptide

247 analyses [11,13]. Another DTIMS instrument commercially available is manufactured by

248 TOFWerk (Switzerland). This instrument contains a $20 \mathrm{~cm}$ drift cell operated at high pressure

249 (i.e. 1-1.4 atm) and elevated temperature (e.g. $\left.60^{\circ} \mathrm{C}\right)$, which provides greater resolution, while

250 maintaining low-field conditions, but is limited to low molecular weight molecules.

2.2. Travelling wave ion mobility spectrometry

Traveling wave ion mobility (TWIMS) is a more recent IMS technology that was first described in 2004 [14] and made commercially available in 2006 (Waters Synapt HDMS systems) [15]. This high performance instrument has significantly advanced the field of IM-MS thanks to its versatility and widespread availability [16]. Similarly to DTIMS, TWIMS is a time-dispersive

257 method, even though it uses a dynamic application of the electric field to separate the ions in 258 gaseous phase (often nitrogen and occasionally helium). It consists of a stacked-ring ion guide 259 (SRIG) where positive and negative radio frequency (RF) voltages are periodically applied to adjacent ring electrodes to provide radial ion confinement while a pulsed differential current 261 (DC) voltage is applied to axially propel the ions [2]. Therefore, rather than applying a linear electric field through the full length of the mobility cell, like in DTIMS, the electric field in TWIMS

263 forms a wave whose magnitude and speed determine the ions separation, as illustrated in

264 Figure 3b. By surfing on the waves, larger ions experience larger friction with the buffer gas 265 and slip more often behind the waves, thus taking more time to exit the mobility cell as compared to smaller ions. In TWIMS, CCS can also be determined from the drift time, but only 
267 after calibration by using a set of reference compounds of known CCS values. In addition, the

268 TWIMS technology takes less space than DTIMS (DTIMS needs around 1 meter to be installed

269 in front of the mass spectrometer) and is more versatile, since it allows to perform CID before 270 and after lon mobility (CID could only be performed after ion mobility with DTIMS) on the 271 commercial instruments (this is a choice of the constructors rather than a theoretical limitation).

272 As described in [9], the original Waters TWIMS technology underwent a major design 273 modification in 2009 (Synapt G2), allowing 4-fold improvement of the accessible resolution, 274 thanks to the use of higher electric fields and higher gas pressure [17]. In 2011 and 2013, two 275 new instruments were released by Waters, namely Synapt G2-S and G2-Si, allowing an 276 improvement of the ion transmission through the IM-MS and a wide range of experimental 277 options [9]. Finally, Waters has released a benchtop instrument in 2016 (Vion IMS QToF), 278 which includes in part the technology of the Synapt, but with a different design (the mobility 279 cell is located before the first quadrupole of the QqTOF/MS). Indeed, this instrument is 280 dedicated to non-experts who want to add an IMS dimension (determination of CCS values, 281 removal of spectral interferences) to routine analyses often performed in MS or LC-MS.

2.3. Field asymmetric waveform ion mobility spectrometry

284 Field asymmetric waveform ion mobility spectrometry (FAIMS) is also known as differential ion 285 mobility spectrometry (DMS or DIMS). The principle of operation of FAIMS is illustrated in 286 Figure 3c [18]. FAIMS utilizes two electrodes between which a high asymmetric electric field 287 (up to $10000 \mathrm{~V} / \mathrm{cm}$, also called dispersion field) is applied at reduced pressure (but can also 288 operate at atmospheric pressure) [19,20] and room temperature. The carrier gas (often 289 nitrogen, but there is a lot of flexibility to use some alternative gas or even dopants) is injected 290 with the ions in the same direction. A compensation voltage is then superimposed to the 291 dispersion field and it can be tuned for ions with a particular mobility [21]. By applying a suitable 292 compensation voltage, the trajectory of the desired ions can be corrected along the axis of the 293 cell to permit these ions to leave the cell. All other ions will migrate away from the straight line 
294 due to their different mobility. A full spectrum can finally be collected by scanning the 295 compensation voltage.

296 Unlike DTIMS and TWIMS, FAIMS does not separate ions based directly on their mobility, but 297 rather according to the ratio of mobilities in a low-field and high-field. Therefore, the FAIMS 298 separation is much more orthogonal to $\mathrm{m} / \mathrm{z}$ than other IMS modes (see section 8.2 for more 299 explanations). However, FAIMS is actually considered to be a filtering technique (or selective 300 scanning technique) rather than structure characterization tool, since calculation of CCS is not 301 possible [2]. Very high resolutions of up to several hundreds were reported in FAIMS, but this 302 was only possible when a mixture of gases was employed $\left(\mathrm{He} / \mathrm{N}_{2}\right)$ [22]. In addition, FAIMS 303 resolution cannot be directly compared to that of any other IMS technique described in this 304 review, since the separation phenomenon is different as is the scaling of the axes [16].

305 Several companies have commercialized FAIMS devices, including Thermo Fisher Scientific,

306 AB SCIEX and Owlstone. However, the most active provider is certainly SCIEX which utilises 307 FAIMS technology (SelexION) in several MS platforms, including QTRAP (Sciex 5500, 6500 308 and 6500+ series) and QqTOFs (Sciex TripleTOF 5600+ and 6600) analysers. On Thermo 309 Fisher Scientific instruments, the FAIMS interface can be added to existing triple quadrupole, 310 ion trap MS analysers and high-resolution orbital ion trap mass analysers [23].

313 Differential mobility analyser (DMA) is another form of IMS where the ions with different 314 mobilities are separated in space (rather than in time) at atmospheric pressure and relatively 315 low electric field. Two cylindrical and concentric metal electrodes constitute the usual DMA 316 design. A planar scheme of the DMA design is shown in the Figure 3d [24]. As illustrated in 317 this figure, the ions are injected along an electrical field (directed from top to bottom of the 318 scheme) between the two electrodes. An orthogonal flow of sheath gas from the left to the right 319 of the scheme pushes ions down from an inlet slit to the exit slit. Then, the ions whose mobility 320 matches the length from inlet slit to exit slit, at the used buffer gas flow and the electric field, 321 will reach the exit slit and will be directed towards the MS analyser [25]. 
322 The other ions, which do not match this mobility, will be eliminated on the lens that acts as a

323 wall. Only the ions with the targeted mobility will leave the DMA and the variation of the electric

324 field permits to select ions with different mobilities. Similarly to FAIMS, DMA is also considered

325 as a selective filter, but operates in the low-field regime. An important limitation of DMA is that

326 it does not allow the transmission of ions having diverse mobilities. The commercial availability

327 of DMA technology is relatively limited, and only a company called SEADM sells DMA systems

328 that can be directly coupled to MS.

2.5. Trapped ion mobility spectrometry

331 Trapped ion mobility spectrometry (TIMS) is a technology that appeared very recently on the market, which is based on the use of a non-uniform electric field [16]. In contrast to DTIMS or TWIMS, TIMS uses a high gas flow (exclusively nitrogen) to carry the ions of interest into the

334 drift cell. A low electric field is applied in the meantime in the opposite direction with the aim of 335 trapping and separating the ions as a function of their size-to-charge ratio. Then the electric 336 field is gradually decreased allowing the release of the ions from high (large CCS) to small 337 (small CCS) size-to-charge ratios [5]. A schematic of the separation process is shown in Figure $3 e$.

339 The TIMS-MS approach is still in early development and commercial devices have only been 340 released recently by Bruker (timsTOF) [26].

342 The advantages and limitations of these different IM-MS techniques above described are 343 summarized in Table 1.

\section{The importance of collision cross section}

3.1. Basic collision cross section equation

347 Ion mobility is a separation technique that allows analytes to be identified according to their 348 mass, charge and shape. The principle of IMS is often compared to that of electrophoresis, 349 even though the separation is conducted in the gas phase within a millisecond timescale. 
350 Considering a pool of gas-phase ions moving in a chamber filled with a buffer gas and under

351 the influence of an electric field $(E)$, the mobility $(K)$ is defined as the proportionality coefficient 352 between the strength of the electric field $E$ and the velocity $v_{d}$ attained by the ions:

353

$$
v_{d}=K \cdot E
$$

354

$355 K$ is usually reported as the reduced mobility $\left(K_{0}\right)$, upon normalization to standard pressure 356 and temperature $\left(p_{0}=760\right.$ Torr and $\left.T_{0}=273.15 \mathrm{~K}\right)$ :

$$
K_{0}=K \cdot \frac{p}{p_{0}} \cdot \frac{T_{0}}{T}
$$

In addition, $K_{0}$ can be related to the collision system parameters, accounting for the analyte characteristics under the experimental conditions, by the Mason-Schamp modification of the fundamental zero-field equation [27]:

361

$$
K_{0}=\frac{3 q}{16 N_{0}} \cdot\left(\frac{2 \pi}{k_{B} T}\right)^{\frac{1}{2}} \cdot\left(\frac{1}{m}+\frac{1}{M}\right)^{\frac{1}{2}} \cdot \frac{1}{\Omega}
$$

where $q=z e$ is the ionic charge ( $z$ is the nominal ion charge and $e$ is the elementary charge), $N_{0}$ is the gas density number at $p_{0}$ and $T_{0}, k_{B}$ is the Boltzmann's constant, $T$ is the gas temperature, $m$ is the mass of the buffer gas, while $M$ is the one of the ion, and $\Omega$ is the collision cross section (CCS).

367 While $q$ and $M$ take into account the charge and the mass of the ion, respectively, CCS $(\Omega)$ is

368 the physical quantity taking into account its "size and shape". CCS represents indeed the 369 effective area of the ion colliding with the molecules of the buffer gas. The higher the CCS, the 370 higher will be the number of collisions and thus more pronounced the effects on the ions that 371 will be slowed down by friction upon collisions. The CCS is thus a distinctive characteristic of each ion (this is true for small molecules) and it is considered as an important structural 
373 descriptor obtainable by IMS. However, it is important to keep in mind that the case of proteins

374 is more complex, since the activation conditions can influence the CCS and different CCS of 375 the same ion can co-exist. CCS can also be compared with other structural data obtained with 376 different analytical techniques, such as nuclear magnetic resonance (NMR), small-angle X-ray 377 scattering (SAXS), electron microscopy and X-ray crystallography. This point will be further 378 discussed in section 3.3. It is worth noticing that, according to Equations $1-3$, the mobility $(K)$ 379 depends on the density number of the drift gas $(N)$, and the proportional linearity between $v_{d}$ 380 and $E$ is only valid in the low electric field limit ( $E / N$ ratio $\leq 2$ Townsends $(T d))$. As the electric 381 field is increasing, $K$ is no longer constant and becomes field-dependent. Thus, the simple 382 relationship between $K_{0}$ and $1 / \Omega$ breaks down. This point is particularly important to understand 383 the limits of the experimental CCS determination and how to properly deduce it from measured 384 drift times on different types of IMS.

385

3.2. Experimental collision cross section determination

387 As described in section 1, several types of ion mobility separators can be coupled to MS. All 388 of these configurations allow the separation of the analytes based on their conformations, but 389 only a few of them can be used to determine CCS values. In this section, our goal was to 390 critically consider whether the IMS hardware allows the determination of experimental CCS. In 391 general, if the analyser operates in the low field limit $(E / N$ ratio $<2 \mathrm{Td})$ at all times and if the 392 field applied is constant, CCS might be directly determined from measured drift times (like in 393 DTIMS). If the IMS analyser operates in the low field limit, but with non-constant field due to 394 the application of additional radio frequencies (RF) or potentials, then a calibration with ions of 395 known CCS is required (like in TWIMS, DMA and TIMS). Finally, CCS cannot be derived at all 396 from the mobility data if the analyser operates at high field (like in FAIMS). The first two 397 scenarios allowing experimental CCS determination are discussed in further detail. 
(i) Low field limit at all times and application of constant field. The best example of this IMS

400 configuration is the DTIMS. Knowing the drift time ( $t_{d}$, time spent by the ions in the drift tube)

401 and that $v_{d}=L / t_{d}$ (where $L$ is the drift tube length), Equation 2 can be expressed as follow:

402

$$
K_{0}=\frac{L}{t_{d} E} \cdot \frac{p}{p_{0}} \cdot \frac{T_{0}}{T}
$$

403

404 with consequent CCS determination obtainable by $K_{0}$ following Equation 3 . Theoretically, 405 DTIMS analysers offer the most accurate CCS measurement using a stepped-field approach 406 without the need for calibration, assuming that all the parameters involved are accurately 407 known and carefully controlled. If not, a calibration with ions of known CCS could be necessary 408 also in this case. For online analysis (e.g. LC-IM-MS), a single-field calibration with ions of 409 known CCS is used to provide a regression model for converting drift time and mass into CCS. 410 In addition, the drift tube can operate with a wide range of neutral gases (e.g. nitrogen, helium, 411 carbon dioxide), thus CCS values are a conditional property. In this regard, users are beginning 412 to ensure appropriate reporting of CCS values with specific reference to the gas and type of 413 IM used (e.g. ${ }^{\mathrm{DT}} \mathrm{CCS}_{\mathrm{He}}$ or $\left.{ }^{\mathrm{DT}} \mathrm{CCS}_{\mathrm{N} 2}\right)$.

414 An important inter-laboratory study has been recently published [12] to highlight the trueness 415 and reproducibility of CCS measurement with DTIMS. In this work, 120 unique ion species (i.e. 416 metabolites, peptides, lipids and proteins, representing a wide range of masses, chemical 417 compositions and structures) were monitored in both positive and negative ionization modes, 418 and the reproducibility of ${ }^{\mathrm{D} T} \mathrm{CCS}_{\mathrm{N} 2}$ values were evaluated across three laboratories equipped 419 with commercial DTIMS-MS instruments. It appears that the average RSD on CCS values was 420 equal to only $0.29 \%$ for all ion species across the three laboratories equipped with the same 421 type of instrument. The trueness of the values, obtained with the commercial DTIMS-MS 422 instruments, was comprised between $0.34 \%$ and $0.54 \%$, depending whether a calibration was 423 applied or not. These results clearly illustrate the potential of DTIMS-MS for providing a 
424 molecular identifier for a broad range of applications, including untargeted screening 425 workflows.

427 (ii) Low field limit with non-constant field. The best examples for this IMS configuration are 428 TWIMS, DMA and TIMS. Due to the non-linearity of the electric field that complicates the 429 equations describing the ion trajectories in TWIMS, CCS cannot be directly measured from the 430 drift times, but they can be evaluated after calibration with ions of known CCS and under 431 defined conditions of the drift cell (gas pressure and temperature, drift cell length, wave height 432 and speed). Ruotolo et al. reported a detailed TWIMS calibration protocol to which readers 433 may refer to [28]. Generally, proper calibrants should cover the IM drift time range of the ions 434 of interest, but also being physically and chemically related to the analytes and best bracket 435 the $\mathrm{m} / \mathrm{z}$ of the compound to be analysed, especially when small differences in CCSs are 436 expected [29]. The most reliable calibration is obtained by using reference CCS values 437 obtained by DTIMS operating with nitrogen as buffer gas ( ${ }^{\left.\mathrm{DT} C C \mathrm{~S}_{\mathrm{N}}\right)}$, since TWIMS cannot 438 operate with helium and is more often commercialised with nitrogen, thus giving access to $439{ }^{{ }^{T W}}{ } C C S_{N 2}$ measures. The accuracy of CCS values is an important topic now that these values 440 are becoming an additional reference parameter in automated peak assignment of database 441 for-omics sciences.

442 Similarly, a calibration is needed for obtaining CCS values in DMA. As described in [30], a 443 single calibrant ion (such as tetraheptylammonium ${ }^{+}$) could be used to link the applied voltage 444 to the mobility of the ions transmitted, assuming that pressure and temperature inside the DMA 445 classification zone are known and controlled.

447 TIMS is another example of ion mobility analyser operating in the low field limit, but with no448 constant field. In TIMS, an axial electric field is applied to accumulate, trap and elute the ions 449 according to their $\Omega / z$ ratio (see section 1.5 for additional hardware details). Unlike the 450 configurations introduced previously, larger ions (high CCS) migrate first with respect to the 451 smaller ions (low CCS). Moreover, similar to TWIMS, CCS determination can be obtained only 
upon proper calibration with ions of known CCS as TIMS also operates with nitrogen as buffer

453 gas. Since the commercialisation of this analyser is still relatively recent, there are still very

454 few sources reporting ${ }^{\mathrm{TIM}} \mathrm{CCS}_{\mathrm{N} 2}$ values [5].

3.3. Prediction of collision cross section values

457 Even though the experimental CCS values represent an interesting structural parameter 458 obtainable by IMS, it is not sufficient to allow any structural interpretation on its own. To 459 disclose the conformation and shape adopted by the ions in the gas-phase, the experimentally derived CCS needs to be compared with computationally derived CCS values obtained from 461 atomistic models. Nowadays, this approach is the gold-standard if using IM-MS as analytical 462 technique for "conformation analysis" [2,4,5,31-41].

463 The first challenging task is the achievement of reliable atomistic models. A plethora of 464 computational approaches might be applied for this purpose, including quantum mechanical 465 (QM) methods such as (but not limited to) density functional theory (DFT), ab-initio or semiempirical methods and classical methods such as Monte Carlo (MC) and molecular dynamics 467 (MD). The method of choice is at the discretion of the user, but it is mainly driven by the size of the system under investigation. Generally, QM methods offer the highest level of accuracy, but they are extremely time-consuming and usually viable only for small systems, whereas 470 classical methods are instead more suitable for large systems such as biomolecules [42]. A

471 detailed evaluation of the computational approaches is beyond the scope of this review, but it 472 is worth noting that extensive proficiency in the field of computational chemistry is required to 473 obtain reliable atomistic models, thus several research groups (using IMS as structural tool) 474 have already taken into account the possibility to have a modeller in their group or to work in 475 close collaboration with a modelling team.

476 The second challenging task is the reliable determination of computationally derived CCS. 477 Indeed, once a pool of atomistic models, presumably representing the experimental gas-phase 478 conformations, have been selected through a proper computational method, a CCS calculation 479 algorithm needs to be applied to finally obtain theoretical CCS values (CCS THE $_{\text {or }}$ CCS CAL $_{\text {) }}$ 
which can be compared to the experimentally derived CCS (CCS EXP or CCS IMS). Several CCS

481 calculation models might be applied for this purpose. The "historical" models (still widely known 482 and used) are the trajectory method (TM) [43,44], the exact hard sphere scattering (EHSS) 483 [44], and the projection approximation (PA) $[43,45,46]$, all initially parametrised for helium as 484 buffer gas and available in the MOBCAL suite of programs [47]. In general, the three algorithms 485 allow simulation of the collisions between the buffer gas and the randomly oriented structure 486 of the ion under investigation. However, during the collisions, multiple scattering events and 487 long-range interactions also occur, and the three models differ in the assumptions and the 488 grade of approximations of all these events, as depicted in Figure 4. TM $[43,44]$ is an explicit 489 model which can be considered as the most rigorous and physically appealing method, since 490 it accounts for both long-range interactions and multiple scattering events. It is the method of 491 choice for small molecules calculations, but it becomes prohibitively slow if applied to large 492 molecules (> 1000 atoms), except if parallelization of computation is available. EHSS [48] is a 493 hard sphere collisions based method, meaning that both buffer gas and ion under investigation 494 are modelled as hard spheres. It computes the multiple scattering events, but it does not take 495 into account the long-range interactions, offering a reasonable balance between CCS accuracy 496 and time requested for the calculation, also for large molecules (even if an over-estimation of 497 CCS could arise in this latter case). Finally, PA $[43,45,46]$ is a non-explicit method that does 498 not consider the multiple scattering events, or long-range interactions, but works by projecting 499 the "shadow" of the ion onto a plane to find the average area of all the possible orientations of 500 the molecule under investigation. It is the fastest method, but can lead to a systematic 501 underestimation of the CCS, especially for molecules presenting cavities. PA is generally recommended for CCS determination of small molecules (10-200 atoms) with convex surfaces, even though a good correlation has been reported between TM and PA when dealing with

504 CCS calculation of macromolecules [49].

505 During the last few years, optimizations of these three algorithms and also their 506 parametrizations have been reported, especially to answer to the need of including nitrogen 507 as collision gas [50-54]. In the meantime, new algorithms have also been proposed, such as 
508 the projection superimposition approximation (PSA) [55-57], and the local collision probability 509 approximation (LCPA) [58], both non-explicit methods; the diffuse hard spheres scattering 510 (DHSS) [59] that is a hard sphere collisions based method; and the diffuse trajectory method 511 (DTM) [59] that is a physically explicit method. Furthermore, new CCS calculators are still 512 being developed with the goal to provide the most reliable CCS calculation possible, especially 513 for large biomolecules and with nitrogen as collision gas. This is the case of the IMPACT

514 software [49] that has been specifically developed for the CCS calculation of large 515 biomolecules. Indeed, IMPACT calculates CCS using an octree-augmented projection 516 approximation with an exceptional boost to speed for large targets. "Collidoscope" [60] is an 517 alternative TM based CCS calculator using parallel processing and optimized trajectory 518 sampling, implementing both helium and nitrogen as collision gas option.

519 It is hard to compare the performance of all these approaches, but accuracy and computing 520 time are clearly strongly dependent on the molecular size. What is clear is that this gold rush 521 indicates the increasing and intriguing involvement of IM-MS in the field of structural 522 characterization.

523

\section{Resolution and separation performance of ion mobility spectrometry}

4.1. Basic resolution equations in ion mobility

526 The resolving power of IM is defined in the same fundamental way as for mass spectrometry,

527 but using mobility related parameters (e.g. $t_{d} / \Delta t_{d}$ instead of $m / \Delta m$ ). IM resolution has also been 528 historically described using a variety of approaches beginning with chromatographically529 equivalent terms including plate height theory $[61,62]$. However, due to the fundamental 530 differences in ion mobility analyser principles, different models are necessary to describe the 531 achievable resolving power. As a number of recent reviews have comprehensively described 532 fundamentals behind IMS separation and resolution capabilities $[8,9,16]$, only key theoretical 533 equations for describing IMS resolution relevant to commercially available IM-MS 534 instrumentation and their underpinning studies are highlighted in this section. 
535 The nature of low-field drift tube ion mobility lends itself particularly well to understanding IM

536 resolution as the diffusion-based band broadening can be described by similar equations to 537 that used for isocratic linear chromatographic separations. Following historical development of 538 DTIMS instrumentation and seminal theory [27,63], the conditional resolution model [64] 539 provides a fundamentally-sound basis for describing the evolution of zone width in an ideal 540 case and the maximum obtainable sample peak capacity can thus be readily calculated 541 according to critical experimental parameters (e.g. drift gas, tube length, temperature, 542 pressure, sample type). However, limitations for this model are well-known, including the 543 influence of ion trapping and release processes, as well as additional broadening of the ion 544 packages between the IM and mass analysers. A semi-empirical model designed to account 545 for these effects was developed by the group of Hill [65] and offers a means to better 546 understand instrumental contributions to non-ideal performance, as shown in the recent study 547 of May et al. [66]. Resolution on current commercially available DTIMS instrumentation is in 548 the order of 40-60 for low pressure cells for singly charged compounds (Agilent) and up to 250 549 for high pressure cells (TOFWerk). In Table 2 [21,27,64-71], various aspects of these 550 experimental differences can be rationalised according to the relevant parameters influencing 551 DTIMS resolution. Nevertheless, attempts to improve resolution should also be considered 552 alongside other practical considerations. For example, use of above ambient pressure results 553 in improved resolution, but will also result in substantial losses in transmission [72].

554 In the case of TWIMS, the fundamentals and achievable resolution are described in detail in 555 the publication of Shvartsburg and Smith [67]. Unlike in DTIMS, the measured drift velocity is 556 proportional to the square of the ion mobility. This yields a resolution term that is dependent 557 upon the ion mobility (scaling as $K^{1 / 2}$ ) and allows the IM resolution to be practically optimized 558 according to the spread of mobilities to be measured. Further technical changes in the design 559 of commercially available TWIMS devices has allowed improved resolution (40-60) to be 560 achieved with consideration to CCS measurement stability and the optimization of the ratio of 561 wave height and wave velocity given thorough investigation $[17,68]$. Furthermore, exciting 562 developments in building serpentine paths of several meters in length seem to be the way 
563 forward to achieve resolution of several 100s for next generation TWIMS devices as effective

564 IM length can be increased enormously without increasing the instrument footprint [73].

565 A resolution equation for TIMS was derived by Hernandez et al., whereby the resolution for a 566 given ion is proportional to the gas velocity $\left(v_{g}\right)$ and to the fourth root of the length of the electric 567 field gradient [69]. As ions of different mobilities will be trapped using different gas velocities, 568 the achievable resolution will differ accordingly. Finally, the achievable velocity is primarily 569 controlled by $v_{g}$, the electric field ramp speed, and the RF confinement. Experimental studies 570 have demonstrated good agreement with this model and resolution of up to 300 under 571 optimized conditions [74]. However, it must be noted that this very high resolution is only 572 achievable for a narrow $\mathrm{m} / \mathrm{z}$ (respectively mobility) range. When used for generic, full mass 573 scan IM-MS experiments then the resolution will be in the range of 50-70.

574 Finally, as described in the review of May and McLean, IMS resolution has limitations as a 575 standalone metric for comparing different types of IMS analysers [9]. In particular, the 576 achievable resolution for DMA and FAIMS are more difficult to compare. An excellent overview 577 of DMA resolution theory is found in the aerosol science literature [75], while some recent 578 examples have demonstrated resolving powers in the order of 65 using DMA-MS with 579 optimized plate designs [76]. The fundamentals of compensation field-based resolving power 580 for FAIMS were outlined in a computational study from Shvartsburg and Smith [77] with some 581 recent contributions also highlighting some of the limitations of using classical resolving power 582 to describe the performance of FAIMS devices [78]. Although not implemented in routine 583 FAIMS analysis, one recent study demonstrated an improvement in the resolving power of 584 FAIMS from 20 up to $400-500$ by the use of optimized gas mixtures and extended separation 585 times [22], while another recent work utilized ion focusing strategies to yield up to $37 \%$ 586 improvement in resolution [79].

587 A summary of the most relevant IMS resolution equations is provided in Table 2.

588

$589 \quad$ 4.2. Separation performance in ion mobility 
590 Based on established differences in CCS values of molecules and IM resolution models, the 591 theoretical limitations of various ion mobility analysers can be considered according to the 592 instrument design parameters. Many early studies have assessed peak capacity in some form 593 for IM-MS analysis, with some of them focusing specifically on LC-IM-MS [80-82]. Akin to the 594 chromatographic definition [83], peak capacity within IM is simply a theoretical estimate of the 595 maximum number of peaks from the sample class of interest that can be fully resolved side596 by-side within a single scan. In combination with resolution and/or peak capacity models for 597 different types of ion mobility devices, improvements in separation potential can be modelled 598 and practically verified. An excellent example of the improvements from increasing path length 599 can be seen in the development of serpentine paths for TWIMS separation of ions [73]. With 600 effective lengths of several meters, quite elevated resolution separations have been realised, 601 while still maintaining sub-second IMS separation times.

602 One of the great promises of combining IM with MS is the possibility to resolve and individually 603 interrogate isobaric species present in a single sample. For such considerations, the IM 604 resolving power and measurement precision are the most critical factors. The majority of 605 publications on IM-MS continue to use experimentally determined IM resolving power as a 606 means to broadly describe instrument performance, but it is critical to point out that the nature 607 of the ion (charge state), ion gating/trapping, and the IM principle used can all influence the 608 measured resolving power for a given measurement. In many cases, more relevant information 609 for users can be gained by focusing on critical pair (peak-to-peak) resolution within the 610 molecular class of interest, as originally postulated by Karasek and Kim [84]. Practically, a 611 number of studies have demonstrated that isomers exhibiting minor differences in CCS can be 612 differentiated with excellent repeatability precision when analysed separately. Conversely, 613 measurements of mixtures of isomers often yield only broadened IMS peaks as the differences 614 in CCS are in the order of $<2 \%$ and cannot be resolved by most IM-MS instrumentation (Figure 615 5) [82]. In a recent contribution, the group of McLean provided theoretical estimations for the 616 required resolution to achieve sufficient IM separation such as small molecules and explored 617 the necessary advances in IM resolution required in some details [85]. In particular, the 
618 separation challenges associated with different isobaric separation problems are discussed

619 with reference to the degree of separation required. The differences in CCS values for isomer

620 pairs of particular classes (e.g. diasteroisomers) were estimated to vary from 0.3 up to $6.9 \%$, 621 and further suggest that an instrument with a resolving power of 250 should be capable of 622 resolving approximately three quarters of the considered isomer pairs. Thus, as modelling 623 developments and improvements in measurement accuracy established by inter-laboratory 624 comparisons continue to inform our understanding of IMS behaviour, we expect that instrument 625 design can mature in order to address this challenge. Nevertheless, it is clear that chemical 626 separation (e.g. LC) remains critical in many cases for the analysis of complex sample analysis 627 in order to correctly assign mass spectral features to individual compounds, and also to reduce 628 ion suppression which is obviously not mitigated by ion mobility separation.

\section{Formation of protomers - a potential issue in ion mobility spectrometry?}

631 When considering a singly charged species in positive electrospray ionization mode (ESI+), 632 the molecules have to be first protonated, and the added proton, which gives the charge, is 633 located at the molecule site having generally the largest gas-phase basicity. However, a significant number of substances possess several protonation sites, which can lead to different isomers in the gas phase, also known as protomers. As reported in [4], the average number of

636 protomers for more than 100,000 structures of biologically relevant compounds contained in 637 the drug data report database was equal to 6.3 per compound, which means that the 638 prevalence of protomers in the small molecule chemical space is important. In addition, it has 639 been reported that the protonation sites (location of charges) can have a significant effect on 640 the conformation of an ion [4]. In MS and HRMS, the protomers cannot be distinguished since 641 they possess different conformation, but the $m / z$ ratios remain identical. However, when adding 642 an IMS dimension to the separation, the protomers can be differentiated based on their shapes, 643 leading to several peaks observed on the mobilogram. This behaviour is well illustrated in 644 Figure 6, showing the mobilogram of ciprofloxacin (fluoroquinolone antibiotic), which reveals 645 the presence of two well separated gas-phase species (protomers), with their respective sites 
646 of protonation in the positive ionization mode (on the cyclic ketone and the piperazine moieties)

647 and their corresponding drift times [86]. In this example, these two different gas phase

648 components, although they only differ by their site of protonation, were well separated in IMS

649 (1.14 ms difference in drift time). The experimental IMS data of ciprofloxacin were compared 650 with theoretical calculations using molecular modelling (density functional theory) and

651 subsequent CCS calculations using projection approximation. Besides ciprofloxacin, there are

652 several other examples reported in the literature.

653 In differential mobility spectrometry, two protomers were observed for the 4-aminobenzoic acid

654 [87]. In this study, the authors have demonstrated that the relative abundance of each protomer 655 depends on the nature of the solvent employed in ESI. Various proportions of acetonitrile/water 656 and methanol/water were tested and the following conclusions were drawn: in presence of a 657 protic solvent $(\mathrm{MeOH})$, mostly O-protonation of 4-aminobenzoic acid occurs, while the 658 presence of aprotic solvent (acetonitrile) favours the formation of N-protonated 4659 aminobenzoic acid. This behaviour can be attributed to the stabilization of one of the two 660 protonated forms in a given solvent [88]. Similar observations on the influence of ESI solvent 661 on the relative abundance of protomers were reported for benzocaine, a local anaesthetic [89]. 662 The authors proved that the differences in drift times observed for the two species were 663 attributed to a different charge distribution within the molecules. They also highlighted that the 664 permittivity of the solvent was a key point since a highly protic solvent (such as $\mathrm{MeOH} /$ water) 665 facilitates the ionization of the most basic group (amide nitrogen) into the gas phase, while the 666 carbonyl oxygen becomes energetically favourable for protonation in the presence of a less 667 protic solvent (ACN/water), when the relative permittivity decreases.

668 Apart from differences observed between protomer abundance when altering the nature of the 669 ESI solvent, different fragmentation behaviours were also reported for the protomers. This has been highlighted in [87], where some differences in the amount of residual precursor ion and

671 in some fragment ions, as well as the presence of unique ions were observed for one of the 672 two protomers of 4-aminobenzoic acid. These differences in MS/MS spectra of protomers were 673 also highlighted in [90], with ciprofloxacin as a case study. The formation, separation and 
674 characterization of the protomers of aniline and three different porphyrins in presence of 675 different organic solvents and using polarizable $\mathrm{CO}_{2}$ as the drift gas was shown in [91]. The 676 observed protomers of aniline were characterized via structurally diagnostic collision-induced 677 dissociation (CID), thanks to post-TWIMS CID, while the pre-TWIMS CID allows the 678 determination of protomers ratio from equilibrium in solution or formed in the gas phase. For 679 aniline, the two protomers, namely N-protonated and unusual ring-protonated species, exist 680 as a ratio of 5:1 in solution equilibrium. This ratio was found to be substantially different in the 681 gas phase at non-equilibrium conditions. The same conclusions were drawn for porphyrins, 682 showing that pre- and post-TWIMS CID may be considered as a useful structural tool for 683 investigating protomers in more details.

684 In conclusion, IMS can be considered as a valuable analytical tool for the separation and 685 characterization of protomers. This feature could be particularly attractive for those who are 686 interested in determining the most favourable site of protonation or deprotonation of molecules, 687 which exhibit several acidic and basic sites. Therefore, the characterization of protomers is of 688 great fundamental relevance, to better understand compound stability and reactivity. However, 689 in analytical chemistry, the presence of various protomers for one single molecule is not 690 welcome, since it will surely add complexity to the mobilogram and MS spectra, making the 691 data interpretation difficult. Indeed, several peaks could potentially be observed for one single 692 molecule on the mobilogram. If these different species cannot be totally resolved in IMS, the 693 corresponding mobility peak will appear broad and distorted (shouldering) on the mobilogram. 694 Currently, the IMS resolution is relatively low (often below 50), and therefore, the different 695 protomers are often not separated at all and are analyzed as a single peak in IMS. However, 696 if (as expected) the IMS resolution will be significantly enhanced with the next generation of 697 instruments (see section 8.1.), numerous protomers will be observed for one given substance, 698 which will create some additional issues in terms of data interpretation. Finally, the number of 699 studies dealing with the understanding of protomers formation, separation and characterization 700 in IMS and its prevalence is still too limited and deserves some additional work from specialists. 
701 In addition, it is important to consider that the same effect (protomer formation) can also takes 702 place with proteins and protein complexes. This is of great interest, because of the mechanism 703 whereby charges migrate upon gas-phase unfolding of the protein (collision induced unfolding, $704 \mathrm{CIU})$. More details will be provided on this particular mechanism in section 7 , dedicated to the 705 characterization of large biomolecules.

\section{Application of ion mobility spectrometry to the analysis of small molecules}

6.1. Metabolomics and related applications

709 Global metabolomic studies are a challenging task that aims to analyze as much of the metabolome of interest as possible often within a single analysis [92]. For this reason, high resolution mass spectrometry in combination with chromatographic separation has become the essential tool for successful metabolite annotation and relative quantification tasks $[93,94]$.

713 IM-MS in combination with LC attempts to directly address the challenge of limited metabolome 714 coverage within a single analysis, by increasing overall method selectivity, which was outlined 715 in recent contributions on this topic $[9,95]$. The high chemical diversity of small metabolites 716 (e.g. encompassing amino acids, small organic acids, sugars, nucleosides, nucleotides, carbohydrates, sugar phosphates, vitamins, but also lipids) and their widely differing molecular properties (e.g. polarity, acid/base character) provides particular challenges for the

719 development of IM-MS and LC-IM-MS workflows as in-source fragments, dimers and adducts 720 can be separated by IM, which would not occur when using LC-MS alone. Thus, the correct 721 re-grouping of these signal features and interpretation of data from such workflows remains a 722 challenging task that has not yet been solved. The earliest literature examples using this combination for metabolomics demonstrated increased confidence in metabolite annotation via drift time or CCS alignment, but also limitations in transmission and assignment of 725 molecular formulae to adducts [72,96,97].

726 Building on a landmark interlaboratory study [98], Paglia and Astarita recently published a 727 protocol using LC-TWIMS-MS for both metabolomic and lipidomic studies using this platform, 728 which presents details on all procedures from calibration, tuning, analysis up to data 
729 processing including the derivation of ${ }^{{ }^{T} W} \mathrm{CCS}_{\mathrm{N} 2}$ values [3]. Other recent works focusing on 730 method development for global metabolomics have investigated the use of commercially 731 available TWIMS-MS instrumentation for urine profiling [99] and DTIMS-MS for human plasma 732 and HaCaT cells [100], whereby improvements in signal-to-noise and some confirmed 733 examples of isobar separations have been demonstrated. Other notable recent studies have 734 focused on non-targeted metabolomic assessment of placental samples [101], and serum 735 profiling [102]. The potential of LC-FAIMS-MS was also explored in a very recent contribution 736 with a broad outlook toward different "omics" approaches [103].

737 In addition to high resolution mass data, as commercially-available IM-MS instrumentation also 738 includes a quadrupole and collision cell (e.g. IMS-QTOF), the potential of using both IM-MS 739 with fragment ions associated to the precursor ion (i.e. aligned by drift time) can become part 740 of standardized workflows in order to utilize retention time, CCS, MS and MS/MS information 741 for metabolite annotation. However, data processing to make use of all of this information for 742 annotating metabolites in fully non-targeted workflows is time consuming and remains a 743 current roadblock for method development.

744 Aside from global metabolomic profiling, several groups have also focused on characterization 745 of secondary metabolites including food and plant studies [104-107]. Xiao et al. studied the 746 thermal degradation of beta-carotene using TWIMS-MS and putatively identified several 747 breakdown products [107]. Causon et al. demonstrated preliminary developments for wine 748 metabolomic studies using DTIMS, where excellent day-to-day repeatability precision $(<0.3 \%)$ 749 and agreement between CCS determined using standards and online measurement of real 750 samples $(<0.5 \%)$ was observed [104]. Covington et al. recently reviewed strategies utilized for 751 secondary metabolism and concluded that IM-MS is likely to play a role in natural products 752 dereplication [108]. Structural isomers and epimers of steroids, oxysterols and related 753 hormones have also been in focus within some recent IM-MS studies demonstrating the 754 suitability of available instrumentation for a diverse range of applications [109-111]. In all of 755 these examples, cleaner mass spectra and use of CCS as an identification point are 756 highlighted to have potential for increasing confidence in confirming metabolite identity. Thus, 
757 it is of critical importance for establishment of user-friendly LC-IM-MS metabolomic workflows

758 that the introduction of CCS as a conditional identification point for metabolite annotation

759 involves careful establishment of consensus CCS values to populate libraries for global use. It

760 is therefore expected that combined efforts in building reference CCS libraries for different

761 types of IM-MS instrumentation will emerge in order to provide validated methods supporting

762 metabolomic-related studies.

763 The area of drug metabolism has also been studied using IM-MS in recent contributions with 764 particular focus on isomeric variants. Reading et al. studied the gas phase conformation of 765 drug metabolites using TWIMS-MS, with a focus on correlating experimental data with 766 computational models for pharmaceutical structural characterization (Figure 7) [112]. Lee et 767 al. investigated metabolites of the anticancer drug Paclitaxel to study hydroxylation at different 768 sites using TWIMS-MS and supported their results with theoretical calculations [113]. Beucher 769 et al. studied non-steroidal selective androgen receptor modulators, which are prohibited by 770 the World Anti-Doping Agency, but remain readily available and potentially used in livestock 771 production for growth promotion [114]. Interestingly, the authors were able to successfully 772 couple supercritical fluid chromatography (SFC) separation with TWIMS-MS for the analysis 773 of bovine urine and suggested that CCS has potential within this workflow to be used as an 774 identification parameter within a screening method.

\subsection{Glycan analysis}

777 Glycans constitute a large class of biomolecules with a broad chemical diversity. They are

778 involved in many biological processes, such as embryonic development, cell-cell recognition, 779 inflammation and fertilization, among others [115,116]. Glycans can be covalently bound with 780 proteins to form glycoconjugates and hence affect protein folding mechanism and protein 781 stabilization. An abnormal conjugation between proteins and glycans can lead to a modification 782 of the tertiary structure having an impact on their biological response.

783 Structural characterization of glycans is the first step towards the understanding of their 784 biological activity. Due to their structural heterogeneity, the elucidation of glycan structures 
remains a challenging task. Liquid chromatography combined with tandem mass spectrometry

786 has been widely used for glycan analysis [117-119]. Nevertheless, different chromatographic

787 columns may be required to provide sufficient separation of isomers, which cannot be obtained

788 with tandem MS, leading to a low analysis throughput. Recent advances in IM-MS have

789 allowed the improvement of the structural characterization and separation of carbohydrates

790 have been reported since then $[5,120]$. Clemmer and coworkers first published the reduced

791 mobilities of negatively charged trisaccharides and their fragments by using a home-built drift

792 tube instrument [121]. In 2003, Hill and coworkers separated 21 different carbohydrate

793 standards using a non-commercial ion mobility instrument as well [122]. Overall, these results

794 showed the capabilities and the potential of IM-MS for the analysis of carbohydrates.

795 The interest on IM-MS especially increased after the advent of commercial instruments. A large

796 number of studies dealing with carbohydrate analysis in IM-MS have been reported [120] since

797 then [5]. Resolving power is the major issue of ion mobility in the analysis of carbohydrates.

798 Several strategies have been developed to circumvent this problem. Formation of non-covalent

799 complexes with metal ions has been largely used to induce alternative carbohydrate 800 conformations and thus, enhance isomer separation [123-125]. This approach has been used 801 for comparing the effect of different cations on the CCS of several milk sugar isomers [123], to 802 study the influence of the metal cation on carbohydrate structure. The sodium adducts 803 presented larger CCS compared to lithium adducts, but $\mathrm{Na}^{+}$was not found to be a suitable 804 cation to provide the best isomeric carbohydrate separation. Altogether, it was concluded that 805 separation of isomers does not only depend on cation radius, but also on the stereochemistry 806 of the compounds. Hoffman et al. studied a set of six trisaccharide isomers with different 807 composition in the non-reducing-end moiety [126]. IM-MS experiments were performed in 808 positive and negative ion modes to evaluate the influence of the ion polarity. In addition, two 809 kinds of trisaccharide adducts were formed with $\mathrm{Na}^{+}$and $\mathrm{Cl}^{-}$to improve the separation of the 810 precursor ions. Overall, IM provided a better separation of configurational and linkage isomers 811 in negative ion mode, compared to sodium adducts or protonated trisaccharides. Interestingly, 812 similar ATDs were observed for $[\mathrm{M}-\mathrm{H}]^{+}$and $[\mathrm{M}-\mathrm{Na}]^{+}$sodium adducts, whilst an impact on the 
813 general structure of trisaccharides was observed upon chloride adduct formation. Another

814 additional interest about IM highlighted in this work was its ability to quantify small amounts of

815 carbohydrate impurities from a given mixture. Product ions in a relative concentration of $0.1 \%$

816 could indeed be efficiently detected and quantified by analyzing the corresponding ATDs.

817 The versatility of certain IM-MS instruments allows the fragmentation of ions before the IM cell

818 (pre-mobility CID) $[127,128]$ and/or after (post-mobility CID) $[129,130]$ to provide in-depth

819 insights about the structure of precursor carbohydrates and also to generate specific tandem

820 mass spectra. For instance, oligosaccharide-alditols from bovine sumaxillary mucin (BSM)

821 were separated by HPLC and analyzed by IM-MS in the negative ion mode [128]. Some

822 specific precursor ions were post-mobility fragmented to generate specific tandem mass

823 spectra from isomeric precursor ions. CID was also performed upon IM separation giving rise

824 to isomeric mobility peaks. The mobility resolved product ions were also fragmented in the

825 transfer cell, generating further structural information. A more recent study from Harvey et al.

826 performed a post-mobility fragmentation of hybrid and complex glycans obtained from five

827 different glycoproteins [130].

828 The previous works were mainly focused on improving the separation and identification of 829 isomeric species and the characterization of the global structure from IM measurements. 830 However, IM can also be used as a filter, to reduce the complexity associated with the 831 carbohydrate mass spectra $[11,127,130,131]$. Interactions between neutral buffer gases and 832 ions strongly depend on the chemical nature of the analytes, leading to different mobility 833 behaviors. May et al. calculated almost 600 CCS values that comprise different molecules 834 such as lipids, peptides, carbohydrates and quaternary ammonium salts, using a drift tube 835 coupled to a quadrupole time-of-flight mass spectrometer [11]. Above $1200 \mathrm{Da}$, these four 836 classes exhibit different gas-phase packing trends that were clearly distinguishable by IM. In 837 addition, multiply charged ions will exhibit higher mobilities than singly charged ones, 838 describing specific drift time/mass-to-charge trends that can be individually extracted from the 839 global mass spectrum. This example is clearly observed in the analysis of $\mathrm{N}$-glycosides from 840 bovine fetuin studied by Harvey et al. in the negative ion mode [130]. Several well-defined 
841 regions are displayed in the driftscope, corresponding to different $\mathrm{N}$-glycan charge states. It is

842 worth pointing out that filter-role of ion mobility not only facilitates the interpretation of mass

843 spectra but also allows the detection of minor species that could not be observed in classical

844 MS conditions by improving the signal-to-noise ratio.

845 Alternative fragmentation techniques to CID are required to produce more diagnostic cross-

846 ring fragments such as electron excitation dissociation (EED) [132], vacuum ultraviolet

847 photodissociation (VUVPD) [133], and extreme ultraviolet light (XUV) [134] or spectroscopic

848 techniques such as infrared multiple photon dissociation (IRMPD) [78], to produce

849 characteristic IR spectra to elucidate the structure of the carbohydrate isomers. Further 850 improvements in separation and identification of glycan isomers can be achieved by coupling 851 two IMS dimensions, as recently demonstrated [135,136]. IMS-CID-IMS-MS experiments can

852 be performed with these experimental set-ups to study the structural heterogeneity of complex 853 glycan mixtures, where the separation of the precursor ions is not fully achieved in classical 854 IM-MS experiments.

\subsection{Lipid analysis}

857 Lipids occupy a distinct conformational space in IM-MS analysis, due to their relatively high 858 flexibility and low density (i.e. large CCS for their $\mathrm{m} / \mathrm{z}$ ). Moreover, aside from the broad class 859 separation requirements for lipid analysis (triacylglycerides, phospholipids, free fatty acids, 860 ceramides, sphingomyelins,...), there is great structural diversity within individual classes 861 influencing their conformation including different alkyl chain lengths, degree of saturation, and

862 double bond position. In this regard, IM-MS offers great potential to investigate this diversity 863 that may not be revealed by conventional analysis. Application of IM-MS to lipidomics has been 864 recently reviewed by Paglia et al $[137,138]$ and the reader is referred to these two contributions 865 for a comprehensive overview of this topic. Astarita and Paglia also recently published an 866 advanced lipidomic protocol using TWIMS-MS in combination with liquid chromatography [3], 867 while excellent insight into CCS calibration strategies for lipidomics using this type of 868 instrument was recently published [139]. In a particularly novel approach, the group of Murphy 
869 has demonstrated the use of product ion mobility for lipidomic workflows, whereby collisional 870 activation is used to generate product ions for which a CCS value can be determined to aid 871 with lipid identification using a TWIMS-MS platform [140,141].

872 Some very recent results from DTIMS studies have indicated the challenges associated with 873 separating minor differences in CCS (e.g. double bond position isomers) due to the sub-1\% 874 differences in CCS of many isomers. Kyle et al. studied a wide range of lipid classes using the 875 Agilent DTIMS instrument demonstrating the possibilities for a wide range of lipid isomer types 876 and the separation potential offered with current resolution limitations [142]. Dried blood spots 877 were the focus of a second study from this group involving longitudinal samples from diabetic 878 patients also assessed by DTIMS [143]. Stable isotope-labelling was used for profiling of fatty 879 acids using DTIMS, where the similarity of drift times was used to help associate labeled with 880 unlabeled acids in normal and cancerous human thyroid tissue samples [144]. Impressive 881 resolution of acyl chain- and double bond positional isomers of lipids has been demonstrated 882 on the TOFWerk DTIMS platform [145]. A resolution of $>250$ was calculated for isomeric lipids 883 measured under low-field conditions with the instrument operated with Hadamard multiplexing, 884 which requires sophisticated signal deconvolution approaches.

885 As is the case for metabolomic studies, standardization of CCS values must become a key 886 focus for building of global lipid libraries for identity confirmation and is already a targeted focus 887 of some computational modelling studies [146]. Nevertheless, some of the subtleties of gas888 phase lipid conformation have not yet been resolved between theoretical and experimental 889 considerations and ongoing effort is needed to reconcile these aspects. The commercial 890 availability of high-precision instrumentation utilizing different types of IMS analysers is already 891 playing a key role in supporting such work.

892 FAIMS offers a complementary approach in comparison to TWIMS and DTIMS and has also 893 been used for targeted studies of lipids by several authors for interrogating isobaric lipid 894 species as essentially a "mobility filter" prior to MS experiments. In one such study, Steiner et 895 al. used differential-mobility in combination with a state-of-the-art ozonolysis strategy to probe 896 the structure of deoxysphingosine [147]. This type of study underlines the structural diversity 
897 within a single lipid class that can influence biological behavior that can benefit from IM-MS as

898 a powerful analytical approach (Figure 8). A very recent study has also demonstrated some 899 promising results using FAIMS for resolution of a wider range of isomeric lipid classes [148]. 900 Finally, due the complementarity of different available IM-MS analysers, the potential for 901 revealing previously unknown biological information using IM-MS has started to emerge in 902 recent literature and further insightful publications in this area are expected.

903

904

\subsection{Environmental analysis}

IMS has a long history for usage in field applications entailing rapid environmental assessments. The recent review of Marquez-Sillero et al. presents IMS applications focusing on environmental analysis [149]. While IMS alone has great potential for applications requiring portable analysis, IM-MS and LC-IM-MS have excellent potential to be used for lab-based

909 screening workflows, particularly for trace analysis in complex samples. The group of Schmitz 910 has recently demonstrated a powerful approach by combining two online separation methods 911 (LC+LC or GC+GC) with DTIMS-MS [150,151]. Complex wastewater samples were 912 successfully screened using an in-house CCS database with excellent repeatability of CCS 913 and mass spectra, while their more recent study used GC+GC with atmospheric pressure 914 chemical ionization (APCI) for the screening of various compounds, including pesticides.

915 Further discussion of the potential of multidimensional chromatography in combination with

916 IM-MS is presented in section 8.3. Grimm et al. used TWIMS-MS for the study of of unknown 917 or variable composition biological materials (UVCBs), complex reaction products, and 918 biological materials in petroleum samples [152]. This workflow allowed mass-to-charge, drift 919 time, and Hydrogen/Carbon ratio to be used to support compound classification and inter920 samples comparisons. While such studies offer exciting results, for such approaches to 921 become broadly accepted in standardized methods, CCS will require standardization as an 922 identification point in order to be used alongside retention time and MS identification 923 parameters within regulated methods. 
924 The substantial challenge of exposomic studies, where 1000s of compounds including 925 xenobiotics, secondary metabolites, and transformation products which remain currently 926 unidentified, is a focus of high-throughput IM-MS studies whereby computational prediction of 927 CCS values is suggested to be a way forward to allow rapid screening of samples [153]. Such 928 a workflow requires substantial computational effort to predict CCS values, but some authors 929 have presented promising results using different approaches in addressing this major 930 challenge [154-156]. The workflow demonstrated by Zhou et al [156] was particularly in-depth 931 as DTIMS experimental data from a total of $\sim 400$ compounds were used to generate molecular 932 descriptors to train a machine-learning algorithm, which matched CCS values measured on 933 their instrument with a median relative error of $3 \%$. Alternative approaches to modelling of CCS 934 are discussed in Section 3.3.

935

936 6.5. Clinical analysis

937 A number of studies have investigated the use of IMS and IM-MS for the analysis of samples 938 of clinical origin and the potential of these technologies for such applications was recently 939 reviewed by Chouinard et al. [110]. As is the case for environmental applications, standalone 940 IMS instrumentation is considered to be attractive for clinical applications due to its portability, 941 ease of use and low cost. Rapid sampling of exhaled breath is one example that has already 942 explored the use of this technology since the 1980s [157]. In the case of IM-MS, some of the 943 more promising investigations for clinical applications appearing within the last few years are

944 aiming toward the use of IM-MS for imaging using desorption electrospray ionisation (DESI) or 945 matrix-assisted laser desorption ionisation (MALDI) in combination with IM-MS [158-160]. 946 Apart from these developments, many of the goals and challenges within clinical applications 947 using IM-MS also overlap with those of the related "omic" studies, which are presented in the 948 elsewhere relevant sections of this review.

950 7. Application of ion mobility spectrometry to the characterization of large $951 \quad$ biomolecules 
7.1. Separation of isobaric peptides

953 One of the most important challenge of structural biology is the elucidation of the protein 954 structure/function relationship. Posttranslational modifications (PTM) like phosphorylation, 955 acetylation, or methylation can be involved in modification of the protein structure and thereby 956 have a straight impact on their function. Identification and precise location are essential 957 information to provide a thorough PTM characterization. For this purpose, LC has been used 958 prior to MS/MS to separate precursor peptides, however this approach often fails to separate 959 and identify positional variants, especially when isobaric peptides exhibit no specific 960 reporter/diagnostic fragments [154]. A newer approach based on the implementation of IMS 961 has been used to provide an additional separation dimension to the characterization of large 962 biomolecules. Ruotolo et al. studied [154] a large set of phosphorylated and non963 phosphorylated peptides using a MALDI-DT-MS mass spectrometer. The contour plots show 964 that phosphorylated peptides exhibit lower drift times compared to non-phosphorylated 965 peptides with the same $\mathrm{m} / \mathrm{z}$. Unfortunately, the resolving power of the technique [161-163] 966 only allows the efficient separation of some phosphorylated variants. The separation capability 967 of IM was further improved by using FAIMS coupled to MS [23,164]. Three different 968 phosphorylated peptides from $\tau$ protein (VAVVRTpPPKSPSSAK, VAVVRTPPKSpPSSAK, VAVVRTPPKSPSpSAK) were efficiently separated under the influence of the asymmetric electric field and by modifying the $\mathrm{He} / \mathrm{N}_{2}$ ratio of the buffer gas [165]. The best separation was

971 achieved with a mixture of gas containing $40 / 60 \mathrm{v} / \mathrm{v}$ of $\mathrm{He} / \mathrm{N}_{2}$. The identification of each 972 phosphorylated variant was performed after ETD fragmentation. It is worth noting that ETD 973 spectraof each individual phosphorylated peptide do not exhibit unique fragments that allow 974 unambiguously identification of the precursor ions, thereby, an efficient separation is required 975 prior to ETD fragmentation. More challenging PTM characterization was envisaged with the 976 same experimental set-up, like methylated [166] and acetylated peptides [167]. Both 977 modifications were studied on histone tails, more precisely in the 1-21 and 21-44 segments of $978 \mathrm{H} 3$ tails, and 1-25 segment of $\mathrm{H} 4$ respectively. Positional variants of methylated and acetylated 979 peptides were separated using different $\mathrm{He} / \mathrm{N}_{2}$ or $\mathrm{H}_{2} / \mathrm{N}_{2}$ gas ratios, to improve ion separation. 
980 Overall, very narrow peaks and a baseline separation could be achieved after addition of He

981 to the buffer gas (from $0 \%$ to $40 \% \mathrm{v} / \mathrm{v}$ ). It is worth mentioning that methylated or acetylated 982 peptides are +14 and +42 Da modification, respectively over "middle down" sequences (up to $9833 \mathrm{KDa}$ ) which means that methyl variation accounts for $0.5 \%$ of the mass, requiring high 984 resolving power to provide an efficient separation. These results show the utility of IM-MS to 985 improve detection of positional isomers of modified peptides and identification from a complex 986 peptide mixture.

7.2. Adding an ion mobility dimension for improved peak capacity in high throughput large scale proteomics

Modern proteomic analysis consists of identification and simultaneous quantification of 991 thousands of proteins present in complex protein mixtures, with many applications in molecular 992 and cellular biology [168-170]. Up to now, most proteomic studies rely on bottom-up analysis: 993 complex protein mixtures are digested into peptides that are further analyzed by reversed 994 phase liquid chromatography coupled to nanoelectrospray ionization tandem mass spectrometry (nanoLC-MS/MS) platforms. Hybrid tandem mass spectrometers such as ion trap-orbitrap, quadrupole-orbitrap and quadrupole-time-of-flight (QqTOF) instruments have dominated the field of large scale high throughput proteomics. These configurations offer high data acquisition speed and high mass accuracy for peptide precursor and fragment ions.

999 However, this classical bottom-up proteomic workflow presents a series of caveats : i) several 1000 peptides can co-elute from the chromatography column and ii) MS/MS acquisitions are mostly 1001 performed in a data-dependent (DDA) way, meaning that most intense ions are fragmented one at a time, the others being entirely discarded. Limited scan speeds and sensitivities of the mass spectrometers mostly account for this under-sampling, leading to subsequent lower

1004 sensitivities. Thus, improving the performance of new instrumentation relies on increasing the 1005 rate of MS/MS acquisition without information loss (transmission) on tandem mass spectra. 1006 Although ion trap instruments may still offer higher absolute sensitivity owing to their ability to 1007 accumulate precursor ions to a desired level prior to fragmentation (a feature that is not 
1008 available on QqTOF instruments), TOF instruments have the potential for very fast data 1009 acquisition (up to $100 \mathrm{~Hz}$ for QqTOFs) [171] and high in-spectral dynamic range, both of which 1010 are highly desirable for the analysis of the very complex samples [172]. IMS can then be 1011 exploited to increase speed, selectivity, and sensitivity of MS/MS experiments $[2,173,174]$.

1012 The integration of TWIMS into DDA acquisition schemes [14], termed high definition or HD1013 DDA [175] has removed the long standing limitation of low duty cycles of QqTOF instruments 1014 and leads to considerably improved sensitivity and productivity of proteomic analysis on such 1015 instruments [14]. The actual speed of HD-DDA is $30 \mathrm{~Hz}$, mainly owing to the time required for 1016 on-the-fly data processing within the instrument control software. In the TWIMS configuration, 1017 charge separation capabilities of TWIMS cells are used to separate singly charged from 1018 multiply charged ions of tryptic peptides.

1019 Data-independent acquisition (DIA) modes, in which fragment ion spectra of all detectable 1020 precursor ions within an LC-MS run regardless of any specific characteristics (intensity, charge 1021 state and so on) are generated, [176] have also been reported as alternatives to circumvent 1022 under-sampling of MS/MS experiments. $\mathrm{MS}^{\mathrm{E}}$ is one of the first reported DIA approach and 1023 consists of two alternating MS scans recorded at low and high collision energy across the full 1024 mass range, providing precursor and product ion information, respectively [177]. The 1025 integration of IMS to DIA workflows has been referred to as high-definition $\mathrm{MS}^{\mathrm{E}}\left(\mathrm{HDMS}^{\mathrm{E}}\right)$. IMS 1026 provides here an additional dimension of separation that increases the overall system peak 1027 capacity in LC-MS workflows [174], offering the possibility to fragment precursor ions after IMS. 1028 IMS not only allows the resolution of co-eluting isobaric species, but also enables the use of 1029 drift-time information [178] for the alignment of precursor and product ions (in addition to 1030 retention times in chromatography). This drastically increases the number of identified peptides 1031 and proteins in complex samples as compared to $\mathrm{MS}^{\mathrm{E}}$ workflows without IMS [179]. Optimized $1032 \mathrm{HDMS}^{\mathrm{E}}$ approaches achieved similar proteome coverage as compared with state-of-the-art 1033 DDA methods and displayed excellent reproducibility and overlap between technical replicates $1034[180,181]$. Figure 9 presents an example of $\mathrm{HDMS}^{\mathrm{E}}$ analysis of a $400 \mathrm{ng}$ Hela digest, leading 1035 to the identification of more than 38440 peptides corresponding to 3490 unique proteins. 
1036 Thorough overview of the use of IMS-DIA-based label-free quantification and its applications

1037 in biomedical research and clinical proteomics have been reported elsewhere $[182,183]$.

1038 Similarly, IMS-DIA has also been reported for enhanced impurity detection in protein 1039 biotherapeutics (Host Cell Proteins, HCP), as they present a high dynamic range between the 1040 very abundant therapeutic protein and its low abundance impurities. HCPs present at a 1041 concentration as low as $1 \mathrm{ppm}(1 \mathrm{ng} \mathrm{HCP} / \mathrm{mg} \mathrm{mAb}$ ) were successfully identified and quantified 1042 in the reference NIST monoclonal antibody and Infliximab [13]. Recently, Haynes et al. 1043 described the use of a new mode of IMS-DIA acquisition: instead of performing IMS-DIA at a 1044 fixed velocity TWIMS settings, ramping the traveling-wave velocity increased drift space occupancy, amplifying resolution by $16 \%$, peak capacity by nearly $50 \%$, and peptide/protein identifications by $40 \%$, opening new perspectives for variable-velocity TWIMS in proteomics 1047 analysis [184]. To summarize, IMS adds an orthogonal analytical dimension, reduces ion 1048 interference to improve reproducibility, peak capacity, and peptide identifications to rival 1049 modern hybrid quadrupole orbitrap systems.

1050 A variant of the HDMSE approach has been proposed using a TIMS device [185], introducing 1051 the concept of parallel accumulation-serial fragmentation (PASEF) consisting of sub1052 millisecond quadrupole serial selection and fragmentation of multiple precursors in single 50 1053 ms TIMS scans [185]. PASEF enables hundreds of MS/MS events per second at full sensitivity. 1054 The PASEF workflow presents a series of advantages among which: i) no loss of sensitivity, 1055 ii) the resulting spectra are fully precursor mass resolved and iii) good compatibility with 1056 reporter ion based chemical multiplexing strategies (such as iTRAQ or TMT). A 10-fold gain 1057 should be achievable by PASEF in shotgun proteomics experiments, as a consequence of 1058 increased sequencing speed, without a decrease in sensitivity. However, modeling suggests 1059 that a combination of targeting more precursors and targeting weak precursors repeatedly will 1060 be most effective. The PASEF principle could be applied to any IM-MS configuration with the 1061 required sub-millisecond scan speed in the MS read out.

$1063 \quad$ 7.3. Ion mobility spetrometry for the characterization of gas phase protein structures 
1064 ESI-IM-MS has been mostly used to study the 3D architecture and conformational properties 1065 of many proteins and biomolecular complexes, through the measurement of their averaged 1066 CCS [28]. However, over years, the question "is protein conformation altered from the solution 1067 to the gas phase of the mass spectrometer?" became central to avoid misinterpretation of IM 1068 data. Several studies that compared IM measured CCS to either calculated CCS from PDB 1069 structures or in vacuum molecular dynamics (MD) generated CCSs brought partial answers to 1070 this question. One common observation is that when the charge increases, the molecules 1071 undergo Coulomb-induced unfolding to adopt extended helical structures [186,187] and 1072 ultimately string-like extended structures at very high charge states [188,189]. At low charge 1073 states, however, the proteins usually remain compact, with sizes that are expected for 1074 condensed phase native structures. Thus, depending on the type of proteins/complexes of 1075 interest, the gas phase collapse and/or unfolding of proteins is more or less pronounced. It is 1076 now generally accepted that 3D structures of globular proteins (like TRAP, TTR, SAP, integral 1077 membrane proteins) [190] survive the transfer into the gas phase without major compactions 1078 or unfolding at low energies [191], as attested by the number of success stories based on 1079 agreement between IMS-measured CCSs and calculated ones. More recently, Devine et al. 1080 [192] showed that non-globular proteins (like monoclonal antibodies or proteins with flexible 1081 hinge regions in between more structured domains) and RNA molecules undergo strong 1082 compaction in the gas phase (CCS values measured underestimate the physical size of the 1083 solution structure and modeled data of the biomolecule), highlighting a potential caveat in 1084 studying these specific biomolecules with this technique. However, IMS is not directly sensitive 1085 to protein secondary structure, and observing a compact structure does not guarantee that the secondary structure accurately reflects that of the native form. To go ahead with keeping 1087 secondary structures from the solution to the gas phase, optical/IR spectroscopy, a direct method that allows distinguishing helices from $\beta$-sheets, has been coupled to IMS [193]. In this 1089 configuration, IMS is used to select the global shape of proteins, followed by IR spectroscopy 1090 to probe their secondary structure. On two reference proteins, myoglobin (85\% of $\alpha$-helix) and $1091 \beta$-lactoglobulin (60\% $\beta$-sheets), IMS combined to IR showed that secondary along with tertiary 
1092 structures are retained in the condensed gas-phase. When IMS is combined to gas-phase 1093 infrared multiple photon dissociation (IRMPD) spectroscopy, gas-phase Coulomb-driven 1094 unzipping to a defined secondary structure is a general feature of all highly charged protein 1095 ions [194].

1096

1097

7.4. ion mobility spectrometry for proteins and protein-ligand complexes

1098

1099

7.4.1. Ion mobility spectrometry for intrinsically disordered proteins

Intrinsically disordered proteins (IDPs) have been the scope of many IM-MS studies (for review see $[195,196])$. Indeed IDPs, which are associated with a wide range of major disorders including cancer and amyloid-related disorders, are flexible proteins that can adopt many different conformations in order to bind to different physiological partners. This conformational 1103 change along with absolute conformation(s) characterization and detection of populations of 1104 conformations can be uniquely monitored by IM-MS. Bernstein et al. [197] performed IM-MS 1105 experiments on $\alpha$-synuclein that indicate how the size of its conformations differed with charge 1106 state at initial solutions of neutral and acidic $\mathrm{pH}$. IM-MS has also been used to study the 1107 structure of the DBD (DNA-binding domain) of the tumour-suppressor protein p53 with and 1108 without the functional zinc [198]. IM-MS showed smaller CCSs (12.3\% in CCS) for the p53 1109 without zinc than for the zinc-bound form. Other research using IM-MS to study IDPs has 1110 examined changes in conformational equilibria of the intrinsically disordered HMGA (high1111 mobility group A) chromatin factors [199]. IM-MS measurements of wild type and of a C1112 terminal truncated (shorter) HGMA2 were recorded. Despite the increase in size of WT with 1113 respect to the C-terminal truncated HGMA2, IM-MS revealed a more compact structure for the 1114 C-terminal shorter form, indicating that the presence of the C-terminal tail is here responsible 1115 for further compacting HGMA2, which could be correlated to a reduction in oncogenic activity. 1116 D'urzi et al. reported the use of IM-MS to characterize the complex between the intrinsically 1117 disordered $\mathrm{N}_{\text {TAlL }}$ domain and the phosphoprotein $\mathrm{X}$ domain $\left(\mathrm{P}_{\mathrm{XD}}\right)$ from measles virus (MeV). 1118 IM-MS revealed distinct conformers, yielding the estimation of the solvent-accessible surface 1119 area (SASA) in solution and the average CCS in the gas phase. Computational modeling of 
1120 the complex in solution, based on experimental IM-MS constraints, were generated and

1121 provided the first attempt to modeling the entire $\mathrm{N}_{\text {TAIL }}$ domain bound to $\mathrm{P}_{\mathrm{XD}}$ at atomic resolution.

1122 IM-MS is also well-positioned to assess IDP/ligand interactions and conformations induced 1123 upon ligand binding. Dickinson et al. have demonstrated the use of IM-MS to characterize the 1124 effect of two inhibitors RITA and Nutlin-3 on their respective binding partners: p53 and MDM2 1125 [200]. While no Np53:RITA complex was observed in native MS experiments, an alteration in 1126 the conformational distributions (through variations in ${ }^{\mathrm{DT}} \mathrm{CCS}_{\mathrm{He}}$ values) adopted by $\mathrm{Np53}$ in the 1127 gas phase was observed. This finding supports the hypothesis that RITAs mode of action 1128 proceeds via a conformational change in $\mathrm{p53}$, which was further confirmed by a combination 1129 of complementary biophysical techniques. Similarly, DTIMS analysis revealed that, in the 1130 absence of Nutlin-3, every charge state of N-MDM2 shows at least two conformations. Again, 1131 upon binding to Nutlin-3, the protein undergoes a compaction event similar to that observed 1132 with RITA and Np53. This multi-technique approach highlights the inherent disorder in these 1133 systems; and in particular exemplifies the power of IM-MS as a technique to study transient 1134 interactions between small molecule inhibitors and IDPs. For instance, Knapman et al. 1135 reported the use of IM-MS to probe the conformational characteristics of two IDPs, apo1136 cytochrome $c$ and apo-osteocalcin, which are both structured when bound to their respective 1137 substrates, but disordered in their unbound-states [201]. In both cases, the intrinsically 1138 unstructured apo-states exist as multiple conformations with larger cross-sectional areas than 1139 their holo-analogues, suggesting that IDPs do not preclude the formation of preferred 1140 conformations. Together, these examples illustrate the scope and utility of IM-MS for studying 1141 the characteristics and properties of IDPs, whose analysis by other techniques is limited.

1142 Another feature that can be monitored by IM-MS is the tendency of IDP to form amyloid fibrils, 1143 the protein aggregates involved in the onset of neurodegenerative diseases such as 1144 Parkinson's and Alzheimer's (for review see [202]). Due to their dynamic, polydisperse, and 1145 polymorphic nature, these oligomers are very challenging to characterize using traditional 1146 condensed-phase methods. IM-MS based approaches can be used to study amyloid 1147 formation, providing information about the assembly pathway and the size and shape of 
1148 presumably toxic oligomers. IM-MS was first used to separate, analyze and measure CCSs of 1149 individual oligomer species present in equilibrium [202]. A remarkable IM-MS study is reported 1150 by Leney et al. [203] and compares the oligomers formed from wild-type $\beta 2 \mathrm{~m}$ (beta-21151 microglobulin) with those formed from a variant of the protein ( $\mathrm{H} 51 \mathrm{~A})$, during in vitro fibril 1152 assembly. While H51A was shown to exhibit a two-fold increase in the lag-time of fibril 1153 formation, no differences were observed by IM-MS for the oligomers of the two proteins (similar 1154 CCSs). Real-time native MS was the only technique able to highlight the differences in the 1155 kinetics of the lag phase between the two protein variants. Similarly, IM-MS was used to 1156 investigate the formation of heterogeneous pre-fibrillar, oligomeric species involving human 1157 amylin (also known as islet amyloid polypeptide, hIAPP) and the amyloid-beta peptide (Aß1158 40), two pathogenic factors in Type 2 diabetes mellitus (T2DM) and Alzheimer's disease (AD), 1159 respectively [204]. Oligomerization of human islet amyloid polypeptide (IAPP), being 1160 considered as a pathogenic process in type II diabetes, has also been extensively investigated 1161 by IM-MS $[205,206]$. Time-course MS and IM-MS were reported to probe the early aggregation 1162 states of an amyloidogenic endecapeptide derived from amino acid residues 105-115 of the 1163 human plasma protein transthyretin [207]. Early $A \beta$ oligomers, which are thought to be 1164 responsible for the neurodegeneration of AD patients, have also been analyzed using IM-MS 1165 [31]. IM-MS revealed for instance that full-length $A \beta 42$ aggregates faster and is more cytoxic 1166 than the shorter $A \beta 40$ variant, which could be related to a compact stable tetramer formation 1167 for the $A \beta 40$ and a rather "open tetramer" that can evolve to an hexamer for the $A \beta 42$. More 1168 recently, Illes-Toth et al. have characterized by IM-MS a specific population of $\alpha$-syn oligomers, 1169 which was shown to have the ability to induce intracellular aggregation [208]. Structurally, the 1170 MS compatible oligomers populated a range of species from dimers to hexamers. Lower-order 1171 oligomers were structurally diverse and consistent with unstructured assemblies. Higher-order 1172 oligomers were shown to be compact with ring-like structures. The observation of this compact 1173 state may explain how this natively disordered protein is able to transfer pathology from cell to 1174 cell and avoid degradation by cellular proteases. 
1175 IM-MS provides valuable data on the ion's overall size and shape, but it does not yield direct

1176 information about the underlying internal structure. To obtain such data, IM-MS was recently 1177 used as a preselection tool for a further analysis using orthogonal, structure-sensitive gas1178 phase techniques, like action fluorescence resonance energy transfer (FRET) spectroscopy 1179 [209] or gas phase infrared spectroscopy [210]. IM-MS can also be successfully applied for 1180 high-throughput inhibitor screening. IM-MS is capable of high-throughput binding screening 1181 with a rate of up to 5000 molecules per day [211,212], providing not only conformational 1182 characteristics but also binding stoichiometries and specificities.

1183 To summarize, IM-MS provides a useful insight into events that occur during the early stages 1184 of aggregation including delineating the structure of the monomer, identifying oligomer 1185 distributions, and revealing mechanistic details of the aggregation process.

7.4.2. Ion mobility spectrometry for protein-ligand interactions

1188 The study of protein-ligand interactions is of particular interest for the pharmaceutical industry, 1189 especially in the context of drug development. Native MS has there also been demonstrated 1190 to be a pivotal technique providing relevant information about stoichiometry of interaction, site1191 specificity of ligand-binding and, in appropriate cases, affinities (through dissociation constant, $1192 K_{D}$, measurements) between the target protein and the ligand [212,213]. As the binding of 1193 ligands often induces a conformational change of the protein, the hyphenation of IMS to native 1194 MS could bring an additional level of conformational characterization, in addition to the 1195 classical binding screening (for review see [213,214]). A first solution to track the 1196 conformational change of a protein upon ligand binding is thus to perform IM-MS and measure 1197 CCS values for the free protein and the ligand-bound form. However, this simple and basic 1198 idea is not straightforward as ligand binding not always induce major global conformational 1199 changes, but rather very localized conformational changes with exceedingly low impact on the global CCS measurements (below 5\% CCS differences). In addition, the resolution of the IMS 1201 cell is here of paramount importance. As a consequence, careful optimization of the IM cell to 1202 distinguish between protein $(P)$ and protein:ligand $(P: L)$ are required to resolve CCS changes 
1203 lower than $2 \%$ for P:P and P:L complexes [29,68,215]. Despite these limitations, the use of IM1204 MS to discern conformational tightening on the c-MYC:MAX leucine zipper formation due to 1205 ligand binding has been previously reported by Harvey et al. [216] and along with these 1206 findings, provides an exciting prospect as a method for screening inhibitors to conformational 1207 dynamic systems. Collision induced unfolding (CIU) experiments have proven to be an 1208 interesting IM-based alternative to tackle systems involving very subtle conformational 1209 changes $(<2 \%)$. CIU is an IMS-based gas phase method that aims at collisional heating of 1210 ions, typically through the application of an accelerating electric field that results in higher1211 energy collisions with background gas. Ions undergo unfolding and the resulting conformers 1212 are separated in the IMS cell. Two ions that share similar initial CCS values can be readily 1213 differentiated through the CCSs and collision energies associated with the unfolded protein 1214 forms populated during CIU. The comparison of CIU fingerprints (IM drift times as a function 1215 of accelerating voltages) obtained for $\mathrm{P}$ and different $\mathrm{P}: \mathrm{L}$ systems can help to differentiate 1216 conformational populations of very close CCS (<1\%, [217]). Ruotolo first showed that CIU 1217 experiments allow differentiation of the effects of ligand binding not normally observed by MS 1218 alone on wild-type (wt) and disease-associated variants of tetrameric transthyretin (TTR) [218]. 1219 CIU experiments were able to show that the natural thyroxine ligand of TTR stabilizes wt TTR 1220 but also disease-associated TTR variants. Similar experiments were reported for ligand 1221 binding on model proteins like FK-binding protein, lysozyme or myoglobin [219]. More recently, 1222 CIU experiments have been reported for the identification of kinase inhibitors that specifically 1223 bind to the inactive conformation of the protein. CIU patterns allowed distinguishing between 1224 inhibitors with similar $K_{D}$ values, suggesting the use of IMS-based CIU fingerprints as a new assay capable of both rapidly detecting inhibitor binding and classifying the resultant kinase binding modes. Since then CIU experiments have been reported in advanced drug discovery workflow [220], highlighting the stabilizing role of lipids in the context of membrane protein 1228 complexes [221] or the cooperative stabilization effects upon sugar binding in the concanavalin 1229 A tetramer [222]. CIU appears as a method allowing to "resolve the unresolved through gas1230 phase measurements" [213]. 
7.4.3. Ion mobility spectrometry for membrane proteins

1233 IM-MS is also a well-established strategy to study the global conformation of membrane 1234 proteins and the characterization of their interactions with ligand/lipids [223-225]. Membrane 1235 proteins are involved in many vital cellular processes, such as trafficking and signal 1236 transduction [226,227]. Due to the high hydrophobicity associated to their structure, membrane 1237 proteins are transferred into the mass spectrometer encapsulated within detergent micelles to 1238 preserve their native-like structure [167]. Detergent aggregates strongly suppress ionization in 1239 ESI and need to be removed to perform MS analysis. Therefore, ionization source conditions 1240 such as pressure, temperature, and acceleration voltages are critical for membrane protein 1241 analysis. The protective role of detergent aggregates was clearly showed in the case of the 1242 transmembrane complex BtuC2D2 [223]. After increasing the number of collisions to produce 1243 the release of the complex from the micelle, the intact tetramer can be observed. The same 1244 ion channel (BtuC2D2) and a transporter (KirBac3.1) were characterized by Robinson and 1245 coworkers [224] to compare the average CCS of both tetramers after release from the 1246 detergent micelles. Different activation energies were applied and the overall size of both 1247 tetramer remained constant. The same experiment was performed upon activation of their 1248 respective trimers. In this case, KirBac3.1 trimer exhibited one single distribution that remained 1249 constant through ion activation, however, a bimodal distribution was observed for the BtuC2D2 1250 trimer when the acceleration voltage was set to $240 \mathrm{~V}$ being consistent with two conformer 1251 populations. Experimental CCS of BtuC2D2 and KirBac3.1 trimers were smaller compared to 1252 the theoretical ones, suggesting that trimer structures collapsed after the removal of one 1253 subunit.

1254 CCS measurements played a critical role in the modeling strategy of full length OmpA (FL1255 OmpA) dimer structure [167]. Native MS data showed monomeric and dimeric populations 1256 while further IM-MS experiments revealed compact dimers and extended and compact 1257 monomers. The same experiment was carried out for the N-terminal domain (TM-OmpA), 1258 where only the monomer structure could be observed. These results combined with native MS 
confirmed that dimer interface was located in the C-terminal 188-276 amino acid sequence. A

1260 low-resolution structure for the FL-OmpA dimer assembly was proposed based on IM-MS data 1261 as well as cross-linking constraints. The theoretical model of the FL-OmpA dimer is sustained by the good agreement between experimental and theoretical CCS.

1263 Amphipatic polymers (APols) have been shown to be a suitable alternative to detergent 1264 aggregates for preserving membrane protein water solubility [228]. Watkinson et al. studied 1265 the influence of several APols on the structure and activity of three outer membrane proteins 1266 (Omp), namely PagP, OmpT, and tOmpA [229]. The catalytic activity of these three Omp 1267 strongly varies as a consequence of amphipols interactions, despite the fact that circular dichroism data indicate that PagP, OmpT, and tOmpA native structures is preserved in the amphipols. The structures of the released Omp were assessed by ESI-IMS-MS. The experimental CCS of the lower charge states were in good agreement with theoretical CCS 1271 from protein data bank (PDB)-coordinates indicating that APols tested in this study do not 1272 induce any significant structural changes.

1273 Membrane proteins have shown strong interactions with lipids $[167,230]$. They play an 1274 essential role in membrane protein structure, folding, and activity $[230,231]$. Laganowsky and coworkers studied the selectivity of lipid binding to membrane proteins by using native MS and IMS. The stability of the mechanosensitive channel of large conductance (MscL), aquaporin Z 1277 (AqpZ) and the ammonia channel (AmtB) was assessed upon lipid binding by monitoring the 1278 unfolding process of the membrane proteins as a function of collision voltage (CIU 1279 experiments). CIU fingerprints revealed that upon the addition of CDL and PG, the stabilisation 1280 energy of AmtB dramatically increased. This result is based on the increase of the collision 1281 voltage required to induce unfolding process of the lipid complexes compared to the apo structures. In the case of AqpZ and MscL, the same stabilization effect was observed after the 1283 addition of the different lipids. These results emphasize the interest on CIU experiments to 1284 study the selectivity of membrane proteins toward lipids, and how this interaction can modulate 1285 their function. In this case, the trap cell was used for releasing membrane proteins from 1286 detergent micelles as well as to induce protein unfolding. To avoid the presence of detergent 
1287 aggregates in the trap cell, "in-source" fragmentation was performed by increasing the source 1288 temperature up to $120 \mathrm{~V}$ [225] (Figure 10), thus detergent aggregates are removed before the 1289 entrance of the ions in the trap cell. Ions are subsequently activated (CIU experiments) with or 1290 without previous ion selection (IMS-MS or MS-IMS-MS respectively) to monitor the unfolding 1291 process of membrane proteins as a function of the activation energy. Stabilization of AmtB 1292 upon lipid binding could be evidenced by comparing the transitions of the CIU profiles. The "in1293 source" fragmentation allowed performing MS-IMS-MS CIU profiles to evaluate the 1294 stabilization energy provided upon lipid binding.

1295 Overall, for all these works, experimental CCS for membrane proteins were measured through 1296 the use of well-defined IM calibration protocols [232,233]. However, membrane protein ions 1297 exhibit lower mobility compared to soluble proteins with similar molecular weights [223] as a 1298 result of their low charge state. In a recent paper from Allison et al., it was reported that the 1299 average mobility of membrane proteins was about $30 \%$ lower than soluble proteins used for 1300 TWIMS calibration [190]. Similar native-like membrane protein standards should be used to 1301 provide an accurate CCS measurement. Due to the lack of available membrane protein standards, they suggest to use larger calibrants for reducing the error associated with the longrange extrapolation of the calibration function.

7.5. Ion mobility spectrometry for large protein assemblies and its integration to 1306 structural biology programs

1307 For protein analysis, IM-MS is mostly used to characterize conformational states or to monitor 1308 conformational changes induced upon any modification of the protein environment 1309 (experimental conditions, oligomerization, ligand binding, RNA/DNA binding, protein partner 1310 binding, etc. [41]). As the number of publications reporting the use of IM-MS for protein 1311 characterization has tremendously increased in the past 10 years, it is no more possible to 1312 draw an exhaustive picture of the possibilities of IM-MS for protein conformational 1313 characterization. IM-MS can be used to determine changes in mobility and thus conformation 1314 and CCS, properties such as conformational dynamics, folding and unfolding intermediates, 
1315 ligand-induced conformational changes, aggregation intermediates and quaternary structures

1316 (topology) $[2,28,41]$. We thus tried to focus on some relevant works performed over the past

131710 years that could serve as case studies.

1318 Native MS has been particularly useful to study the dynamics of assembly or disassembly of 1319 several macromolecular machineries, giving direct access to the stoichiometry of intermediates 1320 of assembly/disassembly through accurate mass measurement. When combined with IMS, 1321 native IM-MS enables monitoring of global conformational changes occurring during the 1322 assembly/disassembly process through accurate CCS measurements. Plots representing the 1323 CCS as a function of mass are often used to highlight assembly/disassembly events. In such 1324 representation, CCS is expected to follow a linear increase as a function of the compound 1325 mass, thus events that happen at constant CCS further suggest a major conformational 1326 change. An example of such IM-MS application is given by Van Duijn et al [234], who reported 1327 the use of native MS in combination to IMS for the study of the E. coli Cascade complex (405 $1328 \mathrm{kDa})$ and the $P$. aeruginosa Csy CRISP-complex (350 kDa) immune systems. They monitored 1329 Cascade complex disassembly either under gas-phase or in-solution dissociation experiments 1330 using native IM-MS. The results allowed to create subunit connectivity models of both the 1331 Cascade and Csy CRISPR immune systems. They compared experimentally measured $1332 \mathrm{CCS}_{\mathrm{EXP}}$ of Cascade sub-modules with values calculated using molecular modeling on the basis 1333 of the cryo-EM structure of Cascade. Finally, the interaction between Cascade and its target 1334 ssDNA was next monitored by IM-MS, highlighting a small but significant structural change for 1335 Cascade upon its association to ssDNA as evidenced by the measured $\operatorname{CCS}_{\mathrm{EXP}}\left(3 \mathrm{~nm}^{2}\right.$ 1336 experimental compared to $9 \mathrm{~nm}^{2}$ expected from calculations), which can only be explained by 1337 an altered conformation of the complex.

1338 Several case studies based on structural information derived from IM-MS to build three1339 dimensional models have been reported, including the analysis of the full clamp loader 1340 complex ( $ү 3 \delta \delta ' \Psi X, 254 \mathrm{kDa})$ in interaction with a single-stranded DNA (ssDNA) binding protein 1341 (SSB4) [235] (Figure 11), the analysis of the trp RNA binding attenuation protein (TRAP) [41], 
1342 the study of ribonucleoprotein particles (sno/sRNPs) [236] or to decipher the conformation and

1343 assembly of viruses with molecular weights ranging from 1.8 MDa up to $18 \mathrm{MDa}$ [237-239].

1344 Most of these studies were performed on TWIMS instruments. However, the group of 1345 Fernandez-Lima recently reported for the first time the use of TIMS instruments for accurate 1346 CCS measurements on protein complexes [240]. As CCS values alone give no detailed 1347 information on subunit structure within the complex, IM-MS analyses can be coupled to gas1348 phase activation techniques including collision induced dissociation (CID) and more recently 1349 surface induced dissociation (SID) to generate sub-complexes, SID being more adapted to 1350 retain native-like structures in the gas phase [241,242]. In addition, crosslinking strategies have 1351 been proven to be valuable to capture transient complex, protein complexes modified with 1352 either charged crosslinkers or tags, but not those treated with neutral crosslinkers, exhibiting 1353 enhanced stability in the gas phase relative to CIU [243].

1354 Overall, these studies of large heteromeric complexes analyzed by IM-MS, coupled with 1355 integrative modelling, highlight the potential of such an approach to reveal structural features 1356 of previously unknown complexes of high biological importance.

1357 IM-MS can be considered today as a complementary low-resolution technique for structural 1358 biology. Indeed, hybrid approaches in structural biology have gained considerable interest for 1359 uncovering the molecular architectures of large and transient biological systems. MS-based 1360 methods, and in particular IM-MS, and structural electron microscopy can complement 1361 conventional tools, such as X-ray crystallography and NMR spectroscopy [244,245]. Today, 1362 IM-MS is often applied to the structural elucidation of protein assemblies that have failed high1363 throughput crystallization or NMR spectroscopy screens. In the interpretation of the IM data 1364 for multiprotein complexes, there are two main steps to interpret IM for multiprotein complexes: 1365 the conversion of drift times into CCS values, and their comparison with model protein 1366 architectures.

1367 Such IM-MS and MS/MS measurements are often integrated with other sources of structural 1368 information to build comprehensive models of intact multiprotein complexes that remain 
1369 refractory to more classical structural biology techniques. For instance, CCS restraints from 1370 IM-MS have been successfully combined to distance constraints from chemical crosslinking 1371 approaches to determine the best model among hundreds or thousands of computationally 1372 generated models for the study of the proteasomal base [246] or for the outer membrane 1373 protein A from Escherichia coli [247]. Combining IM-MS to solution hydrogen/deuterium 1374 exchange MS (HDX-MS) seems also appealing and has been reported for the characterization 1375 of flexible serpins [248] or on the Kai circandian complex [249]. For this latter system, native 1376 MS revealed that the complex formed between two of the components (KaiB and KaiC) is a 1377 dodecamer; HDX-MS provided their binding interface and finally the structural restraints 1378 derived from HDX were then combined with IM-derived CCSs and used as input for the 1379 HADDOCK web server to generate models of the KaiC6B6 complex. IM-MS is an emergent 1380 technology for structural proteomics, capable of assessing multiprotein topologies from 1381 complex mixtures using minute amounts of sample. Computational analysis is a key aspect of 1382 IM-MS data interpretation, and developments in this area are likely to be rapid over the next 13835 years.

7.6. Biopharmaceuticals

MS performed under non-denaturing conditions (native MS) has emerged as a promising 1387 technique for mAb-related compound characterization [250,251]. As a logical evolution, 1388 hyphenation of IMS to native MS was also rapidly implemented for biopharmaceutical analysis. 1389 Since IMS provides a global view of conformational differences, it is a good choice for 1390 preliminary investigations of mAb conformations, where the shape and size of the molecule 1391 are of interest. IMS can either be used qualitatively mostly to address heterogeneity issues, 1392 but also in a quantitative way, to determine CCS values or to directly evaluate the relative 1393 amounts of the different species present in solution from IMS data. IMS can also serve to 1394 address more fundamental issues through collision induced unfolding (CIU) experiments, in 1395 which collisional heating is applied in the gas-phase to unfold the protein, which is 1396 subsequently separated by IM and then analyzed by MS. A typical CIU plot presents IM drift 
1397 times as a function of increasing collisional energies applied in the region in front of the IM cell

1398 [252]. CIU fingerprints represent the amount of protein unfolding as a function of collisional 1399 energy applied. Those unfolding patterns have proven to be highly sensitive to small variations 1400 in protein structure that cannot be easily resolved by IMS alone. Figure 12 summarizes the 1401 IMS-based information that can be obtained for biopharmaceutical characterization.

1403 IMS for disulfide bridge heterogeneity - The first application of IMS for mAb structural 1404 characterization was reported in 2010 for the rapid characterization of disulfide variants in 1405 intact IgG2 mAbs [253]. IMS revealed 2 to 3 gas-phase conformer populations for lgG2s, 1406 whereas a single gas-phase conformer was revealed for either an IgG1 or a mutated IgG2, 1407 providing strong evidence that the observed IgG2 gas-phase conformers are related to 1408 disulfide bond heterogeneity. Similarly, Jones et al. have used IMS to rapidly provide 1409 information on the higher order structure of a series of wild type and mutant lgG2 mAbs [254].

1411 IMS for global conformational characterization - Given that IM is fast and gives a global view 1412 of mAb-related compound conformation, several groups have addressed the global 1413 conformation of $\mathrm{mAb}, \mathrm{ADC}$ or bispecific antibodies compounds using IMS, attempting to 1414 attribute CCSs to given mAb isotype conformations and even to particular conformational 1415 heterogeneities. Pritchard et al. used this approach to detect three monomer conformations 1416 and two dimers for a commercial rhGH-specific antibody [255]. A quantitative analysis of the 1417 ATDs revealed that $\sim 80 \%$ of the species present in the sample was in the second monomer 1418 conformation. Pacholarz et al. reported structural differences between IgG1 and IgG4 based 1419 on IMS measurements [38]. Interestingly, the ${ }^{\mathrm{DT}} \mathrm{CCS}_{\mathrm{He}}$ values obtained on a linear drift tube 1420 IM instrument in helium (61.1-83.2 $\left.\mathrm{nm}^{2}\right)$ were in agreement with those measured on TWIMS 1421 instruments by other groups for different lgGs, which demonstrates the robustness of the CCSs 1422 calculated from properly calibrated IM-MS data. However, the experimentally IMS-estimated 1423 CCS values were shown to be significantly lower than the CCS determined either from crystal 1424 structures or from MD simulation $[38,192]$. This has recently been explained by the flexibility 
1425 of the hinge region which collapses in the gas phase, such that the measured CCS of the mAb

1426 is substantially less than both the predicted CCS from its crystal structure and the in vacuum

1427 MD simulation [192].

Real-time IMS to monitor dynamic processes - IMS has also been employed to monitor dynamic processes such as the IgG4 Fab-arm exchange that produces bispecific mAbs, 1431 constituted of two different half-antibody arms, each of which recognizes a distinct antigen, a 1432 particularly valuable property for therapeutic applications. In combination with time-resolved 1433 native MS, IMS was used to monitor the formation of the bispecific mAbs in real time [256], the 1434 CCSs of the bsAb $\left(66.9 \mathrm{~nm}^{2}\right)$ being intermediate to those of the original mAbs $(65.6$ and 69.6 $\left.1435 \mathrm{~nm}^{2}\right)$.

IMS for ADC drug conjugation heterogeneity assessment - IMS can also be of great interest 1438 for the characterization of drug conjugation heterogeneity/homogeneity for antibody-drug 1439 conjugate characterization. IMS can provide several information, among which the global 1440 conformation of all detected species including conjugated and unconjugated forms or the 1441 global conjugation profile and average drug-to-antibody-ratio (DAR) through a semi1442 quantitative interpretation of IMS data (integration of ATDs areas). Debaene et al. reported the 1443 IMS characterization of brentuximab vedotin, the reference cysteine ADC [257]. The different $1444{ }^{\mathrm{TW}}$ CCS of the multiple drug loads were calculated, evidencing slight conformational changes 1445 that occur upon payload binding. The average DAR determined from IMS was $3.7 \pm 0.1$ (vs. 14464.0 expected), consistent with values obtained from other MS methods. In addition, 1447 hydrophobic interaction chromatography (HIC) fractions were collected and analyzed 1448 individually by IMS, revealing conformational homogeneity of each drug load species. Marcoux 1449 et al. next performed a similar study on trastuzumab emtansine (T-DM1), the reference lysine 1450 ADC already on the market [258]. The detailed structural picture provided by IM-MS was 1451 complemented by molecular modeling. As for the cysteine compound, native IMS highlights 1452 the increase in heterogeneity that occurs when trastuzumab is conjugated with DM1. The 
1453 resulting drug distribution profile and average DAR $(3.4 \pm 0.2)$ are also consistent with values

1454 obtained from other MS methods (average DAR of 3.5). Recently, extensive conformational 1455 characterization by IMS of a new generation site-specific ADC enabled detection of different 1456 IM drift times for unconjugated mAb and the DAR4 site-specific ADC [259]. Again, average 1457 DAR values along with drug load distributions could be estimated. Altogether, these studies 1458 demonstrate the capabilities of native IMS to directly access site-specific ADC conformational 1459 information through the measurement of IM drift times and CCS calculations.

1461 Qualitative IMS for comparability studies - IMS is a forefront methodology for qualitative batch 1462 to batch comparison. In case of comparability studies between originators and biosimilars 1463 (copy versions of mAb), IMS can address global conformational identity. IMS has been 1464 employed to highlight conformational heterogeneity in a biosimilar version of trastuzumab. 1465 Different IM drift times were observed for trastuzumab and its biosimilar version, which could 1466 be attributed to the presence of additional species bearing 0 or 1 glycan per Fc on the biosimilar 1467 version [260]. Similarly, a combination of MS-based approaches, including native MS and IMS 1468 were used to compare the originator compound Remicade (infliximab, Janssen) to its biosimilar 1469 product Remsima (Celltrion) [261]. Native IMS revealed similar IM drift times for mAb 1470 fragments, monomers, and dimers in both sample. Extensive comparative studies of 1471 monoclonal antibody drug products representing different IgG subclasses, manufacturers, and 1472 lots were also reported by Fergusson et al. [262] using a set of IMS-based metrics to assess 1473 reproducibility and robustness issues of IMS. IMS enabled detection of differences between 1474 monoclonal antibodies with the same target protein but different production techniques, as well 1475 as products with different targets. All of these studies conclude that native IMS analysis 1476 presents the advantage to require only a minimal (when manual buffer exchange is used) or 1477 no (when size exclusion is coupled to IMS using volatile salts) sample preparation, along with 1478 a global conformational characterization of the studied mAb-related compounds. 
CIU experiments and variable-temperature IMS to study mAb unfolding patterns - IMS

1481 methodologies based on CIU experiments have been reported to address rapid conformational 1482 characterization of mAbs [263]. CIU experiments provide specific unfolding fingerprints that 1483 can be used to distinguish similar conformations, for instance mAb isotypes (IgG1 vs IgG2 vs 1484 lgG3 vs IgG4) with different numbers and/or patterns of disulfide bonding and levels of 1485 glycosylation. CIU experiments have also been reported for site-specific ADC characterization 1486 [259]. Even if different IM drift times were obtained for the ADC and its unconjugated mAb 1487 counterpart, they both exhibited similar intact CCS, which did not allow distinction between a 1488 mass effect or a real conformational change induced upon drug conjugation. CIU experiments 1489 were thus performed on both ADC and its unconjugated mAb version. Different CIU unfolding 1490 patterns were obtained for the unconjugated mAb compared to the site-specific DAR4 ADC, 1491 revealing increased ADC stability towards unfolding as compared with the unconjugated mAb. 1492 Here, CIU experiments allowed circumventing the lack of IM resolution through the 1493 establishment of unfolding patterns. CIU experiments were also used for comparability studies 1494 between biosimilar and originator [261]. Pisupati et al. compared the CIU patterns of the 1495 originator Remicade and its biosimilar version Remsina. Both compounds revealed remarkably 1496 similar patterns. While CIU patterns were globally similar (two unfolded states at nearly 1497 identical voltages for each CIU transition), a marked difference in the variance associated with 1498 the CIU fingerprint for Remsima at low collision voltages was observed. CIU experiments can 1499 thus be used for batch-to-batch comparison or more generally for comparability studies. Due 1500 to its higher reproducibility compared to traditional collision cross section IMS studies, CIU 1501 experiments remain a promising technique for advanced analytical characterization of protein 1502 therapeutics [262].

1503 Variable Temperature IMS (VT-IM-MS) were also reported to address mAb conformation and 1504 conformational transitions induced upon IM cell temperature variations [264]. In this work, the 1505 thermal stability of three types of intact $\lg G$ molecules (with the same antigen binding 1506 specificity) has been probed by variable-temperature IM-MS. In these experiments, mass 1507 spectra and IM plots were acquired, while the temperature of the helium gas in the drift tube 
1508 was increased (from 300 to $550 \mathrm{~K}$ ). While the native mass spectra showed no significant

1509 change as the temperature was increased (other than improved desolvation), the ${ }^{\mathrm{DT}} \mathrm{CCSS}_{\mathrm{He}}$ of 1510 the $\lg \mathrm{G} 1$ and $\lg \mathrm{G} 4$ isotypes decreased because of compaction. The maximum collapse 1511 occurred at a higher temperature for $\lg \mathrm{G} 1(400 \mathrm{~K})$ than for $\lg \mathrm{G} 4(360 \mathrm{~K})$, in agreement with 1512 solution-phase studies in which $\operatorname{lgG} 1$ was found to be more thermally stable than $\operatorname{lgG} 4$. 1513 Differences in the extent of unfolding (an increase and widening of ${ }^{\mathrm{DT}} \mathrm{CCS}_{\mathrm{He}}$ ) among the $\operatorname{lgG}$ 1514 subclasses were also observed at high buffer gas temperatures, IgG4 unfolding at a lower 1515 temperature and to a greater extent than IgG1. VT-IM-MS provides insights into mAbs 1516 structural thermodynamics and can be considered as a promising tool for thermal-stability 1517 studies for proteins of therapeutic interest.

1518

1519 In conclusion, IMS is an interesting methodology for the characterization of intact antibodies 1520 and related formats (bispecific, ADCs) and may be useful to routinely fingerprint higher order 1521 structures.

1522 Reproducibility of IMS measurements and mobilograms, as well as the suitability of IMS to 1523 differentiate between and/or characterize different monoclonal antibody drug products has 1524 been evaluated by the FDA [262], evidencing that the use of summed charge state datasets 1525 and the analysis of metrics beyond drift time should be preferred for a more comprehensive 1526 comparative study between different monoclonal antibody drug products. Also the added value 1527 of CIU experiments, that have proved to be more reproducible than classical IMS 1528 measurements (IM drift times and CCS), offers new possibilities for mAb-related compound 1529 conformational characterization. IMS measurements can finally be extended to investigate 1530 conformational changes induced upon antigen binding to individual $\mathrm{mAb}$ isoforms in order to 1531 gain more structural insights.

7.7. Oligonucleotides

1534 IM-MS applied to oligonucleotides has been mainly used to gain conformational insights of 1535 such highly polymorphic macromolecule. Indeed, besides the classical and widely known 
1536 double-stranded DNA sequence (duplex), a plethora of other helical structures can be found, 1537 such as hairpin, pseudoknot, triplex, cruciform (Holliday junction) and G-quadruplex structures. 1538 Most of these DNA conformations have been studied by IM-MS and molecular dynamics, 1539 highlighting a more dynamic and complex behaviour in the gas-phase as compared to proteins 1540 [265].

1541 One of the first research article was published by Clemmer et al. [266]. They reported a 1542 conformational study of an oligonucleotide comprising ten thymine nucleotides $\left(T_{10}\right)$. The 1543 authors highlighted that the gas-phase conformations observed by IM-MS were strongly 1544 dependent of the charge states: lower charge states corresponded to globular conformers 1545 (lower CCS) and higher charge states to elongated forms (higher CCS). Moreover, by 1546 comparing the experimental $\operatorname{CCS}\left(\Omega_{\mathrm{EXP}}\right)$ with calculated $\operatorname{CCS}\left(\Omega_{\mathrm{THE}}\right)$ of conformers generated 1547 by modelling technique (by using one of the first parametrisation of EHSS model), [48] a strong 1548 dependency was found between the gas-phase conformations and the number and especially 1549 the locations of charged sites. It should be noted that charge states observed by IM-MS reflect 1550 the extension of the neutralization of the ionizable groups. Whereas in proteins, the ionizable 1551 groups are represented by the $\mathrm{N}$ - and $\mathrm{C}$-terminal residues plus the basic and acidic side 1552 chains, in nucleic acids they are represented by the phosphodiester linkages that are all 1553 potentially and equally ionizable. In the case of $T_{10}$, neutralization could occur along the nine 1554 phosphate groups with a number of combination equal to $9 ! /[n !(9-n) !]$, thus several protomers 1555 could arise for each unique charge state. Authors calculated theoretical CCS $\left(\Omega_{T H E}\right)$ for several 1556 different charge site assignments and they found that conformers having the same charge 1557 state $(-5)$ showed a compact conformation $\left(\Omega_{\text {THE }}=501 \pm 19 \AA\right.$ ) when having close located 1558 charges, and a most open conformation $\left(\Omega_{\mathrm{THE}}=608 \pm 21 \AA\right.$ ) when charges were more evenly 1559 distributed throughout the oligomer. Open conformation was found to match better with the 1560 experimental values $\left(\Omega_{\operatorname{EXP}}=627 \pm 6 \AA\right)$ and thus it was accordingly assigned [266]. Although 1561 the authors themselves questioned the absolute accuracy of the calculations, the concepts 1562 highlighted on number and locations of charged sites affecting the conformers shape were 
1563 fundamental to understand how different from solution nucleic acids might behave in the gas1564 phase.

1565 Duplex stability and its behaviour in gas-phase has also been investigated by IM-MS and 1566 molecular modelling; initial contributions in this direction came from Bowers group [267-269]. 1567 First studies involving $d(C G)_{n} \cdot d(C G)_{n}$ duplexes [267] demonstrated that a globular form is the 1568 dominant conformer for up to 8-mer length oligomer, whereas for longer duplexes only the 1569 helical structure was observed, keeping quite good memory of the solution structure. Then, the 1570 effect of the oligomer sequence composition on the overall conformation of the duplex was 1571 investigated by studying a series of 14-mer duplexes containing both $C \cdot G$ and $A \cdot T$ pairs in 1572 different sequences [268,269]. It turns out that the A.T pairs were preferentially broken over 1573 C.G pairs and they appear more easily disrupted when placed at the ends of the duplex, while 1574 forming bubble if placed at the middle of the sequence. Therefore, overall results showed that 1575 duplex helicity was usually maintained in the gas-phase on the ms timescale but the structures 1576 stability was dependent of the length of the oligomer, its bases composition and its sequence 1577 order. However, as highlighted in [270], the structural comparison between the duplex native 1578 forms in solution and the conformations assumed in the gas-phase remained questionable, since the experiments were performed in non-native solution conditions. Indeed, a more recent 1580 contribution on duplex nucleic acids behaviour in the gas-phase upon native electrospray mass 1581 spectrometry came from the Orozco and Gabelica groups [270]. A compelling study on short 1582 (12-bp) and long (36-bp) DNA and RNA duplexes was performed by ESI-DTIM-MS, and a 1583 significant compaction of the duplex nucleic acids was observed for ions sprayed from native 1584 solution conditions. $\Omega_{\text {THE }}$ of the structures adopted in solution were obtained upon MD 1585 simulation carried out in water and then compared to $\Omega_{\mathrm{EXP}}$ ( ${ }^{\mathrm{DT}} \mathrm{CCS} \mathrm{S}_{\mathrm{He}}$ ) that were found 1586 significantly lower than the predicted values. To investigate the behaviour of the duplexes in 1587 the gas-phase, an extensive molecular modelling study was performed, including DFT and 1588 semi-empirical calculations for small helices and short duplexes plus MD simulations for long 1589 duplexes. Finally, authors were able to demonstrate that the compaction experimentally 1590 observed by ESI-IM-MS was related to the self-solvation of the phosphates groups occurring 
1591 through new phosphate-phosphate interactions that undermine the Coulomb-driven expansion 1592 of the duplexes. These metastable conformations arose in part during the desolvation process 1593 and rearranged by self-solvation while in the gas-phase, thus resulting significantly compacted 1594 compared to the starting structures [270]. Similarly, Devine et al. described the gas-phase 1595 compaction of two 35-mer RNAs having similar mass, but different sequences and secondary 1596 structures [192]. Reference $\Omega_{\text {THE }}$ values of the two 35-mer RNAs were obtained from an NMR 1597 and a crystal structure, respectively. Nevertheless, ESI-TWIMS-MS of the RNAs yielded to 1598 identical $\Omega_{\mathrm{EXP}}\left({ }^{\mathrm{TW}} \mathrm{CCS}_{\mathrm{N} 2}\right.$ ) that were significantly lower than the predicted values, hence 1599 accounting for a severe compaction of the RNAs in the gas-phase. However, beyond 1600 mentioning the collapse of the RNAs, authors did not investigate further on the RNA behaviour 1601 in the gas-phase by molecular modelling studies.

1602 A quite different gas-phase behaviour was observed for higher-ordered oligonucleotide 1603 structures, namely triplex and G-quadruplex structures. In [271] $\Omega_{\text {THE }}$ obtained upon an 1604 extensive (90 $\mu \mathrm{s})$ MD simulation were compared with $\Omega_{\mathrm{EXP}}\left({ }^{\mathrm{DT}} \mathrm{CC} \mathrm{S}_{\mathrm{He}}\right.$ ) obtained by ESI-DTIM1605 MS experiments, to validate the theoretical data and assess the stability and the structure of a 1606 triplex in the gas-phase A very good agreement was found between theoretical and 1607 experimentally derived CCS. A 12-mer and 18-mer triplexes were used for the study and the 1608 overall results suggested that the gas-phase structures maintained an excellent memory of the 1609 solution structures, together with a well-preserved helicity, even though a significant number 1610 of structural alterations were observed. Indeed, a sligh compaction of the triplex (in respect to 1611 the solution structure) was observed for the most abundant charge states, but no triplex 1612 disruption was observed in any case.

1613 Even a higher DNA structure stability in the gas-phase was observed for the G-quadruplex 1614 structures that were monitored by both ESI-IM-MS and molecular modelling [272-275]. G1615 quadruplexes are characterised by the formation of G-tetrads (four planar guanines held 1616 together by Hoogsteen hydrogen bonds) that stack on top of each other to form rigid and well 1617 organised structures, thanks also to the presence of cations placed in-between the G-tetrads 1618 that further stabilise the structure. These particular DNA conformations can be formed, starting 
1619 from G-rich oligonucleotides, they can be intra-, bi-, or tetra-molecular, and they are related to 1620 cellular ageing and transformations. Evidences of G-quadruplex structure preservation in the 1621 gas-phase were reported by several groups $[276,277]$ and mainly related to the preservation 1622 of the inner cations in-between the G-tetrads [278]. Moreover, MD simulation $(0.5-1 \mu \mathrm{s})$ 1623 studies validated this preservation and proved the ability of the G-quadruplexes to keep an 1624 excellent memory of their solution structure [279], with a general excellent agreement between 1625 theoretical and experimentally derived CCS [39,268,272]. Then, the stability of G1626 quadruplexes in the gas-phase was furthermore validated after testing a series of potential 1627 ligands that were found to form stable complexes maintained also upon transfer in the gas1628 phase [280].

\section{The future of ion mobility spectrometry}

8.1. Some potential solutions to improve ion mobility resolution

1632 One of the major constraints of IMS is the limited resolution of commercial instruments. This 1633 low separation power obviously limits the applicability of IMS, as it remains difficult to 1634 differentiate structurally related compounds (species having mobilities differences of less than $1-2 \%$ are hardly distinguishable today [215]). Besides resolving isobaric compounds, a higher resolving power could also contribute to increasing the dynamic range of the measurements 1637 [8].

1638 To circumvent this limitation, there are many IMS prototypes that have been proposed by 1639 academic laboratories and providers of MS instrumentation, which are well described in the 1640 recent review of McLean and May [9]. However, it is important to keep in mind that achieving 1641 higher resolution comes at the expense of mobility range and/or sensitivity reduction. In this section, only the technical solutions that have the potential to be transformed into commercial 1643 products, at least from our point of view, will be described. Indeed, we have to keep in mind 1644 that the speed of translating IM-MS prototypes into commercial instruments in the last 5 years 1645 has increased dramatically. 
1646 As described in the first sections of this review, the DTIMS resolution is directly proportional to

1647 the square root of drift tube length and applied electric field. Therefore, there have been a lot 1648 of attempts to increase these parameters in DTIMS. However, when increasing drift tube length, the transmission may be drastically reduced (ion losses), or only a very narrow mobility range could be investigated, thus limiting the molecular coverage. In addition, a drift tube length of several meters, as described by Kemper [281] may be impractical for common laboratory

1652 space and requires very high voltages. To avoid the use of high voltages, the TWIMS 1653 technology was found to be more adapted in combination with long IMS cell. Recently, the 1654 group of Smith et al. have constructed a $13 \mathrm{~m}$ long serpentine TWIMS drift path, including 44 "U" turns to limit the required laboratory space [73]. In addition, the $13 \mathrm{~m}$ tube was included in a structure for lossless ion transmission (SLIM), to assist in ion transmission and manipulation,

1657 thus enabling a very limited loss in sensitivity. They demonstrated a baseline resolution for two 1658 isomeric sugars and also employed this new device for resolving peptides of an albumin tryptic 1659 digest. According to the authors, the resolution was increased by a factor of 9 , in comparison 1660 with commercial DTIMS instrument having a tube length of $80 \mathrm{~cm}$.

1661 Another promising strategy to improve the current resolving power of IMS may be the use of a 1662 cyclic TWIMS design to limit laboratory space. Using this approach, very high resolution can 1663 be achieved after an important number of cycles (up to 12) on a circular TWIMS drift tube of 1664 around 1 meter [9]. One of the major limitations of the circular drift tube geometry may be 1665 related to the fact that the ions of high mobility of the next cycle can lap over the ions of low 1666 mobility from the previous cycle. Since the TWIMS operates in a time dispersive manner, this 1667 point is less critical, but one of the main limitations of this design remains that the measurable mobility range decreases with every cycle needed to improve the resolution.

1669 Another strategy to improve IMS resolution is to modify the gas nature since the latter could 1670 strongly impact the separation power in FAIMS. In particular, Shvartsburg and co-workers have 1671 improved FAIMS measurements by using non-traditional buffer gases (mixtures of $\mathrm{He} / \mathrm{N}_{2}$ or $1672 \mathrm{H}_{2} / \mathrm{N}_{2}$ ). They showed that when helium rich-gases and elevated electric fields were used, the 1673 resolution was significantly increased, allowing the separation of previously unresolved 
1674 conformers of a proteolytic digest [282,283]. To avoid the electrical breakdown which is 1675 inevitable when a high proportion of $\mathrm{He}$ is used, mixtures of $\mathrm{N}_{2}$ and up to $90 \% \mathrm{H}_{2}$ were also 1676 successfully employed [284]. Some other authors have also demonstrated that drift gas with 1677 high polarizability (carbon dioxide or argon) allow achieving better resolution and peak capacity 1678 in DTIMS and TWIMS, due to the extended interactions that take place $[81,285]$. As example, 1679 Eberlin et al. show a separation of disaccharide isomers in TWIMS with carbon dioxide, while 1680 it was not possible with nitrogen [286].

8.2. Improving orthogonality between ion mobility and mass spectrometry Besides its limited resolving power, another important constraint of IMS is the lack of orthogonality with MS, since the shape of molecules in a gas phase (IMS separation criteria) is strongly correlated to its mass (MS separation criteria). In this sense, FAIMS offers a better orthogonality to MS, due to a different operation principle, in comparison with DTIMS, TWIMS and TIMS. Some authors have clearly demonstrated that the gas nature in IMS could also impact selectivity, in addition to the improvement of resolving power discussed in section 5.1 . The most remarkable example to date has been reported by Hill et al. who demonstrated the separation of enantiomers in DTIMS, by doping the drift gas with a chiral selector (2-butanol) [287]. Some other research groups have also tested different drift gases (i.e. nitrogen, helium, argon, methane) in DTIMS for separating peptides from a tryptic digest, but they showed that selectivity was less affected by the gas nature, when the ions belong to the same biomolecular 1694 class [288].

In FAIMS, the selectivity could be drastically modified when adding different polar modifiers (alcohols, acetonitrile, acetone...) to the drift gas (nitrogen) [289,290]. This was well illustrated by Varesio et al. who considered a mixture of eight pharmaceutical compounds having all a mass of $316 \mathrm{Da}$, as a case study. As illustrated in Figure 13, with pure nitrogen as transport gas, the resolving power for the eight compounds with the same nominal mass was close to 1700 zero and the eight analytes experienced a relatively similar differential mobility in the $10 \mathrm{~V}$ 1701 compensation voltage window [291]. When adding only $1.5 \%$ organic modifier, the separation 
1702 was drastically improved, particularly with isopropanol. The separation enhancement was 1703 probably due to the formation of transient clusters between the analytes of interest and the 1704 polar modifier molecules. In this example, the overall IMS separation was quite good, despite 1705 the fact that the drugs possess the same masses, because their chemical properties were 1706 different as well as their interactions with the modifiers. In another study [292], 30 drugs of 1707 abuse were investigated in FAIMS using various modifiers (i.e. acetone, acetonitrile and 1708 methanol) in the drift gas. The best IMS separation was achieved with $1.5 \%$ methanol, but this 1709 example also demonstrates the limited resolving power of FAIMS (the maximum peak capacity 1710 was less than 20). Despite the poor peak capacity, some isomeric metabolites such as 1711 hydroxylated or desmethylated ones were partially resolved, and glucuronide metabolites were 1712 differentiated from the parent drug, preventing the overestimation of the latter in case of in1713 source fragmentation. Finally, Chen et al. have also used FAIMS for the detection and 1714 quantification of nine structurally related amphetamine-type stimulants in urine in presence of 1715 dopants (i.e. $\mathrm{MeOH}$, propanol, butanol, hexanol, heptanol) and various transport gas (i.e. 1716 nitrogen, helium, argon, carbon dioxide) [293]. The authors concluded that the combination of $1717 \mathrm{He}$ and $\mathrm{MeOH}$ allows achieving the best sensitivity and selectivity for the investigated mixture 1718 of compounds.

1719 All the above mentioned studies illustrate the potential of using alternative drift gas natures or 1720 adding modifiers to the drift gas in IMS and DMS to tune selectivity. It is also clear that there 1721 are further combinations of gas and additives that could be explored to improve orthogonality 1722 between IMS and MS and some additional studies in this particular field will be helpful to make 1723 progress. However, it is also important to keep in mind that some of the current instruments 1724 on the market are not compatible with a variety of gases and modifiers and can only be used 1725 with pure nitrogen as drift gas (i.e. Waters VION, Bruker TIMS...). dimensional analytical approach) 
1729 Because the samples to be investigated in the -omic field (i.e. lipidomics, proteomics or 1730 metabolomics), in natural products analysis or in biopharmaceuticals characterization are 1731 extremely complex, there is a need to develop analytical strategies able to separate the highest 1732 number of compounds. On the chromatographic side, comprehensive 2D-LC strategy has 1733 emerged over the last few years, as a promising (but complex) alternative to very high1734 resolution 1D separation [294-299]. Beyond separation techniques, IMS and HRMS become 1735 ever popular and powerful, particularly for untargeted analysis of complex samples.

1736 To simultaneously take advantage of these chromatographic and mass spectrometric 1737 advances, Stephan et al. [105,151] have recently combined in series 2D-LC and IMS-HRMS, 1738 enabling the characterization of the most complex samples in four dimensions. According to 1739 the authors, there are many constraints when implementing such a complex analytical setup. 1740 In particular, there is a need to improve the software for managing the LC+LC-IMS/MS setup 1741 and above all the data treatment. Nevertheless, they have successfully applied the strategy to 1742 characterize plant extract (Gingko Biloba) and achieved impressive performance, with a peak 1743 capacity of 8,700 for an analysis time of only two hours [105]. In addition, for each peak 1744 observed on the 2D-LC chromatogram, the CCS and $\mathrm{m} / \mathrm{z}$ values were also available, as 1745 illustrated in Figure 14. Stephan et al. have also applied the same strategy for the analysis of 1746 wastewater [151]. They performed the analysis of wastewater sample in "regular" LC-IMS/MS 1747 and discovered 22 different pollutants using a database containing about 500 drug-like 1748 compounds and pesticides. When using the newly developed LC+LC-IMS/MS strategy, 53 1749 different compounds were identified using the exact mass and CCS values in the database. 1750 In the future, it is expected that this type of approach will be investigated for the 1751 characterization of -omic samples, as the number of detected features could certainly be 1752 strongly enhanced when using these multiple separation dimensions. Another application 1753 could be the analytical characterization of biopharmaceuticals, including monoclonal 1754 antibodies, antibody-drug conjugates, and bispecific antibodies. The addition of IMS to the 1755 more commonly used 2D-LC-MS setup would be of particular interest because it could help to 1756 differentiate isobaric compounds that are not chromatographically separated [298]. Recently, 
1757 an innovative multidimensional analytical approach combining comprehensive on-line two

1758 dimensional chromatography that consists of hydrophobic interaction chromatography (HIC)

1759 and size exclusion chromatography (SEC) to ion mobility and mass spectrometry (IM-MS) was

1760 applied for performing analytical characterization of ADCs under non-denaturing conditions.

1761 This promising approach allows obtaining in-depth and detailed information on ADC samples

1762 that cannot be reached from HIC-UV or non-denaturing mass spectrometry alone. The all-in-

1763 one analytical strategy described here permits a deep, straightforward and rapid 1764 characterization of complex ADC samples [300].

1765

1766

8.4. Improvement of data interpretation workflows and software

1767

As underlined in the previous sections, there are currently a number of new IM-MS techniques

1768

and technologies under development with the aim of further improving the performance in

1769

terms of resolution, sensitivity and orthogonality between IMS and MS. However, one of the

remaining limitations of IM-MS instrument is often represented by the software, which requires

1771 intensive development for improving speed and user-friendliness, especially when technical 1772 possibilities continue to grow.

1773 Indeed, when performing LC-IM-MS experiments, the data are extremely complex as they are

1774 acquired on many different dimensions (i.e. retention times in LC; drift times in IMS; exact 1775 mass, MS/MS spectra and signal intensity in MS). Not all softwares are well developed for 41776 dimensional (retention time, drift time, $\mathrm{m} / \mathrm{z}$, abundance) alignment of molecular features 1777 (entities) across different samples. This means that doing high-quality statistical interpretation 1778 during non-targeted (discovery) analysis can be extremely challenging or even impossible 1779 without innovation using open-source tools.

1780 In addition, files processing of large data sets may be critical. In particular, peak picking 1781 (feature finding) can be very slow and algorithms optimization is still a topic of prime interest. 1782 In this regard, Vivo-Truyols and co-workers reported the Bayesian approach for features 1783 finding in GC-HRMS [301] or GCxGC-FID [302], that could be envisaged for application in LC1784 IM-MS data treatment in the future in order to improve the peak picking process throughput. 
1785 For processing MS/MS data alongside IMS information (e.g. LC-IMS-QqTOF/MS), some 1786 solutions are proposed by the IM-MS instruments providers. For example, Waters proposes 1787 the $\mathrm{MS}^{\mathrm{E}}$ approach, which records data without discrimination or preselection, while Agilent 1788 uses an approach called "alternating frames", where two MS analyses (high energy and low energy) can be obtained within a single acquisition in order to associate MS/MS and MS

1790 spectra via drift time alignment. Such approaches can be already implemented into analytical 1791 methods, but software-based extraction of all the information together is awaiting for further 1792 developments and it will also require careful validation for non-targeted workflows.

1793 Another important area of software development regards the data deconvolution strategies, 1794 which allows the extraction of additional information from partially resolved ion mobility data, 1795 taking into account the orthogonal information obtained from ion fragmentation taking place 1796 after the ion mobility cell. This promising approach can allow the reconstruction of ion mobility 1797 spectra for individual species, especially for the challenging topic of isomer differentiation, as 1798 reported in [66].

1799

\section{Conclusion}

1801 This review paper summarizes the current status of IM-MS and main application fields where 1802 the technology has been employed to date. As discussed, the main benefits of IM-MS are the following: i) Improving the confidence in peak annotation thanks to the use of CCS values in 1804 addition to retention time, exact mass and fragment spectra, ii) Reducing the complexity of 1805 MS/MS spectra, and rapid association of fragments with their monoisotopic precursors by 1806 taking into account the mobility alignment, iii) Increasing the overall resolution of the analysis, 1807 since MS and HRMS are obviously unable to separate isobaric compounds, iv) In-depth 1808 studies of the gas phase conformation of large biomolecules such as proteins and 1809 oligonucleotides and comparison of calculated and experimental CCS values.

1810 Among the applications reported in this contribution, adding an IMS dimension looks 1811 particularly attractive in metabolomics for improving peak annotation confidence. It can also 1812 offer a higher resolving power for a few families of compounds having relatively high flexibility 
1813 and containing a substantial number of isomers. Among them, lipids and glycans are

1814 particularly well adapted to IMS and have been studied extensively during the last few years.

1815 Finally, due to the inherent complexity of protein biopharmaceuticals and oligonucleotides, 1816 there will certainly be even more interest for IMS in the coming years in order to increase the 1817 amount and quality of information that can be obtained from their analysis.

1818 As illustrated throughout this paper, the field of IMS is very active and instrumental advances 1819 are being regularly published. In this regard, one of the remaining bottlenecks of IMS is its 1820 limited resolution. Therefore, there is a need for longer IMS drift cells, or alternative IMS 1821 designs, as an order of magnitude improvement in resolving power would clearly revolutionize 1822 the field [16]. Another important limitation of the technology is the poor orthogonality between 1823 IMS and MS. To improve this aspect, the addition of dopants (small amount of organic modifier) 1824 to the IMS drift gas appears as a promising strategy, but this solution is unfortunately not 1825 compatible with all IM-MS instruments. Finally, even though the sensitivity achieved in IM-MS 1826 has been drastically improved over the last few years, there could still be some sensitivity loss 1827 when adding an IMS dimension prior to MS. To avoid, or at least limit this issue, Smith et al. 1828 have recently developed some IMS structures with lossless ion manipulations (SLIM), which 1829 seems certain to be implemented in the next IMS generation [303].

\section{The authors have declared no conflict of interest.}




\section{References}

[1] Uetrecht, C., Rose, R. J., van Duijn, E., Lorenzen, K., Heck, A. J. R., lon mobility mass spectrometry of proteins and protein assemblies. Chem. Soc. Rev. 2010, 39, 16331655.

[2] Lanucara, F., Holman, S. W., Gray, C. J., Eyers, C. E., The power of ion mobility-mass spectrometry for structural characterization and the study of conformational dynamics. Nat. Chem. 2014, 6, 281-294.

[3] Paglia, G., Astarita, G., Metabolomics and lipidomics using traveling-wave ion mobility mass spectrometry. Nat. Protoc. 2017, 12, 797-813.

[4] Lapthorn, C., Pullen, F., Chowdhry, B. Z., Ion mobility spectrometry-mass spectrometry (IMS-MS) of small molecules: separating and assigning structures to ions. Mass Spectrom. Rev. 2013, 32, 43-71.

[5] Gray, C. J. J., Thomas, B., Upton, R., Migas, L. G. G., Eyers, C. E. E., Barran, P. E. E., Flitsch, S. L. L., Applications of ion mobility mass spectrometry for high throughput, high resolution glycan analysis. Biochim. Biophys. Acta - Gen. Subj. 2016, 1860, 1688-1709.

[6] Szymańska, E., Davies, A. N., Buydens, L. M. C., Chemometrics for ion mobility spectrometry data: recent advances and future prospects. Analyst 2016, 141, 56895708 .

[7] Kanu, A. B., Dwivedi, P., Tam, M., Matz, L., Hill, H. H., Ion mobility-mass spectrometry. J. Mass Spectrom. 2008, 43, 1-22.

[8] Zheng, X., Wojcik, R., Zhang, X., Ibrahim, Y. M., Burnum-Johnson, K. E., Orton, D. J., Monroe, M. E., Moore, R. J., Smith, R. D., Baker, E. S., Coupling front-end separations, ion mobility spectrometry, and mass spectrometry for enhanced multidimensional biological and environmental analyses. Annu. Rev. Anal. Chem. 2017, 10, 71-92.

[9] May, J. C., McLean, J. A., Ion mobility-mass spectrometry: time-dispersive instrumentation. Anal. Chem. 2015, 87, 1422-1436.

[10] Cohen, M. J., Karasek, F. W., Plasma chromatography - a new dimension for gas chromatography and mass spectrometry. J. Chromatogr. Sci. 1970, 8, 330-337. 
1863 [11] May, J. C., Goodwin, C. R., Lareau, N. M., Leaptrot, K. L., Morris, C. B., Kurulugama, 1864 R. T., Mordehai, A., Klein, C., Barry, W., Darland, E., Overney, G., Imatani, K., Stafford,

[12] Stow, S. M., Causon, T., Zheng, X., Kurulugama, R. T., Mairinger, T., May, J. C., Rennie,

[13] Doneanu, C. E., Anderson, M., Williams, B. J., Lauber, M. A., Chakraborty, A., Chen, W., Enhanced detection of low-abundance host cell protein impurities in high-purity monoclonal antibodies down to $1 \mathrm{ppm}$ using ion mobility mass spectrometry coupled with multidimensional liquid chromatography. Anal. Chem. 2015, 87, 10283-10291.

[14] Giles, K., Pringle, S. D., Worthington, K. R., Little, D., Wildgoose, J. L., Bateman, R. H., Applications of a travelling wave-based radio-frequency-only stacked ring ion guide. Rapid Commun. Mass Spectrom. 2004, 18, 2401-2414.

[15] Pringle, S. D., Giles, K., Wildgoose, J. L., Williams, J. P., Slade, S. E., Thalassinos, K., Bateman, R. H., Bowers, M. T., Scrivens, J. H., An investigation of the mobility separation of some peptide and protein ions using a new hybrid quadrupole/travelling wave IMS/oa-ToF instrument. Int. J. Mass Spectrom. 2007, 261, 1-12.

[16] Ewing, M. A., Glover, M. S., Clemmer, D. E., Hybrid ion mobility and mass spectrometry 1885 as a separation tool. J. Chromatogr. A 2016, 1439, 3-25.

[17] Giles, K., Williams, J. P., Campuzano, I., Enhancements in travelling wave ion mobility resolution. Rapid Commun. Mass Spectrom. 2011, 25, 1559-1566.

[18] FAIMS/lon Mobility, https://yost.chem.ufl.edu/research/faims/ (last time accessed: July 25, 2017).

[19] Isenberg, S. L., Armistead, P. M., Glish, G. L., Optimization of peptide separations by differential ion mobility spectrometry. J. Am. Soc. Mass Spectrom. 2014, 25, 1592- 
1599.

1892 [20] Hernandez, O., Isenberg, S., Steinmetz, V., Glish, G. L., Maitre, P., Probing mobilityselected saccharide isomers: selective ion-molecule reactions and wavelength-specific IR activation. J. Phys. Chem. A 2015, 119, 6057-6064.

1895

[21] Schneider, B. B., Nazarov, E. G., Londry, F., Vouros, P., Covey, T. R., Differential 1896 mobility spectrometry/mass spectrometry history, theory, design optimization, simulations, and applications. Mass Spectrom. Rev. 2016, 35, 687-737.

1898 [22] Shvartsburg, A. A., Seim, T. A., Danielson, W. F., Norheim, R., Moore, R. J., Anderson, 1899 G. A., Smith, R. D., High-Definition Differential Ion Mobility Spectrometry with Resolving Power up to 500. J. Am. Soc. Mass Spectrom. 2013, 24, 109-114.

[23] Xuan, Y., Creese, A. J., Horner, J. A., Cooper, H. J., High-field asymmetric waveform 1902

http://www.seadm.com/products/technological-modules/separation/planar-dma-cell/ (last time accessed: July 25, 2017).

[25] Pomareda, V., Lopez-Vidal, S., Calvo, D., Pardo, A., Marco, S., A novel differential mobility analyzer as a VOC detector and multivariate techniques for identification and quantification. Analyst 2013, 138, 3512.

1911 [26] timsTOF, https://www.bruker.com/fileadmin/user_upload/8-PDFDocs/Separations_MassSpectrometry/Literature/Brochures/1844502_timsTOF_brochu re_05-2016_ebook.pdf (last time accessed: July 25, 2017).

1914 [27] Revercomb, H. E., Mason, E. A., Theory of Plasma Chromatography/Gaseous Electrophoresis- A Review. Anal. Chem. 1975, 47, 970-983.

1916 [28] Ruotolo, B. T., Benesch, J. L. P., Sandercock, A. M., Hyung, S.-J., Robinson, C. V, Ion mobility-mass spectrometry analysis of large protein complexes. Nat. Protoc. 2008, 3, $1139-1152$. 
1919 [29] Atmanene, C., Petiot-Bécard, S., Zeyer, D., Van Dorsselaer, A., Vivat Hannah, V., 1920 Sanglier-Cianférani, S., Exploring key parameters to detect subtle ligand-induced protein conformational changes using traveling wave ion mobility mass spectrometry.

1923

1924

1925

1926

1927

1928

1929

1930

1931

1932

1933

1934

1935

1936

1937

1938

1939

1940

1941

1942

1943

1944

1945

1946 Anal. Chem. 2012, 84, 4703-4710.

[30] Ouyang, H., Larriba-Andaluz, C., Oberreit, D. R., Hogan, C. J., The collision cross sections of iodide salt cluster ions in air via differential mobility analysis-mass spectrometry. J. Am. Soc. Mass Spectrom. 2013, 24, 1833-1847.

[31] Bernstein, S. L., Dupuis, N. F., Lazo, N. D., Wyttenbach, T., Condron, M. M., Bitan, G., Teplow, D. B., Shea, J.-E., Ruotolo, B. T., Robinson, C. V., Bowers, M. T., Amyloid- $\beta$ protein oligomerization and the importance of tetramers and dodecamers in the aetiology of Alzheimer's disease. Nat. Chem. 2009, 1, 326-331.

[32] Fernandez-Lima, F. A., Wei, H., Gao, Y. Q., Russell, D. H., On the structure elucidation using ion mobility spectrometry and molecular dynamics. J. Phys. Chem. A 2009, 113, $8221-8234$.

[33] Politis, A., Park, A. Y., Hyung, S. J., Barsky, D., Ruotolo, B. T., Robinson, C. V., Integrating ion mobility mass spectrometry with molecular modelling to determine the architecture of multiprotein complexes. PLoS One 2010, 5, e12080.

[34] Albrieux, F., Calvo, F., Chirot, F., Vorobyev, A., Tsybin, Y. O., Lepére, V., Antoine, R., Lemoine, J., Dugourd, P., Conformation of polyalanine and polyglycine dications in the gas phase: insight from ion mobility spectrometry and replica-exchange molecular dynamics. J. Phys. Chem. A 2010, 114, 6888-6896.

[35] Jurneczko, E., Barran, P. E., How useful is ion mobility mass spectrometry for structural biology? The relationship between protein crystal structures and their collision cross sections in the gas phase. Analyst 2011, 136, 20-28.

[36] Chirot, F., Calvo, F., Albrieux, F., Lemoine, J. J., Tsybin, Y. O., Dugourd, P., Statistical analysis of ion mobility spectrometry. I. Unbiased and guided replica-exchange molecular dynamics. J. Am. Soc. Mass Spectrom. 2012, 23, 386-396.

[37] Calvo, F., Chirot, F., Albrieux, F., Lemoine, J. J., Tsybin, Y. O., Pernot, P., Dugourd, P., 
Statistical analysis of ion mobility spectrometry. II. Adaptively biased methods and shape correlations. J. Am. Soc. Mass Spectrom. 2012, 23, 1279-1288.

[38] Pacholarz, K. J., Porrini, M., Garlish, R. A., Burnley, R. J., Taylor, R. J., Henry, A. J., Barran, P. E., Dynamics of intact immunoglobulin-G explored by drift-tube ion-mobility mass spectrometry and molecular modeling. Angew. Chemie Int. Ed. 2014, 53, 77657769.

[39] D’Atri, V., Porrini, M., Rosu, F. F., Gabelica, V. V., Linking molecular models with ion 1955 mobility experiments. Illustration with a rigid nucleic acid structure. J. Mass Spectrom. $2015,50,711-726$.

[40] Ghassabi Kondalaji, S., Khakinejad, M., Tafreshian, A., J. Valentine, S., Comprehensive peptide ion structure studies using ion mobility techniques: Part 1. An advanced protocol for molecular dynamics simulations and collision cross-section calculation. J. Am. Soc. Mass Spectrom. 2017, 28, 947-959.

[41] Ruotolo, B. T., Giles, K., Campuzano, I., Sandercock, A. M., Bateman, R. H., Robinson,

[42] Orozco, M., Eisenmesser, A theoretical view of protein dynamics. Chem. Soc. Rev. 1964 2014, 43, 5051-5066.

[43] Mesleh, M. F., Hunter, J. M., Shvartsburg, A. A., Schatz, G. C., Jarrold, M. F., Structural 1966 information from ion mobility measurements: effects of the long-range potential. J. Phys. Chem. A 1997, 101, 968-968.

[44] Shvartsburg, A. a., Schatz, G. C., Jarrold, M. F., Mobilities of carbon cluster ions: Critical importance of the molecular attractive potential. J. Chem. Phys. 1998, 108, 2416-2423.

[45] Wyttenbach, T., Helden, G., Batka, J. J., Carlat, D., Bowers, M. T., Effect of the longrange potential on ion mobility measurements. J. Am. Soc. Mass Spectrom. 1997, 8, 275-282.

[46] Mack, E., Average cross-sectional areas of molecules by gaseous diffusion methods. J. Am. Chem. Soc. 1925, 47, 2468-2482. 
1975 [47] The Martin Jarrold Research Group, http://www.indiana.edu/ nano/software/ (accessed on May 10, 2017) (last time accessed: July 25, 2017).

1977 [48] Shvartsburg, A. A., Jarrold, M. F., An exact hard-spheres scattering model for the mobilities of polyatomic ions. Chem. Phys. Lett. 1996, 261, 86-91.

[49] Marklund, E. G., Degiacomi, M. T., Robinson, C. V., Baldwin, A. J., Benesch, J. L. P., Collision cross sections for structural proteomics. Structure 2015, 23, 791-799.

[50] Campuzano, I., Bush, M. F., Robinson, C. V., Beaumont, C., Richardson, K., Kim, H., 1982

[51] Siu, C. K., Guo, Y., Saminathan, I. S., Hopkinson, A. C., Siu, K. W. M., Optimization of parameters used in algorithms of ion-mobility calculation for conformational analyses. J. Phys. Chem. B 2010, 114, 1204-1212.

[52] Shvartsburg, A. A., Mashkevich, S. V., Baker, E. S., Smith, R. D., Optimization of 1989 algorithms for ion mobility calculations. J. Phys. Chem. A 2007, 111, 2002-2010.

[53] Kim, H., Kim, H. I., Johnson, P. V., Beegle, L. W., Beauchamp, J. L., Goddard, W. A., 1992 Kanik, I., Experimental and theoretical investigation into the correlation between mass and ion mobility for choline and other ammonium cations in N2. Anal. Chem. 2008, 80, 1928-1936.

[55] Bleiholder, C., Wyttenbach, T., Bowers, M. T., A novel projection approximation algorithm for the fast and accurate computation of molecular collision cross sections (I). Method. Int. J. Mass Spectrom. 2011, 308, 1-10.

[56] Bleiholder, C., Contreras, S., Do, T. D., Bowers, M. T., A novel projection approximation algorithm for the fast and accurate computation of molecular collision cross sections (II). Model parameterization and definition of empirical shape factors for proteins. Int. J. 
2004 [57] Bleiholder, C., Contreras, S., Bowers, M. T., A novel projection approximation algorithm 2005 for the fast and accurate computation of molecular collision cross sections (IV). Application to polypeptides. Int. J. Mass Spectrom. 2013, 354-355, 275-280.

2007

[58] Bleiholder, C., A local collision probability approximation for predicting momentum 2008 transfer cross sections. Analyst 2015, 140, 6804-6813.

2009

[59] Larriba-Andaluz, C., Fernandez-Garcia, J., Ewing, M. A., Hogan, C. J., Clemmer, D. E., 2010 Gas molecule scattering \& ion mobility measurements for organic macro-ions in $\mathrm{He}$ versus N2 environments. Phys. Chem. Chem. Phys. 2015, 17, 15019-15029.

2013

[60] Ewing, S. A., Donor, M. T., Wilson, J. W., Prell, J. S., Collidoscope: an improved tool for computing collisional cross-sections with the trajectory method. J. Am. Soc. Mass Spectrom. 2017, 587-596.

[61] Asbury, G. R., Hill, H. H., Evaluation of ultrahigh resolution ion mobility spectrometry as 2016 2017 an analytical separation device in chromatographic terms. J. Microcolumn Sep. 2000, $12,172-178$.

[62] Spangler, G. E., Collins, C. I., Peak shape analysis and plate theory for plasma chromatography. Anal. Chem. 1975, 47, 403-407.

[63] Rokushika, S., Hatano, H., Baim, M. A., Hill, H. H., Resolution measurement for ion 2021 mobility spectrometry. Anal. Chem. 1985, 57, 1902-1907.

2022 [64] Kanu, A. B., Gribb, M. M., Hill, H. H., Predicting optimal resolving power for ambient 2023 pressure ion mobility spectrometry. Anal. Chem. 2008, 80, 6610-6619.

2024 [65] Siems, W. F., Wu, C., Tarver, E. E., Hill, H. H. J., Larsen, P. R., McMinn, D. G., 2025 Measuring the resolving power of ion mobility spectrometers. Anal. Chem. 1994, 66, 4195-4201.

[66] May, J. C., Dodds, J. N., Kurulugama, R. T., Stafford, G. C., Fjeldsted, J. C., McLean, J. A., Broadscale resolving power performance of a high precision uniform field ion mobility-mass spectrometer. Analyst 2015, 140, 6824-6833.

2030 [67] Shvartsburg, A. A. A., Smith, R. R. D., Fundamentals of Traveling Wave lon Mobility 
Spectrometry. Anal. Chem. 2008, 80, 9689-9699.

[68] Zhong, Y., Hyung, S.-J., Ruotolo, B. T., Characterizing the resolution and accuracy of a 2033 second-generation traveling-wave ion mobility separator for biomolecular ions. Analyst $2011,136,3534$.

2035

[69] Hernandez,

D. R., DeBord, J.

D., Ridgeway, M. E., Kaplan, D. A., Park, M. A., Fernandez-Lima, F., lon dynamics in a trapped ion mobility spectrometer. Analyst 2014, 139, 1913-1921.

[71] Silveira, J. A., Michelmann, K., Ridgeway, M. E., Park, M. A., Fundamentals of trapped ion mobility spectrometry part II: fluid dynamics. J. Am. Soc. Mass Spectrom. 2016, 27, $585-595$.

[72] Dwivedi, P., Bendiak, B., Clowers, B. H., Hill, H. H., Rapid resolution of carbohydrate isomers by electrospray ionization ambient pressure ion mobility spectrometry-time-offlight mass spectrometry (ESI-APIMS-TOFMS). J. Am. Soc. Mass Spectrom. 2007, 18,

[73] Deng, L., Ibrahim, Y. M., Hamid, A. M., Garimella, S. V. B., Webb, I. K., Zheng, X., Prost, 2048 S. A., Sandoval, J. A., Norheim, R. V., Anderson, G. A., Tolmachev, A. V., Baker, E. S., Smith, R. D., Ultra-high resolution ion mobility separations utilizing traveling waves in a $13 \mathrm{~m}$ serpentine path length structures for lossless ion manipulations module. Anal. Chem. 2016, 88, 8957-8964.

[74] Silveira, J. A., Danielson, W., Ridgeway, M. E., Park, M. A., Altering the mobility-time continuum: nonlinear scan functions for targeted high resolution trapped ion mobilitymass spectrometry. Int. J. Ion Mobil. Spectrom. 2016, 19, 87-94. $30,556-570$.

2057 [76] Rus, J., Moro, D., Sillero, J. A., Royuela, J., Casado, A., Estevez-Molinero, F., Fernández de la Mora, J., IMS-MS studies based on coupling a differential mobility 
analyzer (DMA) to commercial API-MS systems. Int. J. Mass Spectrom. 2010, 298, 3040.

2061 [77] Shvartsburg, A. A., Tang, K., Smith, R. D., Modeling the resolution and sensitivity of 2062 FAIMS analyses. J. Am. Soc. Mass Spectrom. 2004, 15, 1487-1498.

2063

[78] Santiago, B. G., Harris, R. a, Isenberg, S. L., Glish, G. L., Resolving powers of $>7900$ 2064 using linked scans: how well does resolving power describe the separation capability of differential ion mobility spectrometry. Analyst 2015, 140, 6871-6878.

[79] Tang, F., Xu, C., Wang, X., Resolution enhancement of field asymmetric waveform ion 2068

[80] Ruotolo, B. T., Gillig, K. J., Stone, E. G., Russell, D. H., Peak capacity of ion mobility 2069 mass spectrometry: J. Chromatogr. B 2002, 782, 385-392.

[81] Ruotolo, B. T., McLean, J. A., Gillig, K. J., Russell, D. H., Peak capacity of ion mobility 2071

[85] Dodds, J. N., May, J. C., McLean, J. A., Investigation of the complete suite of the leucine and isoleucine isomers: toward prediction of ion mobility separation capabilities. Anal. Chem. 2017, 89, 952-959.

[86] Mccullagh, M., Goscinny, S., Hanot, V., Roberts, D., Neeson, K., Goshawk, J., Eatough, 2085

[82] Causon, T. J., Hann, S., Theoretical evaluation of peak capacity improvements by use of liquid chromatography combined with drift tube ion mobility-mass spectrometry. $\mathrm{J}$. Chromatogr. A 2015, 1416, 47-56.

[83] Neue, U. D., Peak capacity in unidimensional chromatography. J. Chromatogr. A 2008, $1184,107-130$.

[84] Karasek, F. W., Final Report. University of Waterloo Research Institute; Waterloo, Ontario, Canada: 1980 . Study of technology relating to plasma chromatography sensing tubes.

D., Stead, S., Rao, R., Discovery of pesticide protomers ssing routine ion mobility screening. Waters Appl. Note 2014, 720005028E, 1-7. 
2087 [87] Campbell, J. L., Le Blanc, J. C. Y., Schneider, B. B., Probing electrospray ionization dynamics using differential mobility spectrometry: the curious case of 4-aminobenzoic acid. Anal. Chem. 2012, 84, 7857-7864.

2090

[88] Tian, Z., Kass, S. R., Gas-phase versus liquid-phase structures by electrospray ionization mass spectrometry. Angew. Chemie - Int. Ed. 2009, 48, 1321-1323.

2092

[89] Warnke, S., Seo, J., Boschmans, J., Sobott, F., Scrivens, J. H., Bleiholder, C., Bowers, 2093 M. T., Gewinner, S., Schöllkopf, W., Pagel, K., von Helden, G., Protomers of

[90] McCullagh, M., Stead, S., Williams, J., Keizer, W. de, Bergwerff, A., Identification of 2097 multiple sites of intra-molecular protonation and different fragmentation patterns within the fluoroquinolone class of antibiotics in porcine muscle extracts using travelling wave ion mobility mass spectrometry. Waters Appl. Note No. 720004720en 2013,

2101 [91] Lalli, P. M., Iglesias, B. A., Toma, H. E., Sa, G. F. De, Daroda, R. J., Filho, C. S., Szulejko, J. E., Eberlin, M. N., Protomers : formation, separation and characterization via travelling wave ion mobility mass spectrometry. 2012, 712-719.

[92] Fiehn, O., Metabolomics - the link between genotypes and phenotypes. Plant Mol. Biol. $2002,48,155-171$.

2106 [93] Wolfender, J.-L., Marti, G., Thomas, A., Bertrand, S., Current approaches and challenges for the metabolite profiling of complex natural extracts. J. Chromatogr. A

2109 [94] Cajka, T., Fiehn, O., Toward merging untargeted and targeted methods in mass spectrometry-based metabolomics and lipidomics. Anal. Chem. 2016, 88, 524-545.

[95] Ortmayr, K., Causon, T. J., Hann, S., Koellensperger, G., Increasing selectivity and coverage in LC-MS based metabolome analysis. TrAC Trends Anal. Chem. 2016, 82, 358-366.

2114 [96] Dwivedi, P., Schultz, A. J., Metabolic profiling of human blood by high-resolution ion 
mobility mass spectrometry (IM-MS). Int. J. Mass Spectrom. 2010, 298, 78-90.

2116 [97] Harry, E. L., Weston, D. J., Bristow, A. W. T., Wilson, I. D., Creaser, C. S., An approach to enhancing coverage of the urinary metabonome using liquid chromatography-ion mobility-mass spectrometry. J. Chromatogr. B 2008, 871, 357-361.

[98] Paglia, G., Williams, J. P., Menikarachchi, L., Thompson, J. W., Tyldesley-Worster, R., Halldórsson, S., Rolfsson, O., Moseley, A., Grant, D., Langridge, J., Palsson, B. O.,

[99] Rainville, P. D., Wilson, I. D., Nicholson, J. K., Issacs, G., Mullin, L., Langridge, J. I., Plumb, R. S., Ion mobility spectrometry combined with ultra performance liquid chromatography/mass spectrometry for metabolic phenotyping of urine: Effects of column length, gradient duration and ion mobility spectrometry on metabolite detection. Anal. Chim. Acta 2017, 982, 1-8.

2128 [100] Zhang, X., Kew, K., Reisdorph, R., Sartain, M., Powell, R., Armstrong, M., Quinn, K., Cruickshank-Quinn, C., Walmsley, S., Bokatzian, S., Darland, E., Rain, M., Imatani, K., Reisdorph, N., Performance of a high-pressure liquid chromatography-ion mobilitymass spectrometry system for metabolic profiling. Anal. Chem. 2017, 89, 6384-6391.

[101] Burnum-Johnson, K. E., Baker, E. S., Metz, T. O., Characterizing the lipid and metabolite changes associated with placental function and pregnancy complications using ion mobility spectrometry-mass spectrometry and mass spectrometry imaging. Placenta 2017, DOI: 10.1016/j.placenta.2017.03.016.

[102] Tebani, A., Schmitz-Afonso, I., Rutledge, D. N., Gonzalez, B. J., Bekri, S., Afonso, C., Optimization of a liquid chromatography ion mobility-mass spectrometry method for untargeted metabolomics using experimental design and multivariate data analysis. Anal. Chim. Acta 2016, 913, 55-62.

[103] Arthur, K. L., Turner, M. A., Reynolds, J. C., Creaser, C. S., Increasing peak capacity in non-targeted omics applications by combining full scan field asymmetric waveform ion mobility spectrometry with liquid chromatography-mass spectrometry. Anal. Chem. 
2017, 89, 3452-3459.

2144 [104] Causon, T. J., Došen, M., Reznicek, G., Hann, S., Workflow Development for the analysis of phenolic compounds in wine using liquid chromatography combined with drift-tube ion mobility-mass spectrometry. LCGC North Am. 2016, 34, 854-867.

2147 [105] Stephan, S., Jakob, C., Hippler, J., Schmitz, O. J., A novel four-dimensional analytical approach for analysis of complex samples. Anal. Bioanal. Chem. 2016, 408, 37513759.

2150 [106] Liu, W., Zhang, X., Knochenmuss, R., Siems, W. F., Hill, H. H., Multidimensional separation of natural products using liquid chromatography coupled to hadamard transform ion mobility mass spectrometry. J. Am. Soc. Mass Spectrom. 2016, 27, 810821.

2154 [107] Xiao, X., Miller, L. L., Bernstein, R., Hochrein, J. M., Thermal degradation of $\beta$-carotene studied by ion mobility atmospheric solid analysis probe mass spectrometry: full product pattern and selective ionization enhancement. J. Mass Spectrom. 2016, 51, 309-318.

2157 [108] Covington, B. C., McLean, J. A., Bachmann, B. O., Comparative mass spectrometrybased metabolomics strategies for the investigation of microbial secondary metabolites. Nat. Prod. Rep. 2017, 34, 6-24.

[109] Kylli, P., Hankemeier, T., Kostiainen, R., Feasibility of ultra-performance liquid chromatography-ion mobility-time-of-flight mass spectrometry in analyzing oxysterols.

2163 [110] Chouinard, C. D., Wei, M. S., Beekman, C. R., Kemperman, R. H. J., Yost, R. A., Ion 2164 mobility in clinical analysis: current progress and future perspectives. Clin. Chem. 2016, $62,124-133$.

[111] Cohen, A., Ross, N. W., Smith, P. M., Fawcett, J. P., Analysis of 17ß-estradiol, estriol and estrone in American eel ( Anguilla rostrata) tissue samples using liquid chromatography coupled to electrospray differential ion mobility tandem mass spectrometry. Rapid Commun. Mass Spectrom. 2017, 31, 842-850.

[112] Reading, E., Munoz-Muriedas, J., Roberts, A. D., Dear, G. J., Robinson, C. V., 
Beaumont, C., Elucidation of drug metabolite structural isomers using molecular modeling coupled with ion mobility mass spectrometry. Anal. Chem. 2016, 88, 22732280.

2174 [113] Lee, H. H., Hong, A., Cho, Y., Kim, S., Kim, W. J., Kim, H. I., Structural characterization of anticancer drug paclitaxel and its metabolites using ion mobility mass spectrometry and tandem mass spectrometry. J. Am. Soc. Mass Spectrom. 2016, 27, 329-338.

2177 [114] Beucher, L., Dervilly-Pinel, G., Prévost, S., Monteau, F., Le Bizec, B., Determination of a large set of $\beta$-adrenergic agonists in animal matrices based on ion mobility and mass separations. Anal. Chem. 2015, 87, 9234-9242.

2180 [115] Dwek, R. A., Glycobiology: toward understanding the function of sugars. Chem. Rev. 1996, 96, 683-720.

2182 [116] Varki, A., Biological roles of glycans. Glycobiology 2017, 27, 3-49.

2183 [117] Guile, G. R., Rudd, P. M., Wing, D. R., Prime, S. B., Dwek, R. A., A rapid high-resolution high-performance liquid chromatographic method for separating glycan mixtures and analyzing oligosaccharide profiles. Anal. Biochem. 1996, 240, 210-226.

2186 [118] Picmanova, M., Neilson, E. H., Motawia, M. S., Olsen, C. E., Agerbirk, N., Gray, C. J., Flitsch, S., Meier, S., Silvestro, D., Jorgensen, K., Sanchez-Pérez, R., Moller, B. L., Bjarnholt, N., A recycling pathway for cyanogenic glycosides evidenced by the

[119] Marino, K., Bones, J., Kattla, J. J., Rudd, P. M., A systematic approach to protein glycosylation analysis: a path through the maze. Nat. Chem. Biol. 2010, 6, 713-723.

2193 [120] Hofmann, J., Pagel, K., Glycan analysis by ion mobility-mass spectrometry. Angew. Chemie Int. Ed. 2017, 56, 8342-8349.

2195 [121] Liu, Y., Clemmer, D. E., Characterizing oligosaccharides using injected-ion mobility/mass spectrometry. Anal. Chem. 1997, 69, 2504-2509.

2197 [122] Lee, D.-S., Wu, C., Hill, H. H., Detection of carbohydrates by electrospray ionization-ion mobility spectrometry following microbore high-performance liquid chromatography. $J$. 
Chromatogr. A 1998, 822, 1-9.

2200 [123] Hoffmann, W., Hofmann, J., Pagel, K., Energy-resolved ion mobility-mass spectrometry - A concept to improve the separation of isomeric carbohydrates. J. Am. Soc. Mass Spectrom. 2014, 25, 471-479.

2203

[124] Li, H., Giles, K., Bendiak, B., Kaplan, K., Siems, W. F., Hill, H. H., Resolving structural 2204 isomers of monosaccharide methyl glycosides using drift tube and traveling wave ion mobility mass spectrometry. Anal. Chem. 2012, 84, 3231-3239.

2206

[127] Both, P., Green, A. P., Gray, C. J., Sardzik, R., Voglmeir, J., Fontana, C., Austeri, M.,

[125] Fenn, L. S., McLean, J. A., Structural resolution of carbohydrate positional and structural isomers based on gas-phase ion mobility-mass spectrometry. Phys. Chem. Chem. Phys. 2011, 13, 2196-2205.

[126] Hofmann, J., Hahm, H. S., Seeberger, P. H., Pagel, K., Identification of carbohydrate anomers using ion mobility-mass spectrometry. Nature 2015, 526, 241-244. Rejzek, M., Richardson, D., Field, R. A., Widmalm, G., Flitsch, S. L., Eyers, C. E., Discrimination of epimeric glycans and glycopeptides using IM-MS and its potential for carbohydrate sequencing. Nat. Chem. 2013, 6, 65-74.

[128] Li, H., Bendiak, B., Siems, W. F., Gang, D. R., Hill, H. H., Determining the isomeric heterogeneity of neutral oligosaccharide-alditols of bovine submaxillary mucin using negative ion traveling wave ion mobility mass spectrometry. Anal. Chem. 2015, 87, 2228-2235.

[129] Harvey, D. J., Scarff, C. A., Edgeworth, M., Crispin, M., Scanlan, C. N., Sobott, F., Allman, S., Baruah, K., Pritchard, L., Scrivens, J. H., Travelling wave ion mobility and negative ion fragmentation for the structural determination of $\mathrm{N}$-linked glycans. Electrophoresis 2013, 34, 2368-2378.

[130] Harvey, D. J., Scarff, C. A., Edgeworth, M., Pagel, K., Thalassinos, K., Struwe, W. B., Crispin, M., Scrivens, J. H., Travelling-wave ion mobility mass spectrometry and negative ion fragmentation of hybrid and complex N -glycans. J. Mass Spectrom. 2016, $51,1064-1079$. 
2227 [131] Sarbu, M., Zhu, F., Peter-Katalinić, J., Clemmer, D. E., Zamfir, A. D., Application of ion mobility tandem mass spectrometry to compositional and structural analysis of glycopeptides extracted from the urine of a patient diagnosed with Schindler disease. Rapid Commun. Mass Spectrom. 2015, 29, 1929-1937.

2231

[132] Pu, Y., Ridgeway, M. E., Glaskin, R. S., Park, M. A., Costello, C. E., Lin, C., Separation 2232 and identification of isomeric glycans by selected accumulation-trapped ion mobility spectrometry-electron activated dissociation tandem mass spectrometry. Anal. Chem. 2016, 88, 3440-3443.

2235

[133] Lee, S., Valentine, S. J., Reilly, J. P., Clemmer, D. E., Analyzing a mixture of 2236 disaccharides by IMS-VUVPD-MS. Int. J. Mass Spectrom. 2012, 309, 161-167.

[134] Ropartz, D., Giuliani, A., Fanuel, M., Hervé, C., Czjzek, M., Rogniaux, H., Online 2238 coupling of high-resolution chromatography with extreme UV photon activation tandem mass spectrometry: Application to the structural investigation of complex glycans by dissociative photoionization. Anal. Chim. Acta 2016, 933, 1-9.

[135] Li, H., Bendiak, B., Siems, W. F., Gang, D. R., Hill, H. H., Carbohydrate structure characterization by tandem ion mobility mass spectrometry. Anal. Chem. 2013, 85, $2760-2769$.

[136] Gaye, M. M., Kurulugama, R., Clemmer, D. E., Investigating carbohydrate isomers by IMS-CID-IMS-MS: precursor and fragment ion cross-sections. Analyst 2015, 140, 69226932.

2247 [137] Paglia, G., Kliman, M., Claude, E., Geromanos, S., Astarita, G., Applications of ion2248 mobility mass spectrometry for lipid analysis. Anal. Bioanal. Chem. 2015, 407, 49955007.

[138] Paglia, G., Shrestha, B., Astarita, G., Ion-mobility mass spectrometry for lipidomics 2251 applications. 2017, pp. 61-79.

2252 [139] Hines, K. M., May, J. C., McLean, J. A., Xu, L., Evaluation of collision cross section 2253 calibrants for structural analysis of lipids by traveling wave ion mobility-mass spectrometry. Anal. Chem. 2016, 88, 7329-7336. 
2255 [140] Hankin, J. A., Barkley, R. M., Zemski-Berry, K., Deng, Y., Murphy, R. C., Mass spectrometric collisional activation and product ion mobility of human serum neutral lipid extracts. Anal. Chem. 2016, 88, 6274-6282.

[141] Berry, K. A. Z., Barkley, R. M., Berry, J. J., Hankin, J. A., Hoyes, E., Brown, J. M., 2259 Murphy, R. C., Tandem mass spectrometry in combination with product ion mobility for the identification of phospholipids. Anal. Chem. 2017, 89, 916-921.

2261

[142] Kyle, J. E., Zhang, X., Weitz, K. K., Monroe, M. E., Ibrahim, Y. M., Moore, R. J., Cha, 2262

[143] Kyle, J. E., Casey, C. P., Stratton, K. G., Zink, E. M., Kim, Y.-M., Zheng, X., Monroe, M. E., Weitz, K. K., Bloodsworth, K. J., Orton, D. J., Ibrahim, Y. M., Moore, R. J., Lee, C. G., Pedersen, C., Orwoll, E., Smith, R. D., Burnum-Johnson, K. E., Baker, E. S., Comparing identified and statistically significant lipids and polar metabolites in 15-year old serum and dried blood spot samples for longitudinal studies. Rapid Commun. Mass Spectrom. 2017, 31, 447-456.

[144] Leng, J., Guan, Q., Sun, T., Wang, H., Cui, J., Liu, Q., Zhang, Z., Zhang, M., Guo, Y., Direct infusion electrospray ionization-ion mobility-mass spectrometry for comparative profiling of fatty acids based on stable isotope labeling. Anal. Chim. Acta 2015, 887, $148-154$.

[145] GroessI, M., Graf, S., Knochenmuss, R., High resolution ion mobility-mass spectrometry for separation and identification of isomeric lipids. Analyst 2015, 140, 6904-6911.

[146] Zhou, Z. Z., Zhu, Z.-J., 13th Annual Conference of the Metabolomics Society, Brisbane, Australia. 25-29 June 2017.

2280 [147] Steiner, R., Saied, E. M., Othman, A., Arenz, C., Maccarone, A. T., Poad, B. L. J., 2281 Blanksby, S. J., von Eckardstein, A., Hornemann, T., Elucidating the chemical structure of native 1-deoxysphingosine. J. Lipid Res. 2016, 57, 1194-1203. 
2283 [148] Bowman, A. P., Abzalimov, R. R., Shvartsburg, A. A., Broad separation of isomeric lipids by high-resolution differential ion mobility spectrometry with tandem mass spectrometry. J. Am. Soc. Mass Spectrom. 2017, 28, 1552-1561.

[149] Márquez-Sillero, I., Aguilera-Herrador, E., Cárdenas, S., Valcárcel, M., Ion-mobility spectrometry for environmental analysis. TrAC Trends Anal. Chem. 2011, 30, 677-690.

[150] Lipok, C., Hippler, J., Schmitz, O. J., A four dimensional separation method based on continuous heart-cutting gas chromatography with ion mobility and high resolution mass spectrometry. J. Chromatogr. A 2017, DOI: 10.1016/j.chroma.2017.07.013.

229

[151] Stephan, S., Hippler, J., Köhler, T., Deeb, A. A., Schmidt, T. C., Schmitz, O. J., 2292 Contaminant screening of wastewater with HPLC-IM-qTOF-MS and LC+LC-IM-qTOFMS using a CCS database. Anal. Bioanal. Chem. 2016, 408, 6545-6555.

[152] Grimm, F. A., Russell, W. K., Luo, Y.-S., Iwata, Y., Chiu, W. A., Roy, T., Boogaard, P. J., Ketelslegers, H. B., Rusyn, I., Grouping of petroleum substances as example UVCBs by ion mobility-mass spectrometry to enable chemical composition-based read-across. Environ. Sci. Technol. 2017, 51, 7197-7207.

[153] Metz, T. O., Baker, E. S., Schymanski, E. L., Renslow, R. S., Thomas, D. G., Causon, 2299 T. J., Webb, I. K., Hann, S., Smith, R. D., Teeguarden, J. G., Integrating ion mobility spectrometry into mass spectrometry-based exposome measurements: what can it add and how far can it go? Bioanalysis 2017, 9, 81-98.

[154] Zhou, Z., Shen, X., Tu, J., Zhu, Z.-J., Large-scale prediction of collision cross-section values for metabolites in ion mobility-mass spectrometry. Anal. Chem. 2016, 88, 1108411091.

[155] Zhou, Z., Xiong, X., Zhu, Z.-J., MetCCS predictor: a web server for predicting collision cross-section values of metabolites in ion mobility-mass spectrometry based metabolomics. Bioinformatics 2017, 33, 2235-2237.

[156] Bijlsma, L., Bade, R., Celma, A., Mullin, L., Cleland, G., Stead, S., Hernandez, F., Sancho, J. V., Prediction of collision cross-section values for small molecules: application to pesticide residue analysis. Anal. Chem. 2017, 89, 6583-6589. 
2311 [157] Eiceman, G. A., Shoff, D. B., Harden, C. S., Snyder, A. P., Martinez, P. M., Fleischer, M. E., Watkins, M. L., Ion mobility spectrometry of halothane, enflurane, and isoflurane anesthetics in air and respired gases. Anal. Chem. 1989, 61, 1093-9.

2314 [158] Bennett, R. V., Gamage, C. M., Galhena, A. S., Fernández, F. M., Contrast-enhanced differential mobility-desorption electrospray ionization-mass spectrometry imaging of biological tissues. Anal. Chem. 2014, 86, 3756-3763.

[159] Trim, P. J., Henson, C. M., Avery, J. L., McEwen, A., Snel, M. F., Claude, E., Marshall, 2318

[160] McLean, J. A., Ridenour, W. B., Caprioli, R. M., Profiling and imaging of tissues by imaging ion mobility-mass spectrometry. J. Mass Spectrom. 2007, 42, 1099-1105.

[161] Glover, M. S., Dilger, J. M., Acton, M. D., Arnold, R. J., Radivojac, P., Clemmer, D. E., Examining the influence of phosphorylation on peptide ion structure by ion mobility spectrometry-mass spectrometry. J. Am. Soc. Mass Spectrom. 2016, 27, 786-794.

[162] Ruotolo, B. T., Verbeck, Thomson, L. M., Woods, A. S., Gillig, K. J., Russell, D. H., Distinguishing between phosphorylated and nonphosphorylated peptides with ion mobility-mass spectrometry. J. Proteome Res. 2002, 1, 303-306.

[163] Ruotolo, B. T., Gillig, K. J., Woods, A. S., Egan, T. F., Ugarov, M. V., Schultz, J. A., 2330 Russell, D. H., Analysis of phosphorylated peptides by ion mobility-mass spectrometry. Anal. Chem. 2004, 76, 6727-6733.

[164] Shvartsburg, A. A., Singer, D., Smith, R. D., Hoffmann, R., lon mobility separation of isomeric phosphopeptides from a protein with variant modification of adjacent residues. Anal. Chem. 2011, 83, 5078-5085.

[165] Baird, M. A., Shvartsburg, A. A., Localization of post-translational modifications in peptide mixtures via high-resolution differential ion mobility separations followed by electron transfer dissociation. J. Am. Soc. Mass Spectrom. 2016, 27, 2064-2070.

[166] Shvartsburg, A. A., Zheng, Y., Smith, R. D., Kelleher, N. L., Separation of variant 
methylated histone tails by differential ion mobility. Anal. Chem. 2012, 84, 6317-6320.

2340 [167] Shvartsburg, A. A., Zheng, Y., Smith, R. D., Kelleher, N. L., Ion mobility separation of variant histone tails extending to the "middle-down" Range. Anal. Chem. 2012, 84, $4271-4276$.

[168] Aebersold, R., Mann, M., Mass spectrometry-based proteomics. Nature 2003, 422, 198-207.

[169] Cravatt, B. F., Simon, G. M., Yates III, J. R., The biological impact of mass-spectrometrybased proteomics. Nature 2007, 450, 991-1000.

2347 [170] Altelaar, A. F. M., Munoz, J., Heck, A. J. R., Next-generation proteomics: towards an integrative view of proteome dynamics. Nat. Rev. Genet. 2012, 14, 35-48.

[171] Andrews, G. L., Simons, B. L., Young, J. B., Hawkridge, A. M., Muddiman, D. C., Performance characteristics of a new hybrid quadrupole time-of-flight tandem mass spectrometer (TripleTOF 5600). Anal Chem 2011, 83, 5442-5446.

[172] Beck, S., Michalski, A., Raether, O., Lubeck, M., Kaspar, S., Goedecke, N., Baessmann, C., Hornburg, D., Meier, F., Paron, I., Kulak, N. A., Cox, J., Mann, M., The Impact II, a very high-resolution quadrupole time-of-flight instrument (QTOF) for deep shotgun proteomics. Mol. Cell. Proteomics 2015, 14, 2014-2029.

[173] Hoaglund-Hyzer, C. S., Clemmer, D. E., Ion trap/ion mobility/quadrupole/time-of-flight mass spectrometry for peptide mixture analysis. Anal. Chem. 2001, 73, 177-184.

[174] Shliaha, P. V., Bond, N. J., Gatto, L., Lilley, K. S., Effects of traveling wave ion mobility 2359 separation on data independent acquisition in proteomics studies. J. Proteome Res. 2013, 12, 2323-2339.

2361 [175] Helm, D., Vissers, J. P. C., Hughes, C. J., Hahne, H., Ruprecht, B., Pachl, F., Grzyb, 2362 A., Richardson, K., Wildgoose, J., Maier, S. K., Marx, H., Wilhelm, M., Becher, I., Lemeer, S., Bantscheff, M., Langridge, J. I., Kuster, B., Ion mobility tandem mass spectrometry enhances performance of bottom-up proteomics. Mol. Cell. Proteomics 2014, 13, 3709-3715.

2366 [176] Chapman, J. D., Goodlett, D. R., Masselon, C. D., Multiplexed and data-independent 
tandem mass spectrometry for global proteome profiling. Mass Spectrom. Rev. 2014, 33, 452-470.

2369

[177] Silva, J. C., Denny, R., Dorschel, C. A., Gorenstein, M., Kass, I. J., Li, G. Z., McKenna, T., Nold, M. J., Richardson, K., Young, P., Geromanos, S., Quantitative proteomic analysis by accurate mass retention time pairs. Anal Chem 2005, 77, 2187-2200.

[178] Geromanos, S. J., Hughes, C., Ciavarini, S., Vissers, J. P. C., Langridge, J. I., Using ion purity scores for enhancing quantitative accuracy and precision in complex proteomics samples. Anal. Bioanal. Chem. 2012, 404, 1127-1139.

2375

[179] Daly, C. E., Ng, L. L., Hakimi, A., Willingale, R., Jones, D. J. L., Charlotte E. Daly, Leong 2376 L. Ng, Hakimi, A., Willingale, R., and Jones, D. Qualitative and quantitative characterization of plasma proteins when incorporating traveling wave ion mobility into

[180] Distler, U., Kuharev, J., Navarro, P., Tenzer, S., Label-free quantification in ion mobility 2382 enhanced data-independent acquisition proteomics. Nat. Protoc. 2016, 11, 795-812.

[181] Distler, U., Kuharev, J., Navarro, P., Levin, Y., Schild, H., Tenzer, S., Drift time-specific 2384 collision energies enable deep-coverage data-independent acquisition proteomics. Nat.

2386 [182] Distler, U., Kuharev, J., Tenzer, S., Biomedical applications of ion mobility-enhanced 2387 data-independent acquisition-based label-free quantitative proteomics. Expert Rev.

[183] Souza, G. H. M. F., Guest, P. C., Martins-de-Souza, D., Methods in Molecular Biology. 2390 2017, pp. 57-73.

2391 [184] Haynes, S. E., Polasky, D. A., Dixit, S. M., Majmudar, J. D., Neeson, K., Ruotolo, B. T., Martin, B. R., Variable-velocity traveling-wave ion mobility separation enhancing peak capacity for data-independent acquisition proteomics. Anal. Chem. 2017, acs.analchem.7b00112. 
2395 [185] Meier, F., Beck, S., Grassl, N., Lubeck, M., Park, M. A., Raether, O., Mann, M., Parallel accumulation-serial fragmentation (PASEF): Multiplying sequencing speed and sensitivity by synchronized scans in a trapped ion mobility device. J. Proteome Res. $2015,14,5378-5387$.

[186] Shelimov, K. B., Clemmer, D. E., Hudgins, R. R., Jarrold, M. F., Protein structure in vacuo: gas-phase confirmations of BPTI and cytochrome c. J. Am. Chem. Soc. 1997, $119,2240-2248$.

[187] Wyttenbach, T., Bowers, M. T., Structural stability from solution to the gas phase: Native solution structure of ubiquitin survives analysis in a solvent-free ion mobility-mass spectrometry environment. J. Phys. Chem. B 2011, 115, 12266-12275.

[188] Gonzalez Florez, A. I., Mucha, E., Ahn, D.-S., Gewinner, S., Schollkopf, W., Pagel, K., von Helden, G., Charge-induced unzipping of isolated proteins to a defined secondary structure. Angew. Chemie Int. Ed. 2016, 55, 3295-3299.

[189] Segev, E., Wyttenbach, T., Bowers, M. T., Gerber, R. B., Conformational evolution of ubiquitin ions in electrospray mass spectrometry: molecular dynamics simulations at gradually increasing temperatures. Phys. Chem. Chem. Phys. 2008, 10, 3077.

[190] Allison, T. M., Landreh, M., Benesch, J. L. P., Robinson, C. V., Low charge and reduced mobility of membrane protein complexes has implications for calibration of collision cross section measurements. Anal. Chem. 2016, 88, 5879-5884.

[191] Hall, Z., Politis, A., Bush, M. F., Smith, L. J., Robinson, C. V., Charge-state dependent compaction and dissociation of protein complexes: Insights from ion mobility and molecular dynamics. J. Am. Chem. Soc. 2012, 134, 3429-3438.

[192] Devine, P. W. A., Fisher, H. C., Calabrese, A. N., Whelan, F., Higazi, D. R., Potts, J. R., Lowe, D. C., Radford, S. E., Ashcroft, A. E., Investigating the Structural Compaction of Biomolecules Upon Transition to the Gas-Phase Using ESI-TWIMS-MS. J. Am. Soc. Mass Spectrom. 2017, 1-8.

[193] Seo, J., Hoffmann, W., Warnke, S., Bowers, M. T., Pagel, K., von Helden, G., Retention of native protein structures in the absence of solvent: a coupled ion mobility and 
spectroscopic study. Angew. Chemie Int. Ed. 2016, 55, 14173-14176.

2424 [194] Warnke, S., Hoffmann, W., Seo, J., De Genst, E., von Helden, G., Pagel, K., From compact to string - the role of secondary and tertiary structure in charge-induced unzipping of gas-phase proteins. J. Am. Soc. Mass Spectrom. 2017, 28, 638-646.

[195] Beveridge, R., Chappuis, Q., Macphee, C., Barran, P., Mass spectrometry methods for intrinsically disordered proteins. Analyst 2013, 138, 32-42.

[196] Jurneczko, E., Cruickshank, F., Porrini, M., Nikolova, P., Campuzano, I. D. G., Morris, M., Barran, P. E., Intrinsic disorder in proteins: a challenge for (un)structural biology met by ion mobility-mass spectrometry. Biochem. Soc. Trans. 2012, 40, 1021-1026.

[197] Bernstein, S. L., Liu, D., Wyttenbach, T., Bowers, M. T., Lee, J. C., Gray, H. B., Winkler, J. R., a-Synuclein: stable compact and extended monomeric structures and $\mathrm{pH}$ dependence of dimer formation. J. Am. Soc. Mass Spectrom. 2004, 15, 1435-1443.

[198] Faull, P. A., Florance, H. V., Schmidt, C. Q., Tomczyk, N., Barlow, P. N., Hupp, T. R.,

[199] Maurizio, E., Cravello, L., Brady, L., Spolaore, B., Arnoldo, L., Giancotti, V., Manfioletti, Nikolova, P. V., Barran, P. E., Utilising ion mobility-mass spectrometry to interrogate

[200] Dickinson, E. R., Jurneczko, E., Nicholson, J., Hupp, T. R., Zawacka-Pankau, J., Selivanova, G., Barran, P. E., The use of ion mobility mass spectrometry to probe modulation of the structure of p53 and of MDM2 by small molecule inhibitors. Front. Mol. Biosci. 2015, 2, 1-9.

2448 [201] Knapman, T. W., Valette, N. M., Warriner, S. L., Ashcroft, a E., Ion mobility spectrometry-mass spectrometry of intrinsically unfolded proteins: trying to put order into disorder. Curr. Anal. Chem. 2013, 9, 181-191. 
2451 [202] Hoffmann, W., von Helden, G., Pagel, K., Ion mobility-mass spectrometry and orthogonal gas-phase techniques to study amyloid formation and inhibition. Curr. Opin. Struct. Biol. 2017, 46, 7-15.

[203] Leney, A. C., Pashley, C. L., Scarff, C. A., Radford, S. E., Ashcroft, A. E., Insights into the role of the $\beta-2$ microglobulin D-strand in amyloid propensity revealed by mass spectrometry. Mol. BioSyst. 2014, 10, 412-420.

2457 [204] Young, L. M., Mahood, R. A., Saunders, J. C., Tu, L.-H., Raleigh, D. P., Radford, S. E., Ashcroft, A. E., Insights into the consequences of co-polymerisation in the early stages of IAPP and $\alpha \beta$ peptide assembly from mass spectrometry. Analyst $2015,140,6990$ 6999.

2461 [205] Dupuis, N. F., Wu, C., Shea, J., Bowers, M. T., $\beta$-hairpins: a possible direct amyloidogenic precursor. 2009, 18283-18292.

2463 [206] Dupuis, N. F., Wu, C., Shea, J. E., Bowers, M. T., The amyloid formation mechanism in human IAPP: dimers have beta-strand monomer-monomer interfaces. J Am Chem Soc $2011,133,7240-7243$.

[207] Cole, H. L., Kalapothakis, J. M. D., Bennett, G., Barran, P. E., MacPhee, C. E., Characterizing early aggregates formed by an amyloidogenic peptide by mass spectrometry. Angew. Chemie - Int. Ed. 2010, 49, 9448-9451.

[208] Illes-Toth, E., Ramos, M. R., Cappai, R., Dalton, C., Smith, D. P., Distinct higher-order a-synuclein oligomers induce intracellular aggregation. Biochem. J. 2015, 468, 485493.

[209] Daly, S., Kulesza, A., Poussigue, F., Simon, A.-L., Choi, C. M., Knight, G., Chirot, F., MacAleese, L., Antoine, R., Dugourd, P., Correction: Conformational changes in amyloid- $\beta$ alloforms studied using action-FRET, IMS and molecular dynamics simulations. Chem. Sci. 2016, 7, 1609-1610.

[210] Seo, J., Hoffmann, W., Warnke, S., Huang, X., Gewinner, S., Schöllkopf, W., Bowers, M. T., von Helden, G., Pagel, K., An infrared spectroscopy approach to follow $\beta$-sheet formation in peptide amyloid assemblies. Nat. Chem. 2016, 9, 39-44. 
2479 [211] Young, L. M., Saunders, J. C., Mahood, R. A., Revill, C. H., Foster, R. J., Tu, L.-H., Raleigh, D. P., Radford, S. E., Ashcroft, A. E., Screening and classifying small-molecule inhibitors of amyloid formation using ion mobility spectrometry-mass spectrometry. Nat. Chem. 2014, 7, 73-81.

[212] Young, L. M., Saunders, J. C., Mahood, R. A., Revill, C. H., Foster, R. J., Ashcroft, A. E., Radford, S. E., ESI-IMS-MS: a method for rapid analysis of protein aggregation and its inhibition by small molecules. Methods 2016, 95, 62-69.

[213] Eschweiler, J. D., Kerr, R., Rabuck-Gibbons, J., Ruotolo, B. T., Sizing up protein-ligand complexes: the rise of structural mass spectrometry approaches in the pharmaceutical sciences. Annu. Rev. Anal. Chem. 2017, 10, 25-44.

[214] Göth, M., Pagel, K., lon mobility-mass spectrometry as a tool to investigate proteinligand interactions. Anal. Bioanal. Chem. 2017, 409, 4305-4310.

[215] Stojko, J., Fieulaine, S., Petiot-Bocard, S., Van Dorsselaer, A., Meinnel, T., Giglione, C., Cianférani, S., Ion mobility coupled to native mass spectrometry as a relevant tool to investigate extremely small ligand-induced conformational changes. Analyst 2015, 140,

[216] Harvey, S. R., Porrini, M., Konijnenberg, A., Clarke, D. J., Tyler, R. C., Langridge-Smith, P. R. R., Macphee, C. E., Volkman, B. F., Barran, P. E., Dissecting the dynamic conformations of the metamorphic protein lymphotactin. J. Phys. Chem. B 2014, 118,

[218] Harvey, S. R., Porrini, M., Stachl, C., MacMillan, D., Zinzalla, G., Barran, P. E., Small$12348-12359$.

[217] Rabuck, J. N., Hyung, S. J., Ko, K. S., Fox, C. C., Soellner, M. B., Ruotolo, B. T., Activation state-selective kinase inhibitor assay based on ion mobility-mass spectrometry. Anal. Chem. 2013, 85, 6995-7002. molecule inhibition of c-MYC:MAX leucine zipper formation is revealed by ion mobility mass spectrometry. J. Am. Chem. Soc. 2012, 134, 19384-19392.

[219] Hopper, J. T. S., Oldham, N. J., Collision induced unfolding of protein ions in the gas phase studied by ion mobility-mass spectrometry: the effect of ligand binding on 
conformational stability. J. Am. Soc. Mass Spectrom. 2009, 20, 1851-1858.

2508 [220] Niu, S., Rabuck, J. N., Ruotolo, B. T., lon mobility-mass spectrometry of intact protein2509 ligand complexes for pharmaceutical drug discovery and development. Curr. Opin. Chem. Biol. 2013, 17, 809-817.

2512

[221] Laganowsky, A., Reading, E., Allison, T. M., Ulmschneider, M. B., Degiacomi, M. T., Baldwin, A. J., Robinson, C. V., Membrane proteins bind lipids selectively to modulate

2514

2515

2516 their structure and function. Nature 2014, 510, 172-175.

[222] Niu, S., Ruotolo, B. T., Collisional unfolding of multiprotein complexes reveals cooperative stabilization upon ligand binding. Protein Sci. 2015, 24, 1272-1281.

[223] Barrera, N. P., Di Bartolo, N., Booth, P. J., Robinson, C. V., Micelles protect membrane complexes from solution to vacuum. Science (80-. ). 2008, 321, 243-246.

[224] Wang, S. C., Politis, A., Di Bartolo, N., Bavro, V. N., Tucker, S. J., Booth, P. J., Barrera, N. P., Robinson, C. V., Ion mobility mass spectrometry of two tetrameric membrane protein complexes reveals compact structures and differences in stability and packing. J. Am. Chem. Soc. 2010, 132, 15468-15470.

[225] Liu, Y., Cong, X., Liu, W., Laganowsky, A., Characterization of membrane protein-lipid interactions by mass spectrometry ion mobility mass spectrometry. J. Am. Soc. Mass Spectrom. 2017, 28, 579-586.

[226] Lee, A. G., Biological membranes: the importance of molecular detail. Trends Biochem. Sci. 2011, 36, 493-500.

[227] Long, S. B., Tao, X., Campbell, E. B., MacKinnon, R., Atomic structure of a voltagedependent K+ channel in a lipid membrane-like environment. Nature 2007, 450, 376382.

[228] Popot, J.-L., Amphipols, nanodiscs, and fluorinated surfactants: three non conventional approaches to studying membrane proteins in aqueous solutions. Annu. Rev. Biochem. 2010, 79, 737-775.

[229] Watkinson, T. G., Calabrese, A. N., Giusti, F., Zoonens, M., Radford, S. E., Ashcroft, A. E., Systematic analysis of the use of amphipathic polymers for studies of outer 
membrane proteins using mass spectrometry. Int. J. Mass Spectrom. 2015, 391, 5461.

2537 [230] Singer, S. J., Nicolson, G. L., The fluid mosaic model of the structure of cell membranes. Science. 1972, 175, 720-731.

[231] HUNTE, C., RICHERS, S., Lipids and membrane protein structures. Curr. Opin. Struct. Biol. 2008, 18, 406-411.

2541 [232] Bush, M. F., Hall, Z., Giles, K., Hoyes, J., Robinson, C. V., Ruotolo, B. T., Collision Cross Sections of Proteins and Their Complexes: A Calibration framework and database for gas-phase structural biology. Anal. Chem. 2010, 82, 9557-9565.

[233] Smith, D., Knapman, T., Campuzano, I., Malham, R., Berryman, J., Radford, S., Ashcroft, A., Deciphering drift time measurements from travelling wave ion mobility spectrometry-mass spectrometry studies. Eur. J. Mass Spectrom. 2009, 15, 113.

[234] van Duijn, E., Barbu, I. M., Barendregt, A., Jore, M. M., Wiedenheft, B., Lundgren, M., 2548 Westra, E. R., Brouns, S. J. J., Doudna, J. A., van der Oost, J., Heck, A. J. R., Native

[235] Politis, A., Park, A. Y., Hall, Z., Ruotolo, B. T., Robinson, C. V., Integrative modelling 2554 tandem and ion mobility mass spectrometry highlight structural and modular similarities in clustered-regularly-interspaced shot-palindromic-repeats (CRISPR) - associated protein complexes from Escherichia coli and Pseudomonas aeruginosa. Mol. Cell. Proteomics 2012, 11, 1430-1441.

[236] Saliou, J.-M., Manival, X., Tillault, A.-S., Atmanene, C., Bobo, C., Branlant, C., Van 2558 coupled with ion mobility mass spectrometry reveals structural features of the clamp loader in complex with single-stranded DNA binding protein. J. Mol. Biol. 2013, 425, $4790-4801$.

[237] Uetrecht, C., Versluis, C., Watts, N., Wingfield, P., Steven, A., Heck, A. R., Stability and shape of hepatitis B virus capsids in vacuo. Angew. Chemie Int. Ed. 2008, 47, 6247- 
6251.

2564 [238] Shepherd, D., Holmes, K., Rowlands, D., Stonehouse, N., Ashcroft, A., Using ion 2565 mobility spectrometry-mass spectrometry to decipher the conformational and assembly characteristics of the hepatitis B capsid protein. Biophys. J. 2013, 105, 1258-1267.

[239] Snijder, J., van de Waterbeemd, M., Damoc, E., Denisov, E., Grinfeld, D., Bennett, A., Agbandje-McKenna, M., Makarov, A., Heck, A. J. R., Defining the stoichiometry and cargo load of viral and bacterial nanoparticles by orbitrap mass spectrometry. J. Am. Chem. Soc. 2014, 136, 7295-7299.

257

[240] Benigni, P., Marin, R., Molano-Arevalo, J. C., Garabedian, A., Wolff, J. J., Ridgeway, M. E., Park, M. A., Fernandez-Lima, F., Towards the analysis of high molecular weight proteins and protein complexes using TIMS-MS. Int. J. Ion Mobil. Spectrom. 2016, 19, 95-104.

[241] Quintyn, R. S., Harvey, S. R., Wysocki, V. H., Illustration of SID-IM-SID (surface-induced dissociation-ion mobility-SID) mass spectrometry: homo and hetero model protein complexes. Analyst 2015, 140, 7012-7019.

[242] Quintyn, R. S., Zhou, M., Yan, J., Wysocki, V. H., Surface-induced dissociation mass spectra as a tool for distinguishing different structural forms of gas-phase multimeric protein complexes. Anal. Chem. 2015, 87, 11879-11886.

[243] Samulak, B. M., Niu, S., Andrews, P. C., Ruotolo, B. T., Ion mobility-mass spectrometry analysis of cross-linked intact multiprotein complexes: enhanced gas-phase stabilities and altered dissociation pathways. Anal. Chem. 2016, 88, 5290-5298.

[244] Marcoux, J., Cianférani, S., Towards integrative structural mass spectrometry: benefits from hybrid approaches. Methods 2015, 89, 4-12.

[245] Politis, A., Borysik, A. J., Assembling the pieces of macromolecular complexes: hybrid structural biology approaches. Proteomics 2015, 15, 2792-2803.

[246] Politis, A., Stengel, F., Hall, Z., Hernandez, H., Leitner, A., Walzthoeni, T., Robinson, C. V, Aebersold, R., A mass spectrometry-based hybrid method for structural modeling of protein complexes. Nat. Methods 2014, 11, 403-406. 
[247] Marcoux, J., Politis, A., Rinehart, D., Marshall, D. P., Wallace, M. I., Tamm, L. K., 2592 Robinson, C. V., Mass spectrometry defines the C-terminal dimerization domain and enables modeling of the structure of full-length OmpA. Structure 2014, 22, 781-790.

[248] Tsutsui, Y., Sarkar, A., Wintrode, P. L., Probing serpin conformational change using mass spectrometry and related methods. Elsevier Inc. 2011.

[249] Snijder, J., Burnley, R. J., Wiegard, A., Melquiond, A. S. J., Bonvin, A. M. J. J., Axmann, I. M., Heck, A. J. R., Insight into cyanobacterial circadian timing from structural details of the KaiB-KaiC interaction. Proc. Natl. Acad. Sci. 2014, 111, 1379-1384.

[250] Beck, A., Terral, G., Debaene, F., Wagner-Rousset, E., Marcoux, J., Janin-Bussat, M.C., Colas, O., Dorsselaer, A. Van, Cianférani, S., Cutting-edge mass spectrometry methods for the multi-level structural characterization of antibody-drug conjugates. Expert Rev. Proteomics 2016, 13, 157-183.

[251] Beck, A., Sanglier-Cianférani, S., Van Dorsselaer, A., Biosimilar, biobetter, and next generation antibody characterization by mass spectrometry. Anal. Chem. 2012, 84, $4637-4646$.

[252] Hyung, S.-J., Robinson, C. V., Ruotolo, B. T., Gas-phase unfolding and disassembly reveals stability differences in ligand-bound multiprotein complexes. Chem. Biol. 2009, $16,382-390$.

[253] Bagal, D., Valliere-Douglass, J. F., Balland, A., Schnier, P. D., Resolving disulfide structural isoforms of IgG2 monoclonal antibodies by ion mobility mass spectrometry. Anal. Chem. 2010, 82, 6751-6755.

[254] Jones, L. M., Zhang, H., Cui, W., Kumar, S., Sperry, J. B., Carroll, J. A., Gross, M. L., Complementary MS methods assist conformational characterization of antibodies with

2615 [255] Pritchard, C., Groves, K. J., Biesenbruch, S., O'Connor, G., Ashcroft, A. E., Arsene, C., Schulze, D., Quaglia, M., Quantification of human growth hormone in serum with a labeled protein as an internal standard: essential considerations. Anal. Chem. 2014, 86, 6525-6532. 
2619 [256] Debaene, F., Wagner-Rousset, E., Colas, O., Ayoub, D., Corvaia, N., Van Dorsselaer, 2620 A., Beck, A., Cianférani, S., Time resolved native ion-mobility mass spectrometry to monitor dynamics of IgG4 Fab arm exchange and "bispecific" monoclonal antibody formation. Anal. Chem. 2013, 85, 9785-9792.

2624

2625

2626

2627

2628

[257] Debaene, F., Bœuf, A., Wagner-Rousset, E., Colas, O., Ayoub, D., Corvaïa, N., Van Dorsselaer, A., Beck, A., Cianférani, S., Innovative native MS methodologies for antibody drug conjugate characterization: high resolution native MS and IM-MS for average DAR and DAR distribution assessment. Anal. Chem. 2014, 86, 10674-10683.

[258] Marcoux, J., Champion, T., Colas, O., Wagner-Rousset, E., Corvaïa, N., Van Dorsselaer, A., Beck, A., Cianférani, S., Native mass spectrometry and ion mobility 2629 characterization of trastuzumab emtansine, a lysine-linked antibody drug conjugate. Protein Sci. 2015, 24, 1210-1223.

2631

[259] Botzanowski, T., Erb, S., Hernandez-Alba, O., Ehkirch, A., Colas, O., Wagner-Rousset, E., Rabuka, D., Beck, A., Drake, P. M., Cianferani, S., Insights from native mass 2633 spectrometry approaches for top- and middle- level characterization of site-specific antibody-drug conjugates. MAbs 2017, 862, 0-0.

[260] Beck, A., Debaene, F., Diemer, H., Wagner-Rousset, E., Colas, O., Dorsselaer, A. Van, Cianférani, S., Cutting-edge mass spectrometry characterization of originator, biosimilar and biobetter antibodies. J. Mass Spectrom. 2015, 50, 285-297.

[261] Pisupati, K., Tian, Y., Okbazghi, S., Benet, A., Ackermann, R., Ford, M., Saveliev, S., Hosfield, C. M., Urh, M., Carlson, E., Becker, C., Tolbert, T. J., Schwendeman, S. P., Ruotolo, B. T., Schwendeman, A., A Multidimensional analytical comparison of Remicade and the biosimilar Remsima. Anal. Chem. 2017, 89, 4838-4846.

[262] Ferguson, C. N., Gucinski-Ruth, A. C., Evaluation of ion mobility-mass spectrometry for comparative analysis of monoclonal antibodies. J. Am. Soc. Mass Spectrom. 2016, 27, 822-833.

[263] Tian, Y., Han, L., Buckner, A. C., Ruotolo, B. T., Collision induced unfolding of intact antibodies: rapid characterization of disulfide bonding patterns, glycosylation, and 
structures. Anal. Chem. 2015, 87, 11509-11515.

2648 [264] Pacholarz, K. J., Peters, S. J., Garlish, R. A., Henry, A. J., Taylor, R. J., Humphreys, D. 2649 P., Barran, P. E., Molecular insights into the thermal stability of mAbs with variabletemperature ion-mobility mass spectrometry. ChemBioChem 2016, 17, 46-51.

[265] Abi-Ghanem, J., Gabelica, V., Nucleic acid ion structures in the gas phase. Phys. Chem. Chem. Phys. 2014, 16, 21204-21218.

2653

[266] Hoaglund, C. S., Liu, Y., Ellington, A. D., Pagel, M., Clemmer, D. E., Gas-phase DNA: oligothymidine ion conformers. J. Am. Chem. Soc. 1997, 119, 9051-9052.

[267] Gidden, J., Ferzoco, A., Baker, E. S., Bowers, M. T., Duplex formation and the onset of helicity in poly $\mathrm{d}(\mathrm{CG}) \mathrm{n}$ oligonucleotides in a solvent-free environment. J. Am. Chem. Soc. $2004,126,15132-15140$.

[268] Gidden, J., Baker, E. S., Ferzoco, A., Bowers, M. T., Structural motifs of DNA complexes in the gas phase. Int. J. Mass Spectrom. 2005, 240, 183-193.

2660 [269] Baker, E. S., Bowers, M. T., B-DNA helix stability in a solvent-free environment. J. Am. Soc. Mass Spectrom. 2007, 18, 1188-1195.

[270] Porrini, M., Rosu, F., Rabin, C., Darré, L., Gómez, H., Orozco, M., Gabelica, V., 2663 Compaction of duplex nucleic acids upon native electrospray mass spectrometry. ACS Cent. Sci. 2017, 3, 454-461.

[271] Arcella, A., Portella, G., Ruiz, M. L., Eritja, R., Vilaseca, M., Gabelica, V., Orozco, M., Structure of triplex DNA in the gas phase. J. Am. Chem. Soc. 2012, 134, 6596-6606.

2667 [272] Baker, E. S., Bernstein, S. L., Gabelica, V., De Pauw, E., Bowers, M. T., G-quadruplexes in telomeric repeats are conserved in a solvent-free environment. Int. J. Mass Spectrom. 2006, 253, 225-237.

[273] Gabelica, V., Rosu, F., Tabarin, T., Kinet, C., Antoine, R., Broyer, M., De Pauw, E., Dugourd, P., Base-dependent electron photodetachment from negatively charged DNA strands upon 260-nm laser irradiation. J. Am. Chem. Soc. 2007, 129, 4706-4713.

2673 [274] Smargiasso, N., Rosu, F., Hsia, W., Colson, P., Baker, E. S., Bowers, M. T., De Pauw, E., Gabelica, V., G-quadruplex DNA assemblies: loop length, cation identity, and 
multimer formation. J. Am. Chem. Soc. 2008, 130, 10208-10216.

2676 [275] Rosu, F., Gabelica, V., Poncelet, H., De Pauw, E., Tetramolecular G-quadruplex formation pathways studied by electrospray mass spectrometry. Nucleic Acids Res. 2010, 38, 5217-5225.

[276] Rosu, F., Gabelica, V., Houssier, C., Colson, P., De Pauw, E., Triplex and quadruplex DNA structures studied by electrospray mass spectrometry. Rapid Commun. Mass Spectrom. 2002, 16, 1729-1736.

[277] Vairamani, M., Gross, M. L., G-quadruplex formation of thrombin-binding aptamer 2683 detected by electrospray ionization mass spectrometry. J. Am. Chem. Soc. 2003, 125, $42-43$.

[278] Balthasart, F., Plavec, J., Gabelica, V., Ammonium ion binding to DNA G-Quadruplexes: do electrospray mass spectra faithfully reflect the solution-phase species? J. Am. Soc. Mass Spectrom. 2013, 24, 1-8.

2688 [279] Rueda, M., Luque, F. J., Orozco, M., G-quadruplexes can maintain their structure in the gas phase. J. Am. Chem. Soc. 2006, 128, 3608-3619.

2690 [280] Gabelica, V., Baker, E. S., Teulade-Fichou, M. P., De Pauw, E., Bowers, M. T., Stabilization and structure of telomeric and c-myc region intramolecular Gquadruplexes: the role of central cations and small planar ligands. J. Am. Chem. Soc. 2007, 129, 895-904.

2694 [281] Kemper, P. R., Dupuis, N. F., Bowers, M. T., A new, higher resolution, ion mobility mass spectrometer. Int. J. Mass Spectrom. 2009, 287, 46-57.

[282] Shvartsburg, A. A., Tang, K., Smith, R. D., Differential ion mobility separations of peptides with resolving power exceeding 50. Anal. Chem. 2010, 82, 32-35.

[283] Shvartsburg, A. A., Tang, K., Smith, R. D., Understanding and designing field asymmetric waveform ion mobility spectrometry separations in gas mixtures. Anal.

2701 [284] Shvartsburg, A. A., Smith, R. D., Accelerated high-resolution differential ion mobility separations using hydrogen. Anal. Chem. 2011, 83, 9159-9166. 
2703 [285] Howdle, M. D., Eckers, C., Laures, A. M.-F. F., Creaser, C. S., The effect of drift gas on 2704 the separation of active pharmaceutical ingredients and impurities by ion mobility-mass 2705 spectrometry. Int. J. Mass Spectrom. 2010, 298, 72-77.

2706

[286] Fasciotti, M., Sanvido, G. B., Santos, V. G., Lalli, P. M., McCullagh, M., de Sá, G. F., 2707 Daroda, R. J., Peter, M. G., Eberlin, M. N., Separation of isomeric disaccharides by traveling wave ion mobility mass spectrometry using $\mathrm{CO} 2$ as drift gas. J. Mass

[287] Dwivedi, P., Wu, C., Matz, L. M., Clowers, B. H., Siems, W. F., Hill, H. H., Gas-phase chiral separations by ion mobility spectrometry. Anal. Chem. 2006, 78, 8200-8206.

2712

[288] Kurulugama, R. T., Darland, E., Kuhlmann, F., Stafford, G., Fjeldsted, J., Evaluation of 2713 drift gas selection in complex sample analyses using a high performance drift tube ion mobility-QTOF mass spectrometer. Analyst 2015, 140, 6834-6844.

[289] Schneider, B., Covey, T., Coy, S., Krylov, E., Nazarov, E., Control of chemical effects in the separation process of a differential mobility mass spectrometer system. Eur. J. Mass Spectrom. 2010, 16, 57.

[290] Blagojevic, V., Chramow, A., Schneider, B. B., Covey, T. R., Bohme, D. K., Differential mobility spectrometry of isomeric protonated dipeptides: modifier and field effects on ion mobility and stability. Anal. Chem. 2011, 83, 3470-3476.

[291] Varesio, E., Le Blanc, J. C. Y., Hopfgartner, G. G., Real-time 2D separation by LC × differential ion mobility hyphenated to mass spectrometry. Anal. Bioanal. Chem. 2012, 402, 2555-2564.

2724

[292] Porta, T., Varesio, E., Hopfgartner, G., Gas-phase separation of drugs and metabolites using modifier-assisted differential ion mobility spectrometry hyphenated to liquid extraction surface analysis and mass spectrometry. Anal. Chem. 2013, 85, 1177111779.

2728

[293] Chen, P.-S., Chen, S.-H., Chen, J.-H., Haung, W.-Y., Liu, H.-T., Kong, P.-H., Yang, O. H.-Y., Modifier-assisted differential mobility-tandem mass spectrometry method for detection and quantification of amphetamine-type stimulants in urine. Anal. Chim. Acta 
2016, 946, 1-8.

2732 [294] Le Masle, A., Angot, D., Gouin, C., D'Attoma, A., Ponthus, J., Quignard, A., Heinisch, S., Development of on-line comprehensive two-dimensional liquid chromatography method for the separation of biomass compounds. J. Chromatogr. A 2014, 1340, 9098.

[295] Vonk, R. J., Gargano, A. F. G., Davydova, E., Dekker, H. L., Eeltink, S., de Koning, L. J., Schoenmakers, P. J., Comprehensive two-dimensional liquid chromatography with

[296] Gargano, A. F. G., Duffin, M., Navarro, P., Schoenmakers, P. J., Reducing dilution and stationary-phase-assisted modulation coupled to high-resolution mass spectrometry applied to proteome analysis of saccharomyces cerevisiae. Anal. Chem. 2015, 87, $5387-5394$.

2744 [297] Stoll, D. R., Carr, P. W., Two-Dimensional liquid chromatography: a state of the art tutorial. Anal. Chem. 2017, 89, 519-531.

[298] Stoll, D., Danforth, J., Zhang, K., Beck, A., Characterization of therapeutic antibodies and related products by two-dimensional liquid chromatography coupled with UV absorbance and mass spectrometric detection. J. Chromatogr. B 2016, 1032, 51-60.

[299] Sorensen, M., Harmes,

D. C., Stoll, D. R., Staples, G. O., Fekete, S., Guillarme, D., Beck, A., Comparison of originator and biosimilar therapeutic monoclonal antibodies

[300] Ehkirch, A., D’Atri, V., Rouviere, F., Hernandez-Alba, O., Goyon, A., Colas, O., Sarrut, 2754 using comprehensive two-dimensional liquid chromatography coupled with time-of-flight mass spectrometry. MAbs 2016, 8, 1224-1234. M., Beck, A., Guillarme, D., Heinisch, S., Cianferani, S., An online four-dimensional HICxSEC-IMxMS methodology for in-depth characterization of antibody drug conjugates. Anal. Chem. n.d.

[301] Barcaru, A., Mol, H. G. J., Tienstra, M., Vivó-Truyols, G., Bayesian approach to peak deconvolution and library search for high resolution gas chromatography - mass 

spectrometry. Anal. Chim. Acta 2017, DOI: 10.1016/j.aca.2017.06.044.

2760 [302] Barcaru, A., Derks, E., Vivó-Truyols, G., Bayesian peak tracking: a novel probabilistic 2761 approach to match GCxGC chromatograms. Anal. Chim. Acta 2016, 940, 46-55.

2762 [303] Tolmachev, A. V., Webb, I. K., Ibrahim, Y. M., Garimella, S. V. B., Zhang, X., Anderson, 2763 G. A., Smith, R. D., Characterization of ion dynamics in structures for lossless ion 2764 manipulations. Anal. Chem. 2014, 86, 9162-9168.

2765 
2768 Figure 1: Number of papers published since 1995 in the field of IMS, IM-MS and LC-IM-MS. In 2769 this figure, the 2 years gap, mean all papers published within 2 years. Source: Scifinder scholar 2770 2017. Date of information gathering: June 2017.

2772 Figure 2: Schematic representation of a three-dimensional analysis, including a liquid 2773 chromatography separation, followed by ion mobility discrimination and time of flight mass 2774 spectrometry detection. A 3D representation of the data was also included in this 2775 representation.

2776

2777 Figure 3: Schematic representation of the commercially available IMS technology, classified as time-dispersive methods (DTIMS, TWIMS), space-dispersive methods (FAIMS, DMA) and 2779 ion trapping methods (TIMS). This figure was adapted from [2,5,18,24], with permissions from 2780 the Publishers.

2781

2782 Figure 4: Schematic representation of the three atomistic models employed for estimating gas2783 phase conformations, allowing calculation of CCS values. A: projection approximation (PA), a non-explicit method working by projecting the "shadow" of the ion onto a plane to find the average area of all the possible orientations of the molecule under investigation. B: exact hard sphere scattering (EHSS), a hard sphere collisions based method that computes the multiple

2787 scattering events without taking into account the long-range interactions. C: trajectory method 2788 (TM), an explicit model which accounts for both long-range interactions and multiple scattering events. The arrows represents the buffer gas atom trajectories.

Figure 5: (a) Calculated absolute CCS differences required to satisfy various peak-to-peak 2792 resolution conditions based on experimental CCS data for positively-charge ions based on 2793 conditional resolution model for current Agilent DTIMS instrument. Symbol key: metabolites 
2794 (filled squares), peptides (open stars), and lipids (open circles). A peak-to-peak Rs value of

27950.6 reflects conditions allowing both CCS values to be reliably determined for co-eluting 2796 isobaric species, while 1.5 represents baseline resolution. (b) Corresponding calculated 2797 percentage CCS differences for the same data. Reprinted from J. Chromatogr. A, Vol. 1416, 2798 Causon, T. J., Hann, S., Theoretical evaluation of peak capacity improvements by use of liquid 2799 chromatography combined with drift tube ion mobility-mass spectrometry., pp 47-56 (ref [82]). 2800 Copyright 2015, with permission from Elsevier.

2801

2802 Figure 6: Intensity vs. drift time for the protomers of ciprofloxacin, with the respective sites of 2803 protonation on the cyclic ketone and the piperazine moiety in the positive ionization mode. 2804 CCS values of the two protomers were also reported in this figure. This figure was adapted 2805 from [86], with permission from Waters corporation. However, a modification was applied by 2806 the authors of this application note on the original figure (Figure 5) found in [86], since one of 2807 the hypothesized protonation site was wrongly assigned in their original work.

2808

2809 Figure 7: CCSs of drug glucuronide metabolite structural isomers with different adducts and 2810 polarity. (A) Structures of drug glucuronide metabolite structural isomers. Overlays of 2811 representative arrival time distributions at $50 \mathrm{~V}$ drift voltage for (B) naringenin-4'-glucuronide 2812 (4') and naringenin-7-glucuronide (7), MW (Da) = 448.5 and for (C) $\beta$-3-estradiol-glucuronide 2813 (3) and $\beta$-17-estradiol-glucuronide (17), MW (Da) = 448.5. ATD full width half maxima and 2814 DTCCSHe averages are inset. Errors quoted are the standard deviations from at least two 2815 interday repeated measurements. Reproduced from Reading, E., Munoz-Muriedas, J., 2816 Roberts, A. D., Dear, G. J., Robinson, C. V., Beaumont, C., Anal. Chem. 2016, 88, 2273-2280 2817 (ref [112]). Copyright 2016 American Chemical Society.

2819 Figure 8: ESI(+) results from a mixture of D3-labeled hydrolyzed HEK cell lipid extracts spiked 2820 with unlabeled synthetic standards, SPH m18:1(4E)(3OH) and SPH m18:1(14E)(3OH). (A) 2821 lonograms showing separation in voltage space of the $[\mathrm{M}+\mathrm{H}]+$ ions formed from the two lipid 
2822 isomers. Each of the synthetic lipids spiked into the extract produced a peak in the black trace $2823(\mathrm{~m} / \mathrm{z} 284)$ and similar features were observed for the labeled HEK293 extract shown in the red 2824 trace $(\mathrm{m} / \mathrm{z}$ 287). (B) Ozonolysis (OzID) spectra obtained for synthetic standard (black 2825 spectrum) and the D3-labeled extract (red spectrum) corresponding to the first feature in the 2826 ionogram (i.e., $\mathrm{CV}=17 \mathrm{~V}$ ozone reaction time of $100 \mathrm{~ms}$ ). (C) OzID spectra obtained for 2827 synthetic standard (black spectrum) and the D3-labeled extract (red spectrum) corresponding 2828 to the second feature in the ionogram (i.e., $C V=20 \mathrm{~V}$ ozone reaction time of $15 \mathrm{~s}$ ). OzID 2829 product ions characteristic of the locations of the carbon-carbon double bonds are indicated 2830 by closed circles and enable assignment of the position of unsaturation to $(\Delta 14)$-position $(B)$ 2831 and ( $\Delta 4)$-position (C). Adapted from [147], with permission from the American Society for 2832 Biochemistry and Molecular Biology.

2833

2834 Figure 9: $\mathrm{HDMS}^{\mathrm{E}}$ analysis of $400 \mathrm{ng}$ Hela digest. IMS additional dimension provides an 2835 increasing number of identified peptides and proteins (38440 and 3490 respectively) with a 2836 FDR of $1 \%$ at a protein level using PLGS algorithm.

2838 Figure 10: Workflow utilized for IMS-MS analysis of membrane protein-lipid interaction by 2839 Laganowsky et al. Proteins are released from micelles by rising the source temperature and 2840 subsequently activated in the trap cell. The in-source fragmentation allows performing of MS2841 IM-MS analysis on specific ions.

2843 Figure 11: A general picture of the interaction of the full clamp loader and SSB4 before and 2844 after the addition of ssDNA. Mass spectra and the corresponding countour plots are depicted 2845 for a) $y 3 \delta \delta^{\prime} \Psi x-S S B 4$ and b) y3 $3 \delta \delta^{\prime} \Psi x-S S B 4-S S D N A_{2}$. The three dimension structure proposed

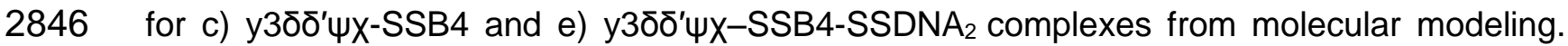
2847 Measured drift time (d) for the 36+ charge state of $y 3 \delta \delta^{\prime} \Psi x-S S B 4(b l u e)$ and $y 3 \delta \delta^{\prime} \Psi X-S S B 4-$ $2848 \mathrm{SSDNA}_{2}$ (red). A significant increase in measured collision cross section of the clamp loader2849 SS4 is observed upon DNA binding. Reprinted from J. Mol. Bio., Vol. 425, Politis, A., Park, A. 
2850 Y., Hall, Z., Ruotolo, B. T., Robinson, C. V., Integrative Modelling Coupled with Ion Mobility 2851 Mass Spectrometry Reveals Structural Features of the Clamp Loader in Complex with Single2852 Stranded DNA Binding Protein, pp 4790-4801 (ref [235]). Copyright 2013, with permission from 2853 Elsevier.

2855 Figure 12: Insights about biopharmaceutical mAbs and ADC characterization based on IM-MS 2856 data. a) Analysis of global conformation differences induced by disulfide bridge heterogeneity 2857 (Reproduced from Bagal, D., Valliere-Douglass, J. F., Balland, A., Schnier, P. D., Anal. Chem. 2858 2010, 82, 6751-6755. (ref [253]). Copyright 2010 American Chemical Society.) b) 2859 Benchmarking of different conjugation strategies by IM-MS, c) CIU fingerprint to evaluate gas2860 phase unfolding process, d) analysis of protein aggregation (oligomerization), e) 2861 characterization of the global conformation of gas-phase ions.

2862

2863 Figure 13: Separation of a mixture of 8 pharmaceutical compounds having a nominal mass of 2864316 by DMS coupled to a QqQLIT MS. DMS separation: (a) without polar modifier (nitrogen), 2865 (b) with $1.5 \%$ of $\mathrm{MeOH}$ as modifier, (c) with $1.5 \%$ of $\mathrm{EtOH}$ as modifier, and (d) with $1.5 \%$ of $\mathrm{i}$ $2866 \mathrm{PrOH}$ as modifier. With kind permission from Springer science + business media: Anal. 2867 Bioanal. Chem., Real-time 2D separation by LC $\times$ differential ion mobility hyphenated to mass 2868 spectrometry, Vol 402, 2012, 2555-2564, Varesio, E., Le Blanc, J. C. Y., Hopfgartner, G. 2869 Figure 3 (ref [291]).

2871 Figure 14: Heat map of an LC + LC-IM-qTOF measurement of a Ginkgo biloba extract (a). The separation on the second dimension column of one fraction collected between 4 and 8 min 2873 (eluting from the second dimension between 8 and $12 \mathrm{~min}$ ) from the sample is zoomed in (b). $28742 \mathrm{D}$ plot of $\mathrm{m} / \mathrm{z}$ vs. drift time extracted from 9.63 to $9.76 \mathrm{~min}$ (c). The zoomed in image shows 2875 the separation of two peaks with the same $\mathrm{m} / \mathrm{z}$ in the IM dimension (d). With kind permission 2876 from Springer science + business media: Anal. Bioanal. Chem., A novel four-dimensional 
2877 analytical approach for analysis of complex samples, Vol 408, 2016, 3751-3759, Stephan, S.,

2878 Jakob, C., Hippler, J., Schmitz, O. J. Figure 4 (ref [105]).

2879 
Dr Valentina D'Atri obtained her PhD in industrial and molecular biotechnologies at the University of Naples Federico II, Italy. She is now Postdoc at the University of Geneva, 2884 Switzerland. Her expertise includes mass spectrometry, ion mobility-mass spectrometry, 2885 molecular modelling and separation science, including HPLC, UHPLC, and HILIC. Her current 2886 research activity is in the field of biopharmaceutical proteins; in particular, her interest focuses 2887 on the analytical characterization of mAbs, ADCs, and fusion proteins by LC-MS and LC-IM2888 MS.

Dr Tim Causon obtained his PhD at the University of Tasmania, Australia. He is now Senior 2891 Scientist in the group of Instrumental Analytical Chemistry and Metabolomics at the 2892 Department of Chemistry of the University of Natural Resources and Life Sciences in Austria. 2893 His research interests centre on mass spectrometry, ion mobility-mass spectrometry and 2894 separation science, including analytical method development for metabolomics, as well as 2895 fundamental analytical studies.

Dr. Oscar Hernandez Alba has joined the BioOrganic Mass Spectrometry Laboratory (LSMBO) at the Hubert Curien Pluridisciplinary Institute (IPHC, University of Strasbourg, 2899 CNRS UMR7178) in Strasbourg as a postdoctoral researcher. He obtained his PhD degree at 2900 the University of Paris-Sud. His thesis was focused on developing a multimodal approach 2901 based on a single mass spectrometer, which incorporates ion mobility spectrometry and 2902 infrared spectroscopy in order to characterize the structure of mobility- and mass-selected 2903 molecular ions. He is currently working on the characterization of biopharmaceuticals and 2904 noncovalent protein/protein complex using native mass spectrometry and ion mobility 2905 spectrometry. 
2907 Aline Mutabazi graduated from the School of Pharmaceutical Sciences of the University of 2908 Geneva as a pharmacist in 2016. After a master thesis about the development and validation 2909 of a LC-MS method for the analysis of neonicotinoids in honey, she joined Jean-Luc Veuthey 2910 and Davy Guillarme's team in January 2017 to begin a Ph.D. thesis focused on ion mobility 2911 applied to the analysis of pharmaceutical compounds.

2912

2913 Prof. Jean-Luc Veuthey is professor at the School of Pharmaceutical Sciences, University of 2914 Geneva, Switzerland. He also acted as President of the School of Pharmaceutical Sciences, 2915 Vice-Dean of the Faculty of Sciences and finally Vice-Rector of the University of Geneva. His 2916 research domains are: development of separation techniques in pharmaceutical sciences, and 2917 more precisely: study of the impact of sample preparation procedures in the analytical 2918 process; fundamental studies in liquid and supercritical chromatography; separation 2919 techniques coupled with mass spectrometry; analysis of drugs and drugs of abuse in different 2920 matrices. He published more than 300 articles in peer-review journals.

Dr. Sarah Cianférani holds a PhD degree in analytical chemistry from the University of 2923 Strasbourg, France. She is now a CNRS research director and heads the BioOrganic Mass 2924 Spectrometry Laboratory (LSMBO) at the Hubert Curien Pluridisciplinary Institute (IPHC, 2925 University of Strasbourg, CNRS UMR7178) in Strasbourg. Her expertise focused on 2926 developments in structural mass spectrometry (native MS, ion mobility, H/D exchange and 2927 crosslinking) and applications of advanced native mass spectrometry and native ion-mobility 2928 mass spectrometry methodologies for biological noncovalent complex characterization, and especially biopharmaceuticals (mAb-related compounds). She is a co-author of more than 110 scientific papers related to mass spectrometry analysis of proteins.

2932 Dr Davy Guillarme holds a PhD degree in analytical chemistry from the University of Lyon, 2933 France. He is now senior lecturer at the University of Geneva in Switzerland. He authored 190 2934 journal articles related to pharmaceutical analysis. His expertise includes HPLC, UHPLC, 
2935 HILIC, LC-MS, SFC, analysis of proteins and mAbs. He is an editorial advisory board member 2936 of several journals including Journal of Chromatography A, Journal of Separation Science, LC2937 GC North America and others...

2938 